\title{
Matrix product states and equivariant topological field theories for bosonic symmetry-protected topological phases in $(1+1)$ dimensions
}

\author{
Ken Shiozaki ${ }^{a}$ and Shinsei $\mathbf{R y u}^{b}$ \\ ${ }^{a}$ Department of Physics, University of Illinois at Urbana Champaign, \\ 1110 West Green Street, Urbana, IL 61801, U.S.A. \\ ${ }^{b}$ James Franck Institute and Kadanoff Center for Theoretical Physics, University of Chicago, \\ 5640 South Ellis Ave, Chicago, IL 60637, U.S.A. \\ E-mail: ken.shiozaki@riken.jp, ryuu@uchicago.edu
}

AbSTRaCT: Matrix Product States (MPSs) provide a powerful framework to study and classify gapped quantum phases - symmetry-protected topological (SPT) phases in particular - defined in one dimensional lattices. On the other hand, it is natural to expect that gapped quantum phases in the limit of zero correlation length are described by topological quantum field theories (TFTs or TQFTs). In this paper, for $(1+1)$-dimensional bosonic SPT phases protected by symmetry $G$, we bridge their descriptions in terms of MPSs, and those in terms of $G$-equivariant TFTs. In particular, for various topological invariants (SPT invariants) constructed previously using MPSs, we provide derivations from the point of view of $(1+1)$ TFTs. We also discuss the connection between boundary degrees of freedom, which appear when one introduces a physical boundary in SPT phases, and "open" TFTs, which are TFTs defined on spacetimes with boundaries.

KEYwords: Topological Field Theories, Topological States of Matter, Global Symmetries, Lattice Quantum Field Theory

ARXiv EPrint: 1607.06504 


\section{Contents}

1 Introduction $\quad 2$

2 Classification and topological invariants of SPT phases using MPSs 3

2.1 Symmetry and group cohomology classification 3

2.2 Edge degrees of freedom 4

2.3 Simple and fixed point MPSs 5

2.4 Topological invariants $\quad 7$

2.4.1 Topological invariants in terms of ground state wave functions 8

3 G-equivariant topological field theories and MPSs $\quad 16$

$\begin{array}{lll}3.1 & \text { Some basics of TFTs } & 17\end{array}$

$\begin{array}{lll}3.2 & G \text {-equivariant oriented closed TFTs } & 18\end{array}$

$\begin{array}{lll}\text { 3.2.1 General solution for semi simple cases } & 21\end{array}$

$3.3 \quad G$-equivariant unoriented closed TFTs 22

3.3.1 General solution for simple cases $\quad 24$

3.3.2 Relation to MPS 24

$\begin{array}{ll}3.4 & G \text {-equivariant open and closed TFTs }\end{array}$

$\begin{array}{lll}3.4 .1 & \text { Relation to open MPS } & 27\end{array}$

$\begin{array}{lll}3.4 .2 & \text { Equivariant Cardy conditions and boundary states } & 28\end{array}$

$\begin{array}{lll}3.4 .3 & \text { Crosscap invariant in open chain } & 30\end{array}$

3.5 State sum construction 31

3.5.1 Fukuma-Hosono-Kawai state sum construction 31

$\begin{array}{lll}\text { 3.5.2 } & G \text {-equivariant state sum construction } & 32\end{array}$

3.5.3 Partition functions 33

$\begin{array}{lll}3.5 .4 & \text { Cobordisms } & 34\end{array}$

4 Conclusion $\quad 36$

$\begin{array}{ll}\text { A Group cohomology } & 38\end{array}$

$\begin{array}{ll}\text { B Projective representation } & 39\end{array}$

C Orbifolding: Dijkgraaf-Witten theory in $(1+1) d \quad 40$

D Algebraic relations in equivariant open and closed TFTs 40

D.1 Closed TFT 40

D.2 Open TFT 42 


\section{Introduction}

Symmetry-protected topological (SPT) phases of matter are gapped phases of quantum many-body systems with short-range entanglement. They are topologically distinct from topologically trivial states in the presence of symmetries. In other words, SPT phases are separated from topologically trivial phases by quantum critical points. Here, "shortrange entanglement" means in particular the absence of topological order, and hence the uniqueness of the ground state even when the system is put on an arbitrary spatial manifold. (This property is often called "invertible", and hence SPT phases are said to have an invertible topological order.)

Bosonic SPT phases in $(1+1)$ dimensions are known to be classified by the second group cohomology $H^{2}(G, \mathrm{U}(1))$. [1] For quantum many-body systems defined on one-dimensional lattices, this can be most easily seen from the matrix product state (MPS) representations of the quantum ground states of SPT phases. [2-5]

On the other hand, deep inside a gapped phase where the correlation length is very short (order of a few lattice constant), one could expect that the universal properties of the system can be described in terms of a topological quantum field theory (TQFT or TFT). The canonical examples include Chern-Simons theories, which describe various fractional quantum Hall liquids, and the BF theory, which describes the topological limit of the $\mathbb{Z}_{n}$ lattice gauge theory. $[6,7]$

In this paper, we will undertake the task of bridging the descriptions of $(1+1) \mathrm{d}$ bosonic SPT phases using MPSs, and those using $(1+1) d$ TFTs. For $(1+1) d$ bosonic SPT phases protected by symmetry $G$, where $G$ is a symmetry group, the relevant TFTs are $G$ equivariant TFTs discussed by Turaev and Moore-Segal. [8-12] We in particular address the following two issues.

The first issue is about topological invariants (SPT invariants) of $(1+1) \mathrm{d}$ bosonic SPT phases. These are quantities (numbers) which one can compute for a given quantum ground state of a gapped $(1+1) d$ system, and take the same value anywhere in a given gapped phase. I.e., they are stable and remain unchanged against adiabatic deformations of Hamiltonians so far as one stays within a given gapped phase. On the one hand, several topological invariants for bosonic SPT phases have been constructed so far by using MPSs. [5] In this paper, we will rederive these invariants by using $G$-equivariant TFTs in $(1+1)$ d. The topological invariants are nothing but the partition functions of TFTs.

Second, the hallmark of $(1+1)$ d SPT phases is the presence of boundary degrees of freedom that appear when the SPT phases are terminated by boundaries. The canonical example is the spin $1 / 2$ that appears at the end of the spin 1 Haldane spin chain. In terms of MPSs, these physical boundary degrees of freedom are captured by degrees of freedom living in the auxiliary bond (entanglement) Hilbert space. On the TFT side, a natural framework to discuss the boundary degrees of freedom is an "open" TFT. [11, 13] Open TFTs are TFTs defined on the $(1+1)$ d spacetime which has $(1+0)$ d boundaries. In this paper, we will make an attempt to make a dictionary between MPSs with boundaries and open TFTs.

The rest of the paper is organized as follows: in section 2, we introduce the descriptions of bosnic SPT phases in $(1+1) d$ using MPSs. In particular, we review the known construction of various topological invariants for $(1+1) \mathrm{d}$ SPT phases built out of MPSs. 
By using the fixed point MPSs, we confirm that these topological invariants characterizes the elements of the group cohomology $H^{2}(G, \mathrm{U}(1))$, and describe the procedure to extract these from a given quantum ground state. (All results in section 2 are known in the literature, so readers who are familiar with the MPS descriptions of $(1+1)$ d bosonic SPT phases and their topological invariants can skip this section.)

In section 3, we introduce G-equivariant TFTs following Moore and Segal. [11] We discuss both closed and open TFTs; in closed TFTs we consider the $(1+1) d$ spacetime which has no boundary, whereas in open TFTs the $(1+1) d$ spacetime has $(1+0) d$ boundaries.

In section 3.5, we will derive the topological invariants from the point of view of $(1+1) \mathrm{d}$ TFTs. To this end, we evaluate the partition functions of $(1+1) d$ TFTs by using the socalled state sum construction [14]. Introducing an orientation reversing operation on Frobenius algebras enables us to define partition function on the real projective plane $\mathbb{R} P^{2}[15]$.

Finally, appendices are devoted to an introduction to the group cohomology, and projective representations, the relation to orbifolded theories $((1+1)$ d Dijkgraaf-Witten theories), and the derivations of algebraic relations in $G$-equivariant open and closed TFTs.

\section{Classification and topological invariants of SPT phases using MPSs}

In this section, we briefly review the topological classification of bosonic SPT phases in $(1+1) \mathrm{d}[1,3,4]$, and their topological invariants [5]. From the field theoretical point of view, bosonic SPT phases are described by $G$-equivariant TFTs [11], which will be introduced in the next section.

\subsection{Symmetry and group cohomology classification}

Let us consider a short range entangled pure state $|\Psi\rangle$ on a closed chain of length $L$, which is represented by a MPS

$$
|\Psi\rangle=\sum_{\left\{m_{i}\right\}} \operatorname{Tr}\left(A_{m_{1}} \cdots A_{m_{L}}\right)\left|m_{1} \cdots m_{L}\right\rangle, \quad\left|m_{1} \cdots m_{L}\right\rangle=\left|m_{1}\right\rangle_{1} \otimes \cdots \otimes\left|m_{L}\right\rangle_{L},
$$

where $|m\rangle_{j}$ represents a state in the physical Hilbert space at the $j$-th site and $A_{m_{j}}$ is a $\chi \times \chi$ matrix which acts on the auxiliary Hilbert space or the entanglement Hilbert space living on the bonds; the trace is taken over the $\chi \times \chi$ dimensional auxiliary Hilbert space. Here and henceforth, we assume the translational symmetry for simplicity.

Let $G$ be a symmetry group. The symmetry group $G$ possibly includes orientation reversing symmetries (time-reversal or inversion symmetry, say). For the purpose of specifying the orientation reversing symmetries, let us introduce a homomorphism $\phi: G \rightarrow G_{0}$, where $G_{0}$ is a group consisting of the orientation preserving symmetries. The symmetry action $g \in G$ is defined on the basis $|m\rangle_{j}$ by a linear representation of $G$,

$$
\begin{aligned}
& \hat{g}\left(|m\rangle_{j}\right)=|n\rangle_{j}\left[U_{g}\right]_{n m}, \quad U_{g} U_{h}=U_{g h}, \quad(g \text { is on-site unitary symmetry }), \\
& \hat{T}\left(|m\rangle_{j}\right)=|n\rangle_{j}\left[U_{T}\right]_{n m}, \quad U_{T} U_{g}^{*}=U_{T g}, \quad(T \text { is time-reversal symmetry }), \\
& \hat{P}\left(|m\rangle_{j}\right)=|n\rangle_{L-j}\left[U_{P}\right]_{n m}, \quad U_{P} U_{g}=U_{P g}, \quad(P \text { is inversion symmetry }),
\end{aligned}
$$

for any $h \in G$. 
[a]

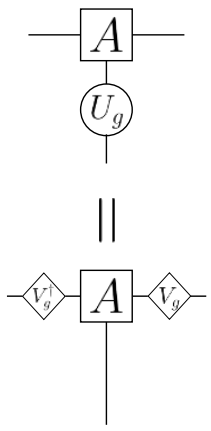

[b]

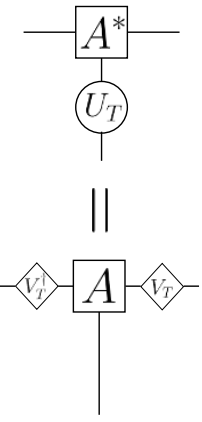

[c]

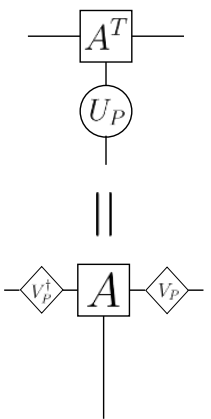

Figure 1. The symmetry property of $A_{m}$ under [a] on-site unitary, [b] time-reversal, and [c] inversion symmetries, respectively.

By choosing different $A$, one can construct the ground states of gapped phases in $(1+1)$ dimensions. One can consider to classify these gapped phase topologically, in the presence of a prescribed symmetry $G$. The topological classification of $(1+1)$ d SPT phases of bosons is given by the classification of the symmetry action on $A_{m}$. Under the assumption that $|\Psi\rangle$ is a pure state, one can show [16]

$$
\begin{array}{rlrl}
{\left[U_{g}\right]_{m n} A_{n}} & =e^{i \theta_{g}} V_{g}^{\dagger} A_{m} V_{g}, & & (g \text { is on-site unitary symmetry }), \\
{\left[U_{T}\right]_{m n} A_{n}^{*}=e^{i \theta_{T}} V_{T}^{\dagger} A_{m} V_{T},} & & (T \text { is time-reversal symmetry }), \\
{\left[U_{P}\right]_{m n} A_{n}^{T}=e^{i \theta_{P}} V_{P}^{\dagger} A_{m} V_{P},} & & (P \text { is inversion symmetry }),
\end{array}
$$

where $e^{i \theta_{g}}, e^{i \theta_{T}}$, and $e^{i \theta_{P}}$ are 1-dimensional linear representations of $G$, and $V_{g}, V_{T}$, and $V_{P}$ act on the entanglement Hilbert space, and obey, for any $h \in G$,

$$
\left\{\begin{array}{l}
V_{g} V_{h}=b(g, h) V_{g h} \quad(g \text { is on-site unitary symmetry }) \\
\left.V_{g} V_{h}^{*}=b(g, h) V_{g h} \text { ( } g \text { is time-reversal or inversion symmetry }\right)
\end{array}\right.
$$

with a $\mathrm{U}(1)$ phase $b(g, h) \in \mathrm{U}(1)$ (2-cocycle). These symmetry actions on $A_{m}$ are diagrammatically represented in figure 1 . (In the figure, we neglect the 1-dimensional representation $e^{i \theta_{g}}$.) From the associativity condition of $V_{g}$, it follows that $b(g, h)$ is a representative of $\phi$-twisted second group cohomology $H^{2}\left(G, \mathrm{U}(1)_{\phi}\right)$. (Here, " $\phi$-twisted" means the $g \notin G_{0}$ action on $\mathrm{U}(1)$ group is defined by complex conjugate. See appendix A.) The factor group $[b(g, h)] \in H^{2}\left(G, \mathrm{U}(1)_{\phi}\right)$ classifies how symmetry $G$ acts on the short-range entangled pure state $|\Psi\rangle$ on the 1-dimensional closed chain.

\subsection{Edge degrees of freedom}

In a non-trivial SPT phase (specified by a nontrivial group cohomology $[b(g, h)] \in$ $\left.H^{2}\left(G, \mathrm{U}(1)_{\phi}\right),[b(g, h)] \neq 0\right)$ on an open chain $I=\{1, \ldots, L\}$, there emerge edge degrees of freedom where the symmetry action of $G$ is "fractionalized". The boundary degrees of 
freedom can be discussed by using the MPS of the open chain:

$$
\Psi\left(\left\langle v_{L}|\otimes| v_{R}\right\rangle\right)=\sum_{\left\{m_{i}\right\}} v_{L}^{\dagger} A_{m_{1}} \cdots A_{m_{L}} v_{R}\left|m_{1} \cdots m_{L}\right\rangle, \quad\left\langle v_{L}\left|\in V^{*}, \quad\right| v_{R}\right\rangle \in V,
$$

where $\left\langle v_{L}\right|$ and $\left|v_{R}\right\rangle$ specify boundary conditions and belong to the edge Hilbert space $V^{*}$ and $V$, respectively. $V$ is a $b(g, h)$-projective representation defined by $(2.8)$ and $V^{*}$ is the its conjugate representation. Here, the symmetry fractionalization is realized in the following sense: a symmetry action $g \in G_{0}$ on the MPS on the open chain is given by

$$
\hat{g} \Psi\left(\left\langle v_{L}|\otimes| v_{R}\right\rangle\right)=\sum_{\left\{m_{i}\right\}} v_{L}^{\dagger} V_{g}^{\dagger} A_{m_{1}} \cdots A_{m_{L}} V_{g} v_{R}\left|m_{1} \cdots m_{L}\right\rangle=\Psi\left(\left\langle v_{L}\left|V_{g}^{\dagger} \otimes V_{g}\right| v_{R}\right\rangle\right),
$$

where a symmetry operation $g \in G_{0}$ is projectively represented at the edges, as opposed to the $g$ action on the bulk physical degrees of freedom, which is a linear representation.

For an on-site unitary symmetry $g \in G_{0}$, we can introduce a $g$-twisted MPS

$$
\left|\Psi_{g}\right\rangle:=\sum_{\left\{m_{i}\right\}} \operatorname{Tr}\left(A_{m_{1}} \cdots A_{m_{L}} V_{g}\right)\left|m_{1} \cdots m_{L}\right\rangle, \quad\left(g \in G_{0}\right) .
$$

From the perspective of Hamiltonians, $\left|\Psi_{g}\right\rangle$ is a ground state of a Hamiltonian with a $g \in G_{0}$ symmetry defect.

For our purpose to make a comparison between MPSs and TFTs, it is useful to introduce an open to closed map $\imath^{g}$ and a closed to open map $\imath_{g}[11]$ as

$$
\begin{aligned}
\imath^{g}\left(\Psi\left(\left\langle v_{L}|\otimes| v_{R}\right\rangle\right)\right) & :=\left\langle v_{L}\left|V_{g}^{\dagger}\right| v_{R}\right\rangle\left|\Psi_{g}\right\rangle, & & \left(g \in G_{0}\right), \\
\imath_{g}\left(\left|\Psi_{g}\right\rangle\right) & :=\sum_{a} \Psi\left(\left\langle a\left|\otimes V_{g}\right| a\right\rangle\right), & & \left(g \in G_{0}\right) .
\end{aligned}
$$

Here $\{|a\rangle\}_{a=1}^{\operatorname{dim} V}$ is a basis of $V$.

Finally, we also introduce a formal "gluing" operation of two open MPSs by

$$
\Psi\left(\left\langle v_{L}|\otimes| v_{R}\right\rangle\right) \cdot \Psi\left(\left\langle w_{L}|\otimes| w_{R}\right\rangle\right):=\left\langle w_{L} \mid v_{R}\right\rangle \Psi\left(\left\langle v_{L}|\otimes| w_{R}\right\rangle\right) .
$$

This will be also useful when we make a comparison between MPSs and TFTs.

\subsection{Simple and fixed point MPSs}

To study ground states deep inside a gapped phase, or to study SPT phases in general, it is useful and convenient to introduce a simple and fixed point MPS. "Simple" here means that the transfer matrix $T_{a b, c d}=\sum_{m}\left[A_{m}\right]_{a b}\left[A_{m}^{*}\right]_{c d}$ has an only one eigenstate with unit magnitude of eigenvalue $|\nu|=1$, i.e., unique ground state [17]. "Fixed point" means that we are in the limit of zero correlation length. It is in this limit where we expect SPT phases and the corresponding MPSs are faithfully described by TQFTs. In the following, we will construct fixed point MPSs $|\Psi\rangle$ with a nontrivial group cohomology $H^{2}\left(G, \mathrm{U}(1)_{\phi}\right)$.

Let $G$ be a symmetry group with nontrivial group cohomology $H^{2}\left(G, \mathrm{U}(1)_{\phi}\right) \neq 0$. We fix a nontrivial 2-cocycle $b(g, h) \in Z^{2}\left(G, \mathrm{U}(1)_{\phi}\right),[b(g, h)] \neq 0$. We choose two $b(g, h)$ projective representations $V$ which satisfy (2.8). We use the tensor product representation 
[a]

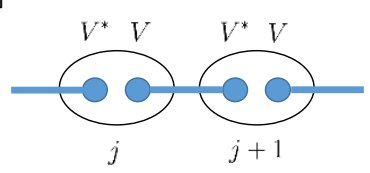

[b]

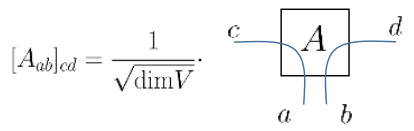

[c]

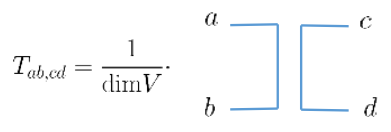

Figure 2. [a] A fixed point MPS. The blue bond represents the singlet representation in $V^{*} \otimes V$. [b] $A$ matrix. [c] The transfer matrix.

$V^{*} \otimes V$ as a physical Hilbert space, where $V^{*}$ is the complex representation of $V$. Note that in the product representation, the effect of the 2-cocycle cancels, $b^{*}(g, h) b(g, h)=1$. For each site $j$, the basis is given by $\left\{|a\rangle_{j}^{L} \otimes|b\rangle_{j}^{R}\right\}$, where $|a\rangle_{j}^{L}\left(|b\rangle_{j}^{R}\right)$ is the basis of $V^{*}(V)$ and transformed as

$$
\begin{aligned}
& \begin{cases}\hat{g}\left(|a\rangle_{j}^{L} \otimes|b\rangle_{j}^{R}\right)=|c\rangle_{j}^{L} \otimes|d\rangle_{j}^{R}\left[V_{g}^{*}\right]_{c a}\left[V_{g}\right]_{d b}, & (g \text { is on-site unitary symmetry }), \\
{\left[U_{g}\right]_{a b, c d}=\left[V_{g}^{*}\right]_{a c}\left[V_{g}\right]_{b d},}\end{cases} \\
& \left\{\begin{array}{l}
\hat{T}\left(|a\rangle_{j}^{L} \otimes|b\rangle_{j}^{R}\right)=|c\rangle_{j}^{L} \otimes|d\rangle_{j}^{R}\left[V_{T}^{*}\right]_{c a}\left[V_{T}\right]_{d b}, \hat{T} i \hat{T}^{-1}=-i, \\
{\left[U_{T}\right]_{a b, c d}=\left[V_{T}^{*}\right]_{a c}\left[V_{T}\right]_{b d},}
\end{array}\right. \\
& \left\{\begin{array}{l}
\hat{P}\left(|a\rangle_{j}^{L} \otimes|b\rangle_{j}^{R}\right)=|c\rangle_{L-j}^{L} \otimes|d\rangle_{L-j}^{R}\left[V_{P}^{*}\right]_{c b}\left[V_{P}\right]_{d a}, \\
{\left[U_{P}\right]_{a b, c d}=\left[V_{P}^{*}\right]_{a d}\left[V_{P}\right]_{b c},}
\end{array}\right.
\end{aligned}
$$

Note that $V$ and $V^{*}$ representations are exchanged under the inversion transformation.

To write down ground state wave functions, we make use of a singlet included in the decomposition of the product representations, $V^{*} \otimes V=1 \oplus \cdots$. The fixed point MPS can be constructed as the product state of singlet bonds (figure 2 [a]) as

$$
|\Psi\rangle=\cdots \otimes\left(\sum_{a}|a\rangle_{j}^{R} \otimes|a\rangle_{j+1}^{L}\right) \otimes\left(\sum_{b}|b\rangle_{j+1}^{R} \otimes|b\rangle_{j+2}^{L}\right) \otimes \cdots .
$$

This can be written in the MPS form as

$$
|\Psi\rangle=\sum \cdots\left[A_{b_{j} c_{j}}\right]_{a_{j} a_{j+1}}\left[A_{b_{j+1} c_{j+1}}\right]_{a_{j+1} a_{j+2}} \cdots\left|\cdots\left(b_{j} c_{j}\right)\left(b_{j+1} c_{j+1}\right) \cdots\right\rangle
$$

with

$$
\left[A_{a b}\right]_{c d}=\frac{1}{\sqrt{\operatorname{dim} V}} \delta_{a c} \delta_{b d}
$$

(See figure $2[\mathrm{~b}]$.) Here $(a b)$ is the physical index whereas $(c d)$ is the entanglement index. We abbreviated $\left|b_{j}\right\rangle_{j}^{L} \otimes\left|c_{j}\right\rangle_{j}^{R}$ by $\left|\left(b_{j} c_{j}\right)\right\rangle$. The prefactor $\frac{1}{\sqrt{\operatorname{dim} V}}$ is the normalization constant. The MPS $|\Psi\rangle$ is the AKLT state [18] without any projection on the site degrees of freedom. [19] 
For the fixed point MPSs, the transfer matrix $T_{a b, a^{\prime} b^{\prime}}$ (figure $2[\mathrm{c}]$ ) is given by

$$
T_{a b, c d}=\sum_{e f}\left[A_{e f}\right]_{a b}\left[A_{e f}^{*}\right]_{c d}=\frac{1}{\operatorname{dim} V} \delta_{a c} \delta_{b d} .
$$

In any symmetries, we have the following group action on $\left[A_{a b}\right]_{c d}$ for the fixed point MPS,

$$
\begin{aligned}
{\left[U_{g}\right]_{a b, e f}\left[A_{e f}\right]_{c d}=\frac{1}{\sqrt{\operatorname{dim} V}}\left[V_{g}^{*}\right]_{a c}\left[V_{g}\right]_{b d}, \quad(g \text { is on-site unitary symmetry }), } \\
{\left[U_{T}\right]_{a b, e f}\left[A_{e f}^{*}\right]_{c d}=\frac{1}{\sqrt{\operatorname{dim} V}}\left[V_{T}^{*}\right]_{a c}\left[V_{T}\right]_{b d}, \quad(T \text { is time-reversal symmetry }), } \\
{\left[U_{P}\right]_{a b, e f}\left[A_{e f}^{T}\right]_{c d}=\frac{1}{\sqrt{\operatorname{dim} V}}\left[V_{P}^{*}\right]_{a c}\left[V_{P}\right]_{b d}, \quad(P \text { is inversion symmetry }) . }
\end{aligned}
$$

Finally, it is worth pointing out that in fixed point MPSs the length of the MPS chain is irrelevant because of the zero correlation length. I.e., since they are at a renormalization group fixed point, increasing/decreasing the number of cites does not change the essential properties of the state. For this reason, we always identify MPS chains with different lengths as

$$
\operatorname{Tr}\left(A_{m_{1}} \cdots A_{m_{L}}\right)\left|m_{1} \cdots m_{L}\right\rangle \sim \operatorname{Tr}\left(A_{m_{1}} \cdots A_{m_{L}} A_{m_{L+1}}\right)\left|m_{1} \cdots m_{L} m_{L+1}\right\rangle .
$$

\subsection{Topological invariants}

In this section, we will construct and discuss topological invariants of bosonic SPT phases in $(1+1) d$ using the MPS.

We start by listing topological invariants. Detailed descriptions of topological invariants will follow shortly. There are three types of topological invariants, which are defined in terms of the data of 2-cocycle $\{b(g, h)\}$ :

- The discrete torsion phase (partition function on $T^{2}$ with twist)

$$
\epsilon(g, h)=\frac{b(g, h)}{b(h, g)}, \quad V_{g} V_{h}=\epsilon(g, h) V_{h} V_{g}, \quad g, h \in G_{0}, \quad g h=h g .
$$

- The crosscap invariant (partition function on $\mathbb{R} P^{2}$ )

$$
\theta(g):=b(g, g), \quad V_{g} V_{g}^{*}=\theta(g), \quad g \notin G_{0}, \quad g^{2}=1 .
$$

- The Klein bottle invariant (partition function on the Klein bottle with twist)

$$
\kappa(g ; h)=\frac{b\left(g, h^{-1}\right) b\left(h, h^{-1}\right)}{b(h, g)}, \quad V_{g} V_{h}^{T}=\kappa(g ; h) V_{h} V_{g}, \quad g \notin G_{0}, h \in G_{0}, \quad g h^{-1}=h g .
$$

Several comments are in order.

- First, in (2.26), (2.27) and (2.28), and throughout this subsection, we omit the 1dimensional representation $\left\{e^{i \theta_{g}}\right\}$ for simplicity. 
- One can check easily that these quantities are left unchanged under the 1-coboundary $b(g, h) \mapsto b(g, h) a_{g} a_{g}^{\phi(g)} a_{g h}^{-1}, a_{g} \in \mathrm{U}(1)$.

- One can give interpretations to these topological invariants in terms of spacetime path integrals. We will mention these interpretations later in this subsection, and also in section 3-3.5 from the TFT point of view. In short, these three topological invariants are interpreted as the partition function on the torus, the projective plane, and the Klein bottle respectively. For this reason, we will often refer the topological invariants as the partition functions (on the torus, the projective plane, and the Klein bottle).

- Finally, the above three SPT invariants are not independent. One can show

$$
\begin{aligned}
\epsilon(g, 1) & =\epsilon(1, g)=1 & & \text { for } g \in G_{0}, \\
\epsilon(h, g) & =\epsilon(g, h)^{-1} & & \text { for } g, h \in G_{0}, g h=h g, \\
\epsilon(g, h k) & =\epsilon(g, h) \epsilon(g, k) & & \text { for } g, h, k \in G_{0}, g h=h g, g k=k g, \\
\kappa(g ; 1) & =1 & & \text { for } g \notin G_{0}, \\
\kappa(g ; h k) & =\kappa(g ; h) \kappa(g ; k) & & \text { for } g \notin G_{0}, h, k \in G_{0}, g h^{-1}=h g, g k^{-1}=k g, \\
\kappa(k g ; h) & =\kappa\left(g k^{-1} ; h\right)=\epsilon(k, h) \kappa(g ; h) & & \text { for } g \notin G_{0}, h, k \in G_{0}, g h^{-1}=h g, h k=k h, \\
\theta(h g) & =\theta\left(g h^{-1}\right)=\kappa(g ; h) \theta(g) & & \text { for } g \notin G_{0}, h \in G_{0}, g^{2}=1, g h^{-1}=h g .
\end{aligned}
$$

In many cases, the Klein bottle SPT invariant $\kappa(g ; h)$ can be written in terms of $\epsilon(g, h)$ and $\theta(g)$. This is however not always the case. A simple example in which the Klein bottle invariant does not reduce to the other invariants is an SPT phase protected by $G=\mathbb{Z}_{4}=\left\{1, \sigma, \sigma^{2}, \sigma^{3}\right\}$. Here, the generator $\sigma$ is inversion/timereversal. For a nontrivial projective representation generated by $V_{\sigma}=e^{-i s_{y} \frac{\pi}{4}}$, where $s_{y}=\left(\begin{array}{cc}0 & -i \\ i & 0\end{array}\right)$ is the $y$-component of the Pauli matrix, from $V_{\sigma} V_{\sigma^{2}}^{T}=-V_{\sigma^{2}} V_{\sigma}$, the Klein bottle SPT invariant reads $\kappa\left(\sigma ; \sigma^{2}\right)=-1$.

\subsubsection{Topological invariants in terms of ground state wave functions}

As mentioned, the topological invariants can be interpreted by using the path integral formalism. In the rest of this subsection, we will instead use the operator formalism, and in particular aim to extract the topological invariant solely by using ground state wave functions. (Apart from our goal of bridging MPSs and TFTs, expressing topological invariants solely in terms of ground state wave functions may have practical (numerical) merits.)

In order to discuss and define these topological invariants, one important ingredient is gauging symmetry. Here, by gauging, we mean coupling the system to the background flat $G$-bundle. We will describe how this can be done within MPSs for on-site unitary symmetry in section 2.4.1. The same gauging procedure can be introduced by using the path-integral. (In addition, one could promote the background gauge field into a dynamical one. This procedure is often called orbifolding to distinguish it from gauging. In this paper, for the purpose of describing SPT phses, we will consider gauging but not orbifolding. Orbifolding leads to the so-called Dijkgraaf-Wittten theories, which we briefly discuss in appendix C.) 

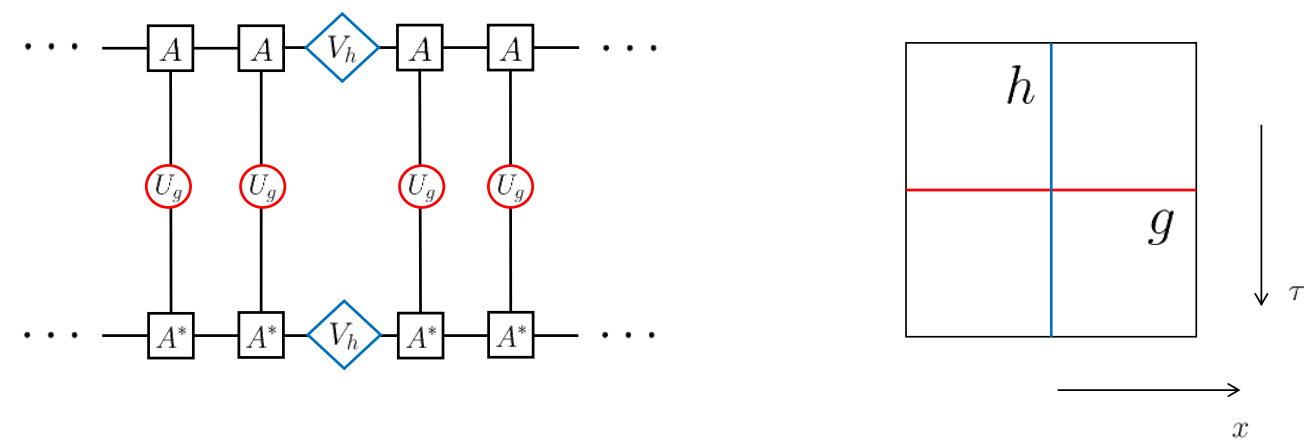

Figure 3. [a] MPS expression of the symmetry action on the twisted ground state. [b] The equivalent path integral on the 2-torus $T^{2}$. The blue and red lines express the symmetry defect lines.

As for the crosscap invariant, we need to introduce a "trick" within the operator formalism in order to mimic the effect of putting the theory on $\mathbb{R} P^{2}$ in the path integral formalism. This can be done in two different ways, depending on whether the symmetry group includes spatial inversion or time-reversal. Following Pollmann and Turner [5] we will introduce two operations, "partial inversion" and "adjacent partial transposition", for spatial inversion and time-reversal, respectively. When interpreted in the path integral formalism, these operations effectively create $\mathbb{R} P^{2}$ as the spacetime manifold.

Introducing such a partial space-time twist operator is a useful way to detect SPT topological invariant which cannot be represented by the partition function on a mapping torus. ${ }^{1}$ This prescription can also be applied to fermionic SPT invariants [20] and SPT phases in more general space dimensions. [21]

For all topological invariants, the fact that they can be extracted from ground state wave functions can be easily proven if we use the fixed point MPS.

Discrete torsion phase (1): symmetry action on twisted ground state. Let us first express the torus topological invariant (2.26) by using ground state wave functions.

From the MPS and the projective representation $\left\{V_{g}\right\}_{g \in G}$, we can construct the MPS $\left|\Psi_{h}\right\rangle$ with boundary condition twisted by an on-site unitary symmetry $h \in G_{0}$ as

$$
\left|\Psi_{h}\right\rangle=\sum_{\left\{m_{i}\right\}} \operatorname{Tr}\left(A_{m_{1}} \cdots A_{m_{L}} V_{h}\right)\left|m_{1} \cdots m_{L}\right\rangle .
$$

Then, for a global unitary symmetry, $g \in G_{0}$,

$$
\hat{g}\left|\Psi_{h}\right\rangle=\sum_{\left\{m_{i}\right\}} \operatorname{Tr}\left(A_{m_{1}} \cdots A_{m_{L}} V_{g} V_{h} V_{g}^{-1}\right)\left|m_{1} \cdots m_{L}\right\rangle=\frac{b(g, h)}{b\left(g h g^{-1}, g\right)}\left|\Psi_{g h g^{-1}}\right\rangle .
$$

\footnotetext{
${ }^{1}$ A mapping torus is space-time manifold which takes the form $M \times_{f} S^{1}:=M \times[0,1] /\{(x, 0) \sim(f(x), 1)\}$, where $f: M \rightarrow M$ is a diffeomorphism. In (invertible) TFTs, the partition function $Z\left(M \times{ }_{f} S^{1}\right)$ on $M \times_{f} S^{1}$ is given by the expectation value of operator $\hat{f}$ representing diffeomorphism $f$ on the ground state wave function $\left|\Psi_{M}\right\rangle$ on $M$ as $Z\left(M \times_{f} S^{1}\right)=\left\langle\Psi_{M}|\hat{f}| \Psi_{M}\right\rangle$.
} 
The MPS diagram is shown in figure 3[a]. If $g h g^{-1}=h$, the $\mathrm{U}(1)$ factor is well-defined which is invariant under the 1-coboundary. From the TFT point of view, this invariant is nothing but the partition function $Z_{T^{2}}(g, h)$ on the torus $T^{2}$ with the background $g$ and $h$ twist

$$
\epsilon(g, h)=Z_{T^{2}}(g, h)=\left\langle\Psi_{h}|\hat{g}| \Psi_{h}\right\rangle, \quad(g h=h g) .
$$

In the spacetime path integral, it would be useful to introduce symmetry defect lines to express the background $G$ field. The matter field is transformed by $U_{g}$ when it passes through the symmetry defect line of $g$. Figure $3[\mathrm{~b}]$ shows the symmetry defect lines corresponding to the partition function $Z_{T^{2}}(g, h)$ twisted by $g$ and $h$. The discrete torsion phase $\epsilon(g, h)$ arises from the intersection of two symmetry defect lines of $g$ and $h$ with $[g, h]=0$.

Discrete torsion phase (2): partial symmetry action and swapping. There is an alternative way to detect the discrete torsion phase invariant. It is given by the combination of the partial symmetry action and the swapping operator, described as follows. Let $|\Psi\rangle$ be the ground state on $S^{1}$ with no flux. (In the TFT path integral, this state is obtained/defined by the path-integral over the disc.) We introduce three adjacent intervals $I_{1} \cup I_{2} \cup I_{3}$ with $I_{1}$ and $I_{3}$ having the same number of sites. The discrete torsion phase $\epsilon(g, h)$ is then extracted as the complex $\mathrm{U}(1)$ phase of the quantity [22]

$$
Z=\left\langle\Psi\left|\prod_{j \in I_{1} \cup I_{2}}\left(U_{h}\right)_{j} \cdot \operatorname{Swap}\left(I_{1}, I_{3}\right) \cdot \prod_{j \in I_{1} \cup I_{2}}\left(U_{g}\right)_{j}\right| \Psi\right\rangle, \quad g h=h g,
$$

in the limit $\left|I_{1}\right|,\left|I_{2}\right|,\left|I_{3}\right| \gg \xi$, where $\xi$ is the correlation length of the bulk. Here, $\operatorname{Swap}\left(I_{1}, I_{3}\right)$ is the operator swapping the two intervals $I_{1}$ and $I_{3}$, which is defined by

$$
\operatorname{Swap}\left(I_{1}, I_{3}\right)\left|m_{j}\right\rangle= \begin{cases}\left|m_{j+\left|I_{1}\right|+\left|I_{2}\right|}\right\rangle & \left(j \in I_{1}\right), \\ \left|m_{j-\left|I_{1}\right|-\left|I_{2}\right|}\right\rangle & \left(j \in I_{3}\right), \\ \left|m_{j}\right\rangle & \text { (otherwise). }\end{cases}
$$

For the MPS representation of the ground state $|\Psi\rangle$, the MPS diagram of $Z$ is written as figure 4. For the fixed point MPS (2.18), it is easy to show that $Z=|Z| \epsilon(g, h)$. The path-integral picture also verifies that $Z$ gives the discrete torsion phase. See figure 5 . Topologically, the swapping operator $\operatorname{Swap}\left(I_{1}, I_{3}\right)$ with the intermediate region $I_{2}$ is equivalent to adding a genus. The background $G$ field obtained by the partial symmetry actions $U_{g}$ and $U_{h}$ on the adjacent intervals $I_{1} \cup I_{2}$ has an intersection between two symmetry defect lines of $g$ and $h$, which leads to the discrete torsion phase $\epsilon(g, h)$.

Crosscap from inversion symmetry: "partial inversion". The crosscap topological invariant can be defined when the symmetry group $G$ includes spatial inversion or timereversal. The procedures to extract the invariant from ground state wave functions are different for spatial inversion and time-reversal. Let us first discuss the crosscap topological invariant when $G$ includes spatial inversion. 


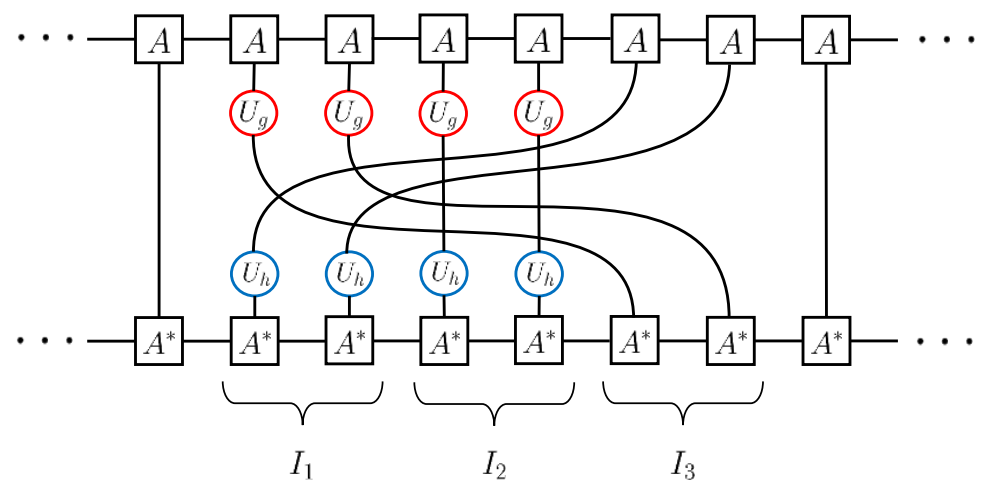

Figure 4. MPS expression of the partial symmetry action with swapping defined in (2.39).
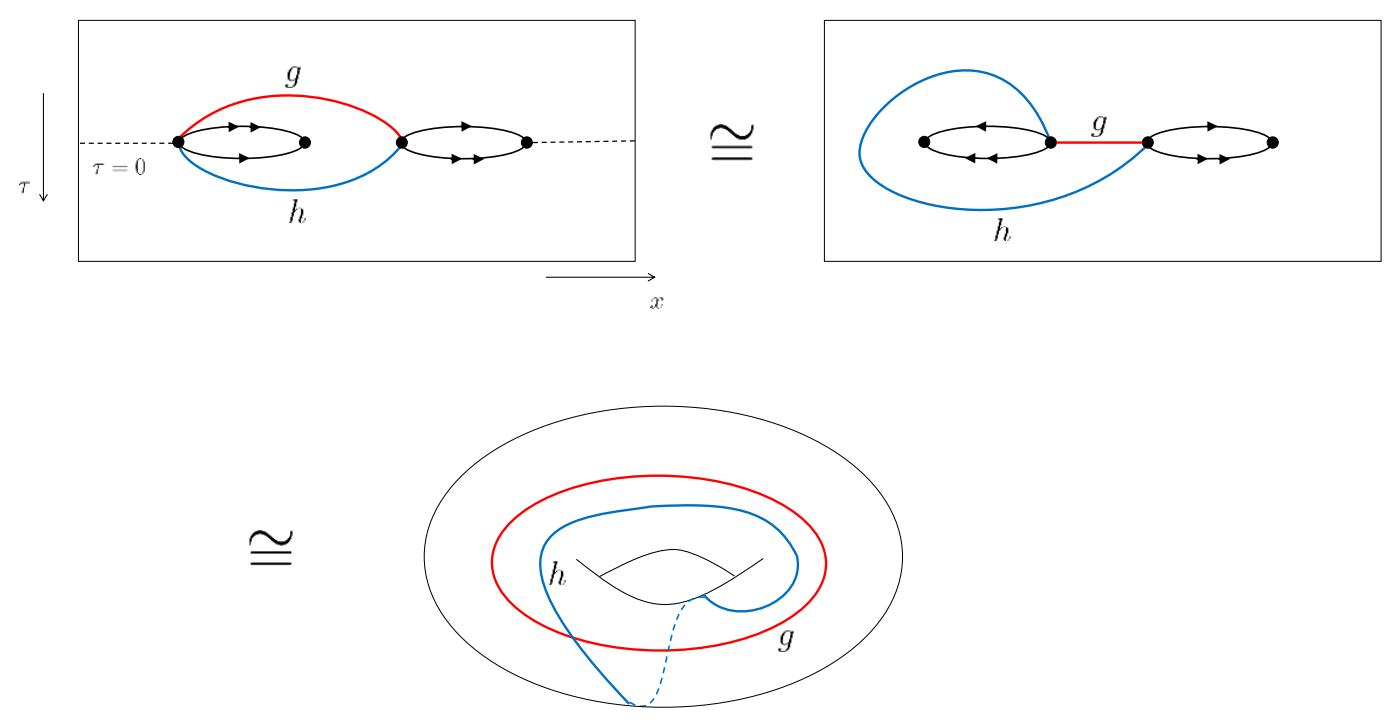

Figure 5. The geometry of path integral for the partial symmetry action with swapping operator defined in (2.39). The red and blue lines express the symmetry defect lines. The intervals with arrows are identified with ones having the same number of arrows, which results in the 2-torus.

[a]

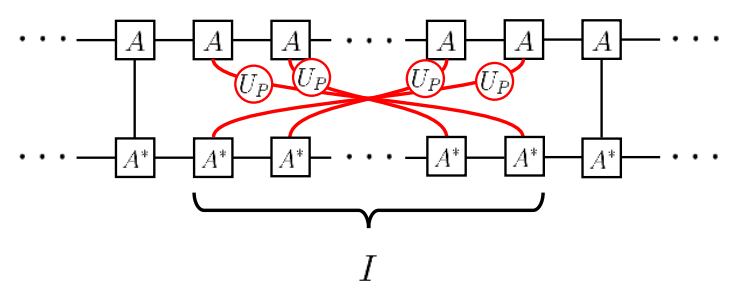

[b]

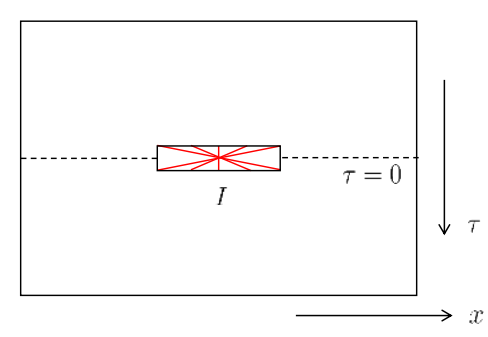

Figure 6. [a] MPS representation of crosscap. [b] The geometry of path integral for the partial inversion. 
Let $|\Psi\rangle$ be a MPS on the closed chain $L$. To create the real projective plane $\mathbb{R} P^{2}$, we take the partial inversion on the interval $I=\{1, \ldots, N\}$, as shown in figure 6 [a], as

$$
\hat{P}_{I}|\Psi\rangle=\sum_{\left\{m_{i}\right\}} \operatorname{Tr}\left(A_{m_{1}} \cdots A_{m_{L}}\right)\left|\tilde{m}_{1} \cdots \tilde{m}_{N} m_{N+1} \cdots m_{L}\right\rangle
$$

where $\widetilde{|m\rangle}$ is the partially inverted physical degrees of freedom,

$$
{\widetilde{|m\rangle_{j}}}=|n\rangle_{N-j}\left[U_{P}\right]_{n m}
$$

We assume the length of the interval $I$ is sufficiently larger than the correlation length.

The fact that this operation creates $\mathbb{R} P^{2}$ as the spacetime manifold can be easily understood from figure 6-[b]; in the path integral representation, the partial inversion is equivalent to inserting a one crosscap on the time slice at $\tau=0$.

One can show that for $P^{2}=1$ the amplitude $\hat{P}_{I}|\Psi\rangle$ gives the crosscap invariant [5]

$$
\theta(P)=Z_{\mathbb{R} P^{2}}(P)=\frac{\left\langle\Psi\left|\hat{P}_{I}\right| \Psi\right\rangle}{\left|\left\langle\Psi\left|\hat{P}_{I}\right| \Psi\right\rangle\right|}=b(P, P), \quad P^{2}=1,
$$

in the limit $L \rightarrow \infty$ and $N \rightarrow \infty$.

This formula can easily be proven for fixed point MPSs introduced in the previous section. Here we give a proof by using the cut and glue construction. [23] To illustrate the proof, we use the Haldane chain protected by the inversion symmetry with $H^{2}\left(\mathbb{Z}_{2} ; \mathrm{U}(1)_{\phi}\right)=$ $\mathbb{Z}_{2}$. First, we cut the chain $L$ by the interval $I$. There are four effective low energy degrees of freedom localized at the boundary of $I$,

$$
\left|e_{0}^{R}\right\rangle \otimes\left|e_{1}^{L}\right\rangle \otimes\left|e_{N}^{R}\right\rangle \otimes\left|e_{N+1}^{L}\right\rangle, \quad e_{j}^{L}, e_{j}^{R}=\uparrow, \downarrow .
$$

Next, we glue these degrees of freedom to get the original ground state by forming the singlet bond as sites $0-1$ and $N-(N+1)$ as

$$
|\Psi\rangle=\frac{1}{\sqrt{2}}\left(\left|\uparrow_{0}^{R}\right\rangle \otimes\left|\downarrow_{1}^{L}\right\rangle-\left|\downarrow_{0}^{R}\right\rangle \otimes\left|\uparrow_{1}^{L}\right\rangle\right) \otimes \frac{1}{\sqrt{2}}\left(\left|\uparrow_{N}^{R}\right\rangle \otimes\left|\downarrow_{N+1}^{L}\right\rangle-\left|\downarrow_{N}^{R}\right\rangle \otimes\left|\uparrow_{N+1}^{L}\right\rangle\right) .
$$

The reduced density matrix $\rho_{I}$ is

$$
\rho_{I}=\operatorname{tr}_{0, N+1}(|\Psi\rangle\langle\Psi|)=\frac{1}{4}\left[\left(\left|\uparrow_{1}^{L}\right\rangle\left\langle\uparrow_{1}^{L}|+| \downarrow_{1}^{L}\right\rangle\left\langle\downarrow_{1}^{L}\right|\right) \otimes\left(\left|\uparrow_{N}^{R}\right\rangle\left\langle\uparrow_{N}^{R}|+| \downarrow_{N}^{R}\right\rangle\left\langle\downarrow_{N}^{R}\right|\right)\right]=\frac{1}{4} \operatorname{Id}_{1}^{L} \otimes \operatorname{Id}_{N}^{R} .
$$

The partial inversion $P_{I}$ acts as

$$
\hat{P}_{I}\left|\uparrow_{1}^{L}\right\rangle=\left|\downarrow_{N}^{R}\right\rangle, \quad \hat{P}_{I}\left|\downarrow_{1}^{L}\right\rangle=-\left|\uparrow_{N}^{R}\right\rangle, \quad \hat{P}_{I}\left|\uparrow_{N}^{R}\right\rangle=\left|\downarrow_{1}^{L}\right\rangle, \quad \hat{P}_{I}\left|\downarrow_{N}^{R}\right\rangle=-\left|\uparrow_{1}^{L}\right\rangle,
$$

from which we read off

$$
\left\langle\Psi\left|\hat{P}_{I}\right| \Psi\right\rangle=\operatorname{tr}_{I}\left(\hat{P}_{I} \rho_{I}\right)=-\frac{1}{2} .
$$

We thus obtained the topological invariant $b(P, P)=-1$, which, as expected, is non-trivial (differs from $b(P, P)=1$ ), and is the $\mathbb{Z}_{2}$ invariant. 
[a]

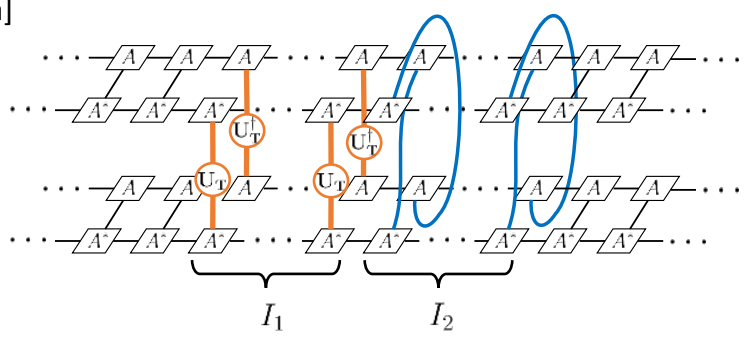

[b]

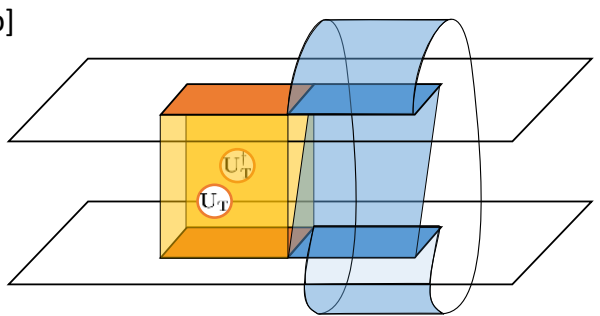

Figure 7. [a] MPS representation of the crosscap. [b] The path integral representation of partial transposition.

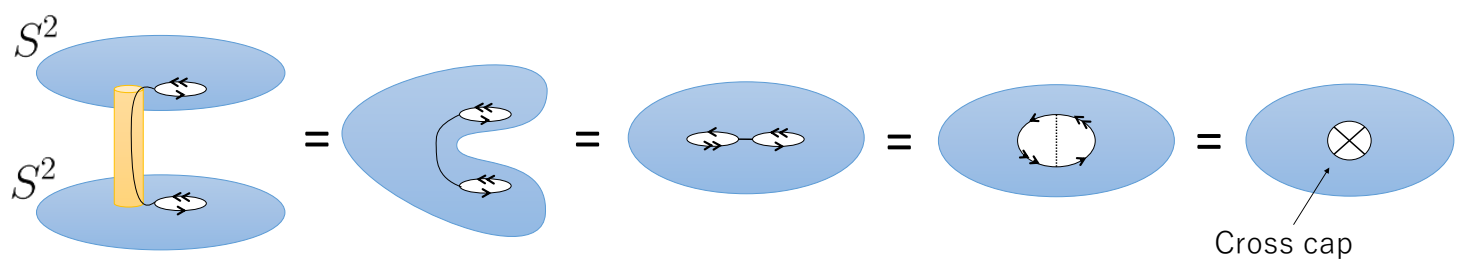

Figure 8. Equivalence between adjacent partial transpose and inserting a cross cap. The black line is identified.

Crosscap from time-reversal symmetry: "adjacent partial transposition". Next, we describe the extraction of the crosscap invariant when $G$ includes time-reversal. To this end, we will consider the so-called partial transposition. The partial transposition has been used, for example, to define the entanglement negativity. [24] Pollmann and Turner [5] showed the MPS network (figure 7 [a]) of the partial transposition on adjacent intervals $I=$ $I_{1} \cup I_{2}$ is nothing but the $\mathbb{Z}_{2}$ topological invariant $b(T, T)$ associated with the time-reversal symmetry $T \notin G_{0}$. (Here, to be concrete, let $I_{1}=\{1, \ldots, N\}$ and $I_{2}=\{N+1, \ldots, M\}$ be two adjacent intervals in the chain of length $L$.) In the following, we will review the topological invariant of Pollmann and Turner. We will also note, with an eye toward the TFT descriptions of SPT phases and their topological invariants, that the Pollmann-Turner can be interpreted as a space-time path integral on the real projective plane.

Below, we will express the Pollmann-Turner invariant in terms of a density matrix. We introduce the reduced density matrix for the interval $I$ by taking the partial trace of the degrees of freedom on living on the compliment of $I, L \backslash I$, as

$$
\rho_{I}=\operatorname{tr}_{L \backslash I}(|\Psi\rangle\langle\Psi|)
$$

The reduced density matrix $\rho_{I}$ is expanded in the basis of $I_{1}$ and $I_{2}$ as

$$
\rho_{I}=\sum_{i j k l}\left|e_{i}^{1}, e_{j}^{2}\right\rangle\left\langle e_{i}^{1}, e_{j}^{2}\left|\rho_{I}\right| e_{k}^{1}, e_{l}^{2}\right\rangle\left\langle e_{k}^{1}, e_{l}^{2}\right|
$$

where $\left|e_{i}^{1}\right\rangle$ and $\left|e_{j}^{2}\right\rangle$ are the basis on the intervals $I_{1}$ and $I_{2}$, respectively. We introduce the 
partial transposition $\rho_{I}^{T_{1}}$ for the interval $I_{1}$ which is defined by [24]

$$
\rho_{I}^{T_{1}}:=\sum_{i j k l}\left|e_{i}^{1}, e_{j}^{2}\right\rangle\left\langle e_{k}^{1}, e_{j}^{2}\left|\rho_{I}\right| e_{i}^{1}, e_{l}^{2}\right\rangle\left\langle e_{k}^{1}, e_{l}^{2}\right| .
$$

In addition to the partial transposition, we also consider the "unitary part" of time-reversal, and consider partial time-reversal transformation $\hat{T}_{I_{1}}^{\prime}=\prod_{j \in I_{1}}\left(U_{T}\right)_{j}$ action only on $I_{1}$. Note that $\hat{T}_{I_{1}}^{\prime}$ is unitary, i.e., it consists of the only unitary part of the time-reversal transformation $\hat{T}=\left(\prod_{j}\left(U_{T}\right)_{j}\right) K$, where $K$ is complex conjugation. Putting everything together, we consider

$$
\operatorname{tr}_{I}\left(\rho_{I} \hat{T}_{I_{1}}^{\prime} \rho_{I}^{T_{1}}\left[\hat{T}_{I_{1}}^{\prime}\right]^{\dagger}\right), \quad \hat{T}_{I_{1}}^{\prime}=\prod_{j \in I_{1}}\left(U_{T}\right)_{j} .
$$

Finally, the Pollmann-Turner topological invariant, i.e., the crosscap topological invariant is given by the phase of $(2.52)$,

$$
\theta(T)=\frac{\operatorname{tr}_{I}\left(\rho_{I} \hat{T}_{I_{1}}^{\prime} \rho_{I}^{T_{1}}\left[\hat{T}_{I_{1}}^{\prime}\right]^{\dagger}\right)}{\left|\operatorname{tr}_{I}\left(\rho_{I} \hat{T}_{I_{1}}^{\prime} \rho_{I}^{T_{1}}\left[\hat{T}_{I_{1}}^{\prime}\right]^{\dagger}\right)\right|} \rightarrow b(T, T), \quad(N, M \rightarrow \infty) .
$$

In the limit $N, M \gg \xi$, where $\xi$ the correlation length, the phase $\theta(T)$ is a quantized topological invariant.

The path integral representation of the quantity (2.52) is shown in figure $7[\mathrm{~b}]$, which is topologically equivalent to a sphere with one crosscap as shown in figure 8 .

That (2.53) is indeed a quantized and topological invariant can be proven within the MPS framework. Here, we demonstrate this by again using the cut and glue construction [23], and by taking the Haldane chain with time-reversal symmetry as an example. Within the cut and glue construction, there are six active degrees of freedom at low energies in the reduced density matrix,

$$
\left|e_{0}^{R}\right\rangle \otimes\left|e_{1}^{L}\right\rangle \otimes\left|e_{N}^{R}\right\rangle \otimes\left|e_{N+1}^{L}\right\rangle \otimes\left|e_{M}^{R}\right\rangle \otimes\left|e_{M+1}^{L}\right\rangle, \quad e_{j}^{L}, e_{j}^{R}=\uparrow, \downarrow .
$$

The ground state is a singlet formed from $\left(\left|e_{0}^{R}\right\rangle,\left|e_{1}^{L}\right\rangle\right),\left(\left|e_{N}^{R}\right\rangle,\left|e_{N+1}^{L}\right\rangle\right)$ and $\left(\left|e_{M}^{R}\right\rangle,\left|e_{M+1}^{L}\right\rangle\right)$ as

$$
\begin{aligned}
|\Psi\rangle= & \frac{1}{\sqrt{2}}\left(\left|\uparrow_{0}^{R}\right\rangle \otimes\left|\downarrow_{1}^{L}\right\rangle-\left|\downarrow_{0}^{R}\right\rangle \otimes\left|\uparrow_{1}^{L}\right\rangle\right) \otimes \frac{1}{\sqrt{2}}\left(\left|\uparrow_{N}^{R}\right\rangle \otimes\left|\downarrow_{N+1}^{L}\right\rangle-\left|\downarrow_{N}^{R}\right\rangle \otimes\left|\uparrow_{N+1}^{L}\right\rangle\right) \\
& \otimes \frac{1}{\sqrt{2}}\left(\left|\uparrow_{M}^{R}\right\rangle \otimes\left|\downarrow_{M+1}^{L}\right\rangle-\left|\downarrow_{M}^{R}\right\rangle \otimes\left|\uparrow_{M+1}^{L}\right\rangle\right) .
\end{aligned}
$$

The reduced density matrix $\rho_{I}$ reads

$$
\begin{aligned}
\rho_{I} & =\operatorname{tr}_{0, M+1}(|\Psi\rangle\langle\Psi|) \\
= & \frac{1}{2} \operatorname{Id}_{1}^{L} \otimes \frac{1}{2}\left[\left|\uparrow_{N}^{R}\right\rangle\left\langle\uparrow_{N}^{R}|\otimes| \downarrow_{N+1}^{L}\right\rangle\left\langle\downarrow_{N+1}^{L}|+| \downarrow_{N}^{R}\right\rangle\left\langle\downarrow_{N}^{R}|\otimes| \uparrow_{N+1}^{L}\right\rangle\left\langle\uparrow_{N+1}^{L}\right|\right. \\
& \left.\quad-\left|\uparrow_{N}^{R}\right\rangle\left\langle\downarrow_{N}^{R}|\otimes| \downarrow_{N+1}^{L}\right\rangle\left\langle\uparrow_{N+1}^{L}|-| \downarrow_{N}^{R}\right\rangle\left\langle\uparrow_{N}^{R}|\otimes| \uparrow_{N+1}^{L}\right\rangle\left\langle\downarrow_{N+1}^{L}\right|\right] \otimes \frac{1}{2} \operatorname{Id}_{M}^{R} .
\end{aligned}
$$

By taking the partial transposition on $I_{1}=\{1, N\}$ and noting that the unitary part of the time-reversal transformation is given by

$$
U_{T}|\uparrow\rangle=|\downarrow\rangle, \quad U_{T}|\downarrow\rangle=-|\uparrow\rangle,
$$


we have

$$
\begin{aligned}
\hat{T}_{I_{1}}^{\prime} \rho_{I}^{T_{1}}\left[\hat{T}_{I_{1}}^{\prime}\right]^{\dagger}= & \frac{1}{2} \operatorname{Id}_{1}^{L} \otimes \frac{1}{2}\left[\left|\downarrow_{N}^{R}\right\rangle\left\langle\downarrow_{N}^{R}|\otimes| \downarrow_{N+1}^{L}\right\rangle\left\langle\downarrow_{N+1}^{L}|+| \uparrow_{N}^{R}\right\rangle\left\langle\uparrow_{N}^{R}|\otimes| \uparrow_{N+1}^{L}\right\rangle\left\langle\uparrow_{N+1}^{L}\right|\right. \\
& \left.+\left|\uparrow_{N}^{R}\right\rangle\left\langle\downarrow_{N}^{R}|\otimes| \downarrow_{N+1}^{L}\right\rangle\left\langle\uparrow_{N+1}^{L}|+| \downarrow_{N}^{R}\right\rangle\left\langle\uparrow_{N}^{R}|\otimes| \uparrow_{N+1}^{L}\right\rangle\left\langle\downarrow_{N+1}^{L}\right|\right] \otimes \frac{1}{2} \operatorname{Id}_{M}^{R},
\end{aligned}
$$

which leads to

$$
\operatorname{tr}_{I}\left(\rho_{I} \hat{T}_{I_{1}}^{\prime} \rho_{I}^{T_{1}}\left[\hat{T}_{I_{1}}^{\prime}\right]^{\dagger}\right)=-\frac{1}{8}
$$

The minus sign $(-1)$ is the proper $\mathbb{Z}_{2}$ invariant for the Haldane chain with time-reversal symmetry.

Klein bottle partition function from inversion symmetry. Similar to the crosscap topological invariant, the Klein bottle topological invariant can be defined both for spatial inversion and time-reversal. Let us start with the case of spatial inversion.

We act with an inversion transformation $P \notin G_{0}$ on the twisted MPS $\left|\Psi_{g}\right\rangle$,

$$
\hat{P}\left|\Psi_{g}\right\rangle=\sum_{\left\{m_{i}\right\}} \operatorname{Tr}\left(A_{m_{1}} \cdots A_{m_{L}} V_{P} V_{g}^{T} V_{P}^{-1}\right)\left|m_{1} \cdots m_{L}\right\rangle=\frac{b\left(P, g^{-1}\right) b\left(g, g^{-1}\right)}{b\left(P g^{-1} P^{-1}, P\right)}\left|\Psi_{P g^{-1} P^{-1}}\right\rangle .
$$

If $P g^{-1} P^{-1}=g$, the $\mathrm{U}(1)$ prefactor is well-defined which is invariant under the 1coboundary. This invariant is nothing but the partition function $Z_{K B}(P ; g)$ over the Klein bottle $(K B)$ with the background $P$ and $g$ twists

$$
\kappa(P ; g)=Z_{K B}(P ; g)=\left\langle\Psi_{g}|\hat{P}| \Psi_{g}\right\rangle, \quad\left(P \notin G_{0}, g \in G_{0}, P g^{-1}=g P\right) .
$$

An example is a $\mathbb{Z}_{2}(=\{1, \sigma\})$ paramagnet with inversion symmetry $\mathbb{Z}_{2}^{P}(=\{1, P\})$ where $\mathbb{Z}_{2}$ charge is preserved under the inversion. The topological classification is given by $H^{2}\left(\mathbb{Z}_{2} \times \mathbb{Z}_{2}^{P}, \mathrm{U}(1)_{\phi}\right)=\mathbb{Z}_{2} \times \mathbb{Z}_{2}$. The two topological invariants can be seen in $V_{P} V_{P}^{*}=\theta(P)$ and $V_{P} V_{\sigma}^{T}=\kappa(P ; \sigma) V_{\sigma} V_{P}$.

Klein bottle partition function from time-reversal symmetry: "disjoint partial transposition with intermediate twist". Next, as for time-reversal symmetry, the Klein bottle partition function obtained from from time-reversal can be represented in terms of MPSs in a way similar to the crosscap partition function. First, we divide the closed chain $L$ into three adjacent intervals $I_{1} \cup I_{2} \cup I_{3}, I_{1}=\left\{1, \ldots, N_{1}\right\}, I_{2}=\left\{N_{1}+1, \ldots, N_{2}\right\}$, $I_{3}=\left\{N_{2}+1, \ldots, N_{3}\right\}$. In addition, we introduce one replica. We trace out the region except for $I_{1} \cup I_{3}$ with symmetry twist in the interval $I_{2}$ as

$$
\rho_{I_{1} \cup I_{3}}(g):=\operatorname{tr}_{L \backslash\left(I_{1} \cup I_{3}\right)}\left(\hat{g}_{I_{2}}|\Psi\rangle\langle\Psi|\right), \quad \hat{g}_{I_{2}}=\prod_{j \in I_{2}}\left(U_{g}\right)_{j} .
$$

Then, we consider the following quantity

$$
\operatorname{tr}_{I_{1} \cup I_{3}}\left(\rho_{I_{1} \cup I_{3}}(g) \hat{T}_{I_{1}}^{\prime} \rho_{I_{1} \cup I_{3}}\left(g^{-1}\right)\left[\hat{T}_{I_{1}}^{\prime}\right]^{\dagger}\right), \quad\left(T g^{-1} T^{-1}=g\right), \quad T_{I_{1}}^{\prime}=\prod_{j \in I_{1}}\left(U_{T}\right)_{j} .
$$




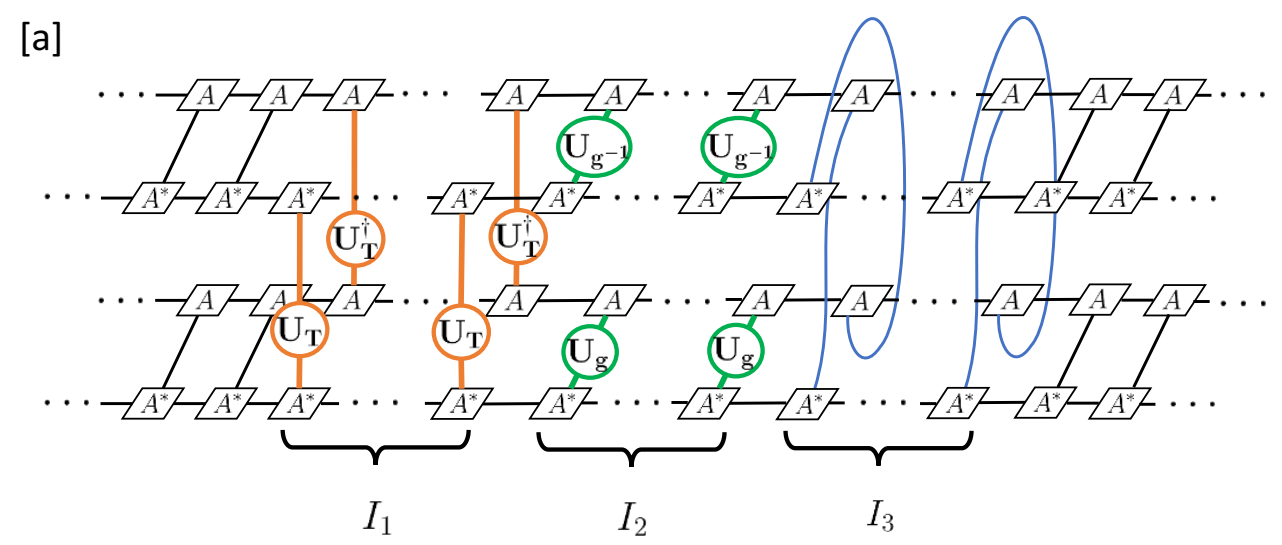

[b]

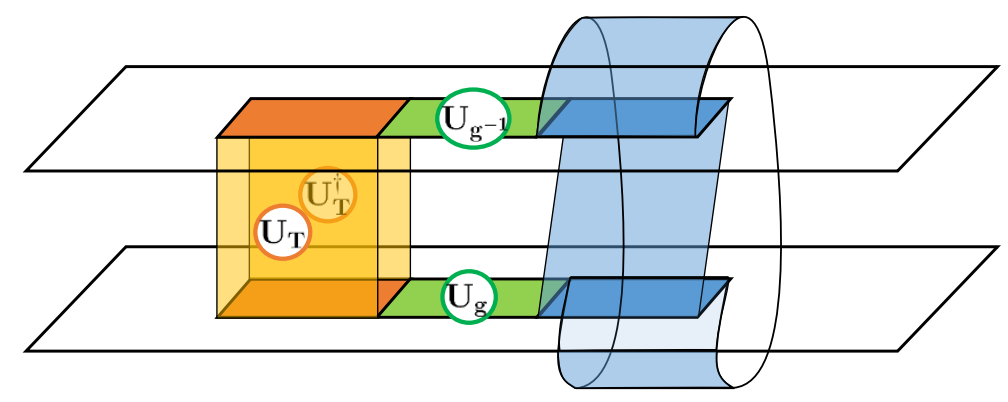

Figure 9. [a] MPS representation of the Klein bottle partition function with twist. [b] The path integral representation.

The corresponding MPS network and path integral are shown in figure 9 [a] and [b], respectively. One can show this quantity approaches the Klein bottle partition function

$$
\frac{\operatorname{tr}_{I_{1} \cup I_{3}}\left(\rho_{I_{1} \cup I_{3}}(g) \hat{T}_{I_{1}}^{\prime} \rho_{I_{1} \cup I_{3}}\left(g^{-1}\right)\left[\hat{T}_{I_{1}}^{\prime}\right]^{\dagger}\right)}{\left|\operatorname{tr}_{I_{1} \cup I_{3}}\left(\rho_{I_{1} \cup I_{3}}(g) \hat{T}_{I_{1}}^{\prime} \rho_{I_{1} \cup I_{3}}\left(g^{-1}\right)\left[\hat{T}_{I_{1}}^{\prime}\right]^{\dagger}\right)\right|} \rightarrow Z_{K B}(T ; g)=\kappa(T ; g)
$$

in the limit $N_{1}, N_{2}-N_{1}, N_{3}-N_{2}, L-N_{3} \gg \xi$, where $\xi$ is the correlation length. It is easy to show the above formula for fixed point MPSs by using the symmetry properties of $A$ matrix.

\section{G-equivariant topological field theories and MPSs}

Having discussed the MPS description of $(1+1)$ d bosonic SPT phases, we now move on $(1+1) \mathrm{d} G$-equivaliant TFTs. In the following sections, section 3.1 to section 3.4, we briefly summarize necessary ingredients of open and closed $G$-equivariant oriented $(1+1) \mathrm{d}$ TFTs following Moore-Segal [11]. There are some overlaps with ref. [12], where they also discuss closed $G$-equivariant $(1+1)$ d unoriented TFTs. Ref. [11] also discusses $(1+1)$ d open and closed TFTs with spin structure, which can describe fermionic SPT phases such as class D topological superconductors. Here we restrict ourselves to $(1+1)$ d bosonic SPT 


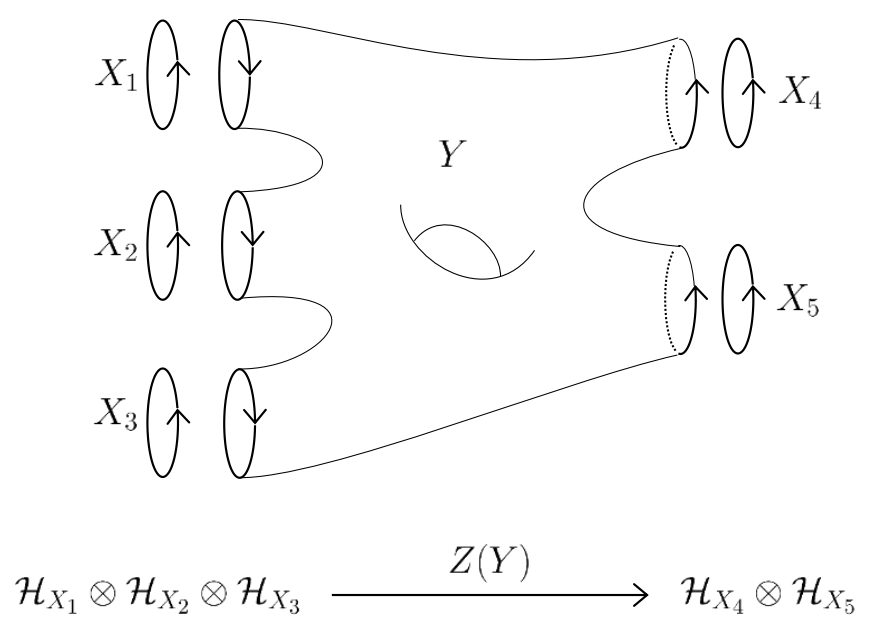

Figure 10. An example of cobordism.

phase protected by on-site unitary $G$-symmetry where $G$ is a finite group. In short, a $G$ equivariant TFT is a TFT couped with the background $G$-gauge field. (Integrating out the background $G$-gauge field, i.e. orbifolding the $G$-symmetry, leads to an orbifolded theory which is a TFT without $G$-symmetry.)

In the following, first, we introduce some general properties of TFTs. Next, we summarize $(1+1) \mathrm{d} G$-equivariant closed TFTs with an eye toward $(1+1) \mathrm{d}$ SPT phases. Our notations closely follow Moore-Segal [11]. Next, we will summarize $(1+1) d$ d-equivariant open and closed TFTs.

\subsection{Some basics of TFTs}

In the axiomatic definition, a TFT in $(d+1)$ dimensions is a functor $Z$ from a cobordism category Bord $_{\langle d, d+1>}$ to the category of finite dimensional complex vector spaces Vect equipped with tensor product. [25, 26] In Bord $_{<d, d+1>}$, objects are $d$-dimensional manifolds $X_{1}, X_{2}, \ldots$, and morphism is a cobordism $Y: X_{1} \rightarrow X_{2}$ which is a manifold of dimension $d+1$ and has $X_{1}$ and $X_{2}$ as its boundary components, $\partial Y=\left(-X_{1}\right) \sqcup X_{2}$, where $(-X)$ is $X$ with opposite orientation. In general, we can associate a structure (e.g. spin structure for spin TFTs, background gauge field for equivariant TFTs, etc.) with manifolds. For each $d$-dimensional manifold $X$, we associate a Hilbert space $\mathcal{H}_{X}$ by a functor $Z$. A direct sum of manifolds $X_{1} \sqcup X_{2} \sqcup \cdots$ is mapped into a tensor product $\mathcal{H}_{X_{1}} \otimes \mathcal{H}_{X_{2}} \otimes \cdots$. A cobordism $Y$ between $X$ and $X^{\prime}$ leads to a linear map $Z(Y): \mathcal{H}_{X} \rightarrow \mathcal{H}_{X^{\prime}}$. See figure 10, for an example.

In any TFT, the cylinder cobordism $X \times I$ leads to the identity map $Z(X \times I)=$ id

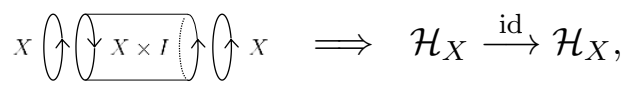

which is equivalent to the fact that the Hamiltonian of TFTs is zero. 
In addition, we have a bilinear form $Q$ and a coform $\Delta$ :
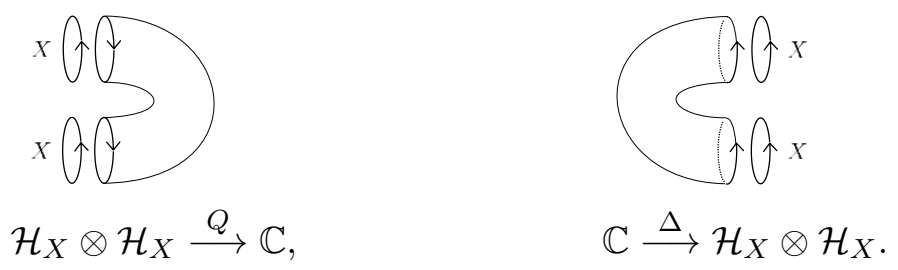

Let $\left\{\phi_{i}\right\}$ be a basis of $\mathcal{H}_{X}$ and write $Q\left(\phi_{i}, \phi_{j}\right)=Q_{i j}, \Delta(1)=\sum_{i j} \Delta_{i j} \phi_{i} \otimes \phi_{j}$. The equivalence between the "S-tube" and the cylinder,

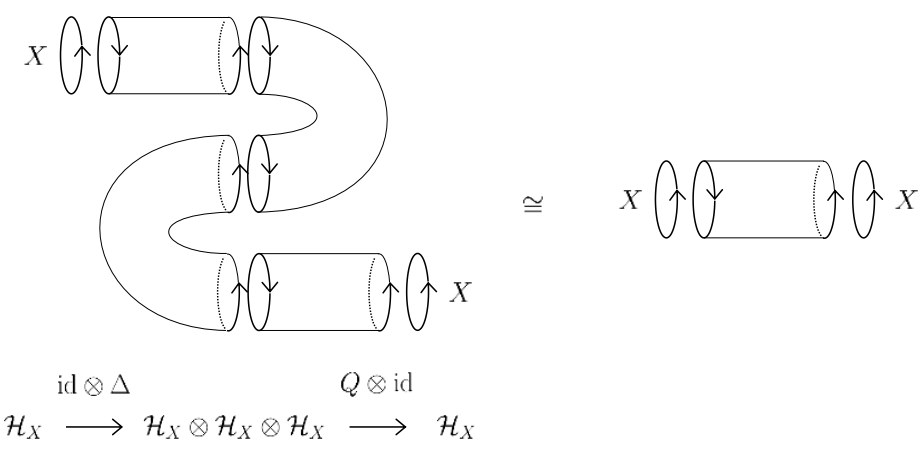

implies

$$
\phi \mapsto \phi \otimes \sum_{j k} \Delta_{j k} \phi_{j} \otimes \phi_{k} \mapsto \sum_{i} Q\left(\phi, \phi_{j}\right) \Delta_{j k} \phi_{k}=\phi, \quad \phi \in \mathcal{H}_{X}
$$

By setting $\phi=\phi_{k}$, we have $\sum_{j} Q_{i j} \Delta_{j k}=\delta_{i k}$, which means $Q$ is nondegenerate. Choosing the basis so that $Q_{i j}=\delta_{i j}$, in this basis the coform $\Delta$ is simply $\Delta=\sum_{i} \phi_{i} \otimes \phi_{i}$.

\section{$3.2 \quad G$-equivariant oriented closed TFTs}

A $G_{0}$-equivariant oriented $(1+1) \mathrm{d}$ TFT is a functor $Z$ from a cobordism category with a background $G_{0}$ gauge field to the category of complex vector spaces. (To distinguish on-site unitary symmetries from orientation-reversing symmetries, here we use a notation $G_{0}$ to denote on-site unitary symmetries.) For $(1+1)$ d TFTs, the minimum object is an oriented circle $\left(S^{1}, p t, g\right)$ with background $g \in G_{0}$ flux together with a trivialization at a base point $p t \in S^{1}$, which is specified by a twisted boundary condition by an element $g \in G_{0}$ at $p t$. We denote the Hilbert space associated with $\left(S^{1}, p t, g \in G_{0}\right)$ by $\mathcal{C}_{g}$ :

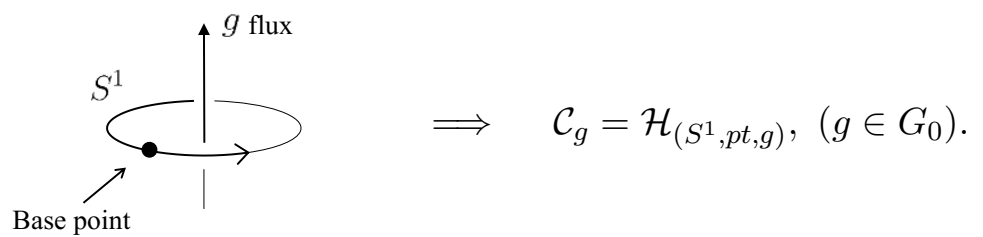

For an unpointed circle $S^{1}$, a background flux inserted in the circle $S^{1}$ is characterized by a conjugacy class $[g]=\left\{h g h^{-1} \mid h \in G\right\}$ rather than an element $g \in G_{0}$. For point circles 
[a]

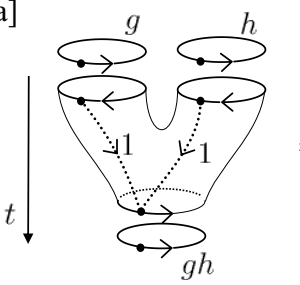

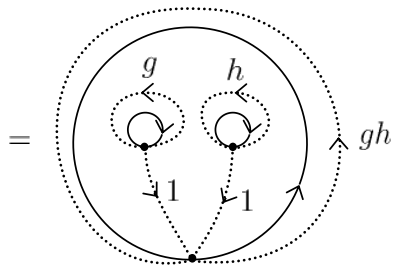

[b]

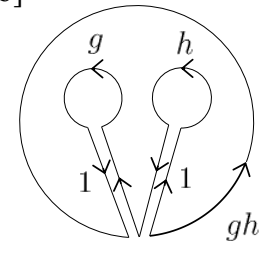

Figure 11. [a] A fusion process $\mathcal{C}_{g} \otimes \mathcal{C}_{h} \rightarrow \mathcal{C}_{g h}$. Dashed lines with group elements represent holonomies. The figure [b] shows a holonomy around the boundary of simply connected space that is obtained by cutting the surface in [a] at the lines connecting base points.

$\left(S^{1}, p t\right)$ with trivialization of background $G_{0}$ gauge field at $p t$, Hilbert spaces are labeled by elements $g \in G_{0}$. We have thus a $G_{0}$-graded Hilbert space

$$
\mathcal{C}=\bigoplus_{g \in G_{0}} \mathcal{C}_{g}
$$

In graphical representations of morphisms, we specify the background gauge field by holonomies connecting base points on initial and mapped circles. For example, the fusion process of two circles with $g$ and $h$ fluxes is represented in figure 11 [a]. The $G_{0}$ flux of mapped pointed circle $\left(S^{1}, p t\right)$ is determined by holonomies along base points as shown in figure $11[\mathrm{~b}]$. Recall that a holonomy around a boundary of simply connected spaces is trivial. In short, we simply write the bordsim of the fusion process as

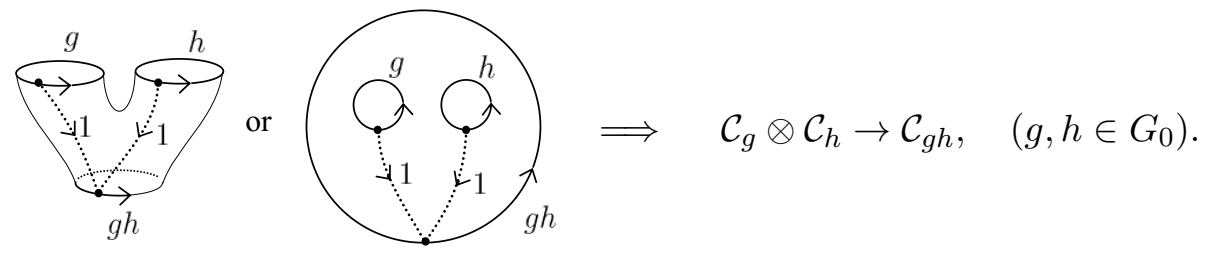

In table 1 , we show building blocks of $G_{0}$-equivariant oriented $(1+1)$ d TFTs. All other cobordisms and partition functions can be constructed by processes in table 1. For example, the "branching" process is given by

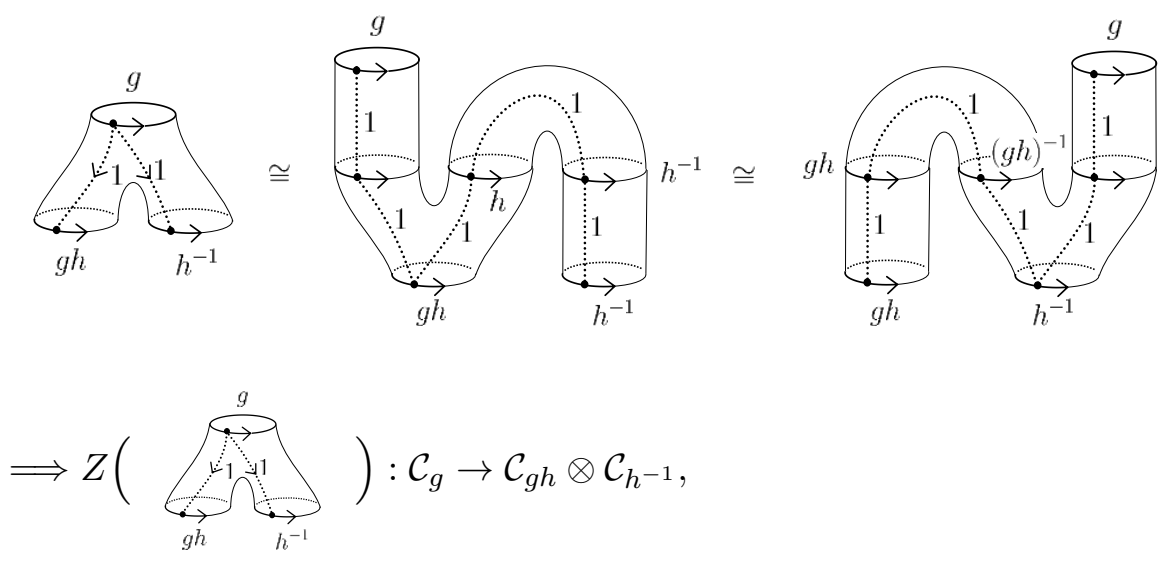




\begin{tabular}{|c|c|c|c|c|}
\hline & Manifolds & Hilbert spaces & Simple and fixed point MPS & Comment \\
\hline (a) & $\stackrel{g}{\longrightarrow}$ & $\mathcal{C}_{g}\left(g \in G_{0}\right)$ & $\begin{array}{c}\text { Hilbert space generated by } \\
\left|\Psi_{g}\right\rangle=\operatorname{tr}\left(A_{m} V_{g}\right)|m\rangle, g \in G_{0}\end{array}$ & $\begin{array}{l}\text { Hilbert space over a space circle } \\
\text { with } g \text {-flux }\end{array}$ \\
\hline (b) & & $\begin{array}{l}\theta_{\mathcal{C}}: \mathcal{C}_{1} \rightarrow \mathbb{C} \\
\phi \mapsto \theta_{\mathcal{C}}(\phi)\end{array}$ & $\theta_{\mathcal{C}}\left(\operatorname{tr}\left(A_{m}\right)|m\rangle\right)=1$ & $\begin{array}{c}Q\left(\phi_{1}, \phi_{2}\right):=\theta_{\mathcal{C}}\left(\phi_{1} \phi_{2}\right),\left(\phi_{1} \in\right. \\
\left.\mathcal{C}_{g}, \phi_{2} \in \mathcal{C}_{g^{-1}}\right) \text { is a bilinear } \\
\text { nondegenerate form }\end{array}$ \\
\hline (c) & & $\begin{array}{l}\mathbb{C} \rightarrow \mathcal{C}_{1} \\
1 \mapsto 1_{\mathcal{C}}\end{array}$ & $1_{\mathcal{C}}=\operatorname{tr}\left(A_{m}\right)|m\rangle$ & $\begin{array}{l}\text { State on the boundary of disc } \\
\qquad 1_{\mathcal{C}} \phi=\phi 1_{\mathcal{C}}=\phi\end{array}$ \\
\hline (d) & $h$ & $\begin{aligned} \alpha_{g \in G_{0}}: & \mathcal{C}_{h} \rightarrow \mathcal{C}_{g h g^{-1}} \\
& \phi \mapsto \alpha_{g}(\phi)\end{aligned}$ & $\begin{array}{l}\operatorname{tr}\left(A_{m} V_{h}\right)|m\rangle \\
\mapsto \operatorname{tr}\left(A_{m} V_{h}\right) \hat{g}(|m\rangle) \\
=\operatorname{tr}\left(A_{m} V_{g} V_{h} V_{g}^{\dagger}\right)|m\rangle\end{array}$ & $\begin{array}{c}\text { On site unitary } g \in G_{0} \text { symmetry } \\
\text { action }\end{array}$ \\
\hline (e) & $g h$ & $\begin{array}{l}\mathcal{C}_{g} \otimes \mathcal{C}_{h} \rightarrow \mathcal{C}_{g h} \\
\phi_{1} \otimes \phi_{2} \mapsto \phi_{1} \phi_{2}\end{array}$ & $\begin{array}{l}\operatorname{tr}\left(A_{m_{1}} V_{g}\right)\left|m_{1}\right\rangle \\
\otimes \operatorname{tr}\left(A_{m_{2}} V_{h}\right)\left|m_{2}\right\rangle \\
\mapsto \operatorname{tr}\left(A_{m} V_{g} V_{h}\right)|m\rangle\end{array}$ & "Fusion" of two closed chains \\
\hline (f) & 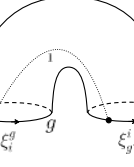 & $\begin{array}{l}\Delta_{g}: \mathbb{C} \rightarrow \mathcal{C}_{g} \otimes \mathcal{C}_{g^{-1}} \\
\Delta_{g}(1)=\sum_{i} \xi_{i}^{g} \otimes \xi_{i}^{g^{-1}}\end{array}$ & $\operatorname{tr}\left(A_{m_{1}} V_{g}\right)\left|m_{1}\right\rangle \otimes \operatorname{tr}\left(A_{m_{2}} V_{g^{-1}}\right)\left|m_{2}\right\rangle$ & $\begin{array}{c}\xi_{i}^{g} \in \mathcal{C}_{g} \text { are basis of } \mathcal{C}_{g} \text { and } \\
\xi_{i}^{g^{-1}} \in \mathcal{C}_{g^{-1}} \text { are their dual basis of } \\
\mathcal{C}_{g^{-1}} \text { that satisfy } \\
\theta_{\mathcal{C}}\left(\xi_{i}^{g} \xi_{j}^{g^{-1}}\right)=\theta_{\mathcal{C}}\left(\xi_{j}^{g^{-1}} \xi_{i}^{g}\right)=\delta_{i j} .\end{array}$ \\
\hline (g) & & $\begin{array}{l}\mathbb{C} \rightarrow \mathcal{C}_{g^{2}}, g \notin G_{0} \\
1 \mapsto \theta_{g}\end{array}$ & $b(g, g) \operatorname{tr}\left(A_{m} V_{g^{2}}\right)|m\rangle$ & $\begin{array}{l}\text { State on the boundary state of } \\
\text { Möbius strip, "cross cap state". }\end{array}$ \\
\hline (h) & $\frac{g+}{g h g^{-1}}$ & $\alpha_{g \notin G_{0}}: \mathcal{C}_{h} \rightarrow \mathcal{C}_{g h^{-1} g^{-1}}$ & $\begin{array}{l}\operatorname{tr}\left(A_{m} V_{h}\right)|m\rangle \\
\mapsto \operatorname{tr}\left(A_{m} V_{h}\right) \hat{g}(|m\rangle) \\
=\operatorname{tr}\left(A_{m}^{T} V_{g} V_{h} V_{g}^{\dagger}\right)|m\rangle\end{array}$ & $g$ reflection \\
\hline
\end{tabular}

Table 1. Building blocks of $G$-equivariant closed $(1+1) d$ TFTs. Building blocks (a)-(f) define $G$-equivariant oriented TFTs in $(1+1)$ d. G-equivariant unoriented TFTs in $(1+1)$ d are defined by including (g) and (h), in addition to (a)-(f). $G_{0} \subset G$ represents orientation preserving symmetries. The fourth column shows corresponding simple and fixed point MPS representations (see section 3.3.2).

$$
\phi \mapsto \sum_{i} \phi \xi_{i}^{h} \otimes \xi_{i}^{h^{-1}}=\sum_{i} \xi_{i}^{g h} \otimes \xi_{i}^{(g h)^{-1}} \phi, \quad\left(g, h \in G_{0}\right)
$$

Here, we made use of Items (e) and (f) in table 1 , and $\Delta_{g}(1)=\sum_{i} \xi_{i}^{g} \otimes \xi_{i}^{g^{-1}} \in \mathcal{C}_{g} \otimes \mathcal{C}_{g^{-1}}$ is the coform defined in Item (f).

The fusion process (e), which, by an axiom of TFTs, is associated to a map $\mathcal{C}_{g} \otimes \mathcal{C}_{h} \rightarrow$ $\mathcal{C}_{g h}$, makes the Hilbert space $\mathcal{C}$ into an algebra. There are several constraints on the algebra, which are obtained, e.g., by considering different factorizations of surfaces into building blocks in table 1. Due to Turaev, [8] we have the minimum defining algebraic relations $[11,12]$ : to give a $G_{0}$-equivariant oriented TFT is equivalent to give a $G_{0}$-graded 
algebra $\mathcal{C}=\bigoplus_{g \in G_{0}} \mathcal{C}_{g}$ together with a group homomorphism $\alpha: G_{0} \rightarrow \operatorname{Aut}(\mathcal{C})$ such that $\operatorname{Aut}(\mathcal{C}) \ni \alpha_{g}: \mathcal{C}_{h} \rightarrow \mathcal{C}_{g h g^{-1}}$, and

(1) There is a $G_{0}$-invariant trace $\theta_{\mathcal{C}}: \mathcal{C}_{1} \rightarrow \mathbb{C}, \theta_{\mathcal{C}} \circ \alpha_{g}=\theta_{\mathcal{C}}$, such that the induced paring $\mathcal{C}_{g} \otimes \mathcal{C}_{g^{-1}} \rightarrow \mathbb{C}$ is nondegenerate.

(2) For $\phi \in \mathcal{C}_{g}, \alpha_{g}(\phi)=\phi$.

(3) For $\phi_{1} \in \mathcal{C}_{g_{1}}, \phi_{2} \in \mathcal{C}_{g_{2}}, \alpha_{g_{2}}\left(\phi_{1}\right) \phi_{2}=\phi_{2} \phi_{1}$.

(4) (Punctured Torus) $\sum_{i} \alpha_{h}\left(\xi_{i}^{g}\right) \xi_{i}^{g^{-1}}=\sum_{i} \xi_{i}^{h} \alpha_{g}\left(\xi_{i}^{h^{-1}}\right) \in \mathcal{C}_{h g h^{-1} g^{-1}}$.

The non-degenerate property in (1) is followed by the same way as (2). Derivations of (1-4) are summarized in appendix D.1.

The state in (4) is the handle adding operator

$$
h g h^{-1}+\overbrace{i}^{h} \alpha_{h}\left(\xi_{i}^{g}\right) \xi_{i}^{g^{-1}}
$$

which enables us to compute all possible partition functions on surfaces of genus $g$ with twist. For example, the partition function on torus $T^{2}$ with twist is given by

$$
h g h^{-1}
$$

\subsubsection{General solution for semi simple cases}

If $\mathcal{C}_{1}$, the untwisted sector Hilbert space, is semisimple, $\mathcal{C}_{1} \cong \bigoplus_{x \in X} \mathbb{C} \epsilon_{x}, \epsilon_{x} \epsilon_{y}=\delta_{x, y} \epsilon_{x}$, we have general solutions for the algebraic constraints (1-4) as follows. [8, 11] Here, $X$ is a finite set equipped with $G_{0}$-action $g \cdot(h \cdot x)=(g h) \cdot x$.

For a given $G_{0}$-set $X$, the twisted sector Hilbert space $\mathcal{C}_{g}$ consists of little group at $x$ as $\mathcal{C}_{g}=\bigoplus_{x \in X, g \cdot x=x} L_{g, x}$, where $L_{g, x} \cong \mathbb{C}$ are lines. The multiplication of $\mathcal{C}=\bigoplus_{g \in G_{0}} \mathcal{C}_{g}$ is determined by a given group cocycle $b_{x}(g, h) \in Z^{2}\left(G_{0}, C(X, \mathrm{U}(1))\right)\left(\cong Z_{G_{0}}^{2}(X, \mathrm{U}(1))\right) \mathrm{as}^{2}$

$$
\ell_{g_{2}, x_{2}} \ell_{g_{1}, x_{1}}=\left\{\begin{array}{ll}
b_{x_{1}}\left(g_{2}, g_{1}\right) \ell_{g_{2} g_{1}, x_{1}} & \left(x_{2}=g_{1} \cdot x_{1}\right) \\
0 & \text { (otherwise) }
\end{array} \quad\left(\ell_{g, x} \in L_{g, x}\right) .\right.
$$

${ }^{2} C(X, \mathrm{U}(1))$ is the $G_{0}$-module consisting of $\mathrm{U}(1)$-valued functions on $X$. The $G_{0}$-structure is defined by $(g \cdot f)(x):=f(g \cdot x), g \in G_{0}, x \in X$. The group cohomology $H^{2}\left(G_{0}, C(X, \mathrm{U}(1))\right)$ classifies the following extension

$$
1 \rightarrow C(X, \mathrm{U}(1)) \rightarrow \hat{G} \rightarrow G_{0} \rightarrow 1
$$




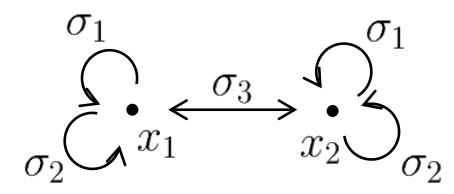

Figure 12. Example of a $G_{0}$-set $X$ for a combination of symmetry broken and symmetry fractionalization. In this example, $X$ consists of two points $X=\left\{x_{1}, x_{2}\right\}$ which are permuted by the broken symmetry $\sigma_{3} \in G_{0}$.

The associativity condition $\ell_{g_{3}, x_{3}}\left(\ell_{g_{2}, x_{2}} \ell_{g_{1}, x_{1}}\right)=\left(\ell_{g_{3}, x_{3}} \ell_{g_{2}, x_{2}}\right) \ell_{g_{1}, x_{1}}$ corresponds to the 2 cocycle condition

$$
b_{g_{1} \cdot x}\left(g_{3}, g_{2}\right) b_{x}\left(g_{3} g_{2}, g_{1}\right)=b_{x}\left(g_{2}, g_{1}\right) b_{x}\left(g_{3}, g_{2} g_{1}\right) .
$$

In short, $G_{0}$-equivariant TFTs are classified by the group cohomology $H^{2}\left(G_{0}, C(X, \mathrm{U}(1))\right)\left(\cong H_{G_{0}}^{2}(X, \mathrm{U}(1))\right)$.

To make a contact with physics of SPT phases, let us specialize to the case where $\mathcal{C}_{1}$ is simple $\mathcal{C}_{1} \cong \mathbb{C}$. In this case, the ground state in the untwisted sector is unique, and the classification is reduced into group cohomology with $\mathrm{U}(1)$ coefficient $H^{2}\left(G_{0}, \mathrm{U}(1)\right)$.

On the other hand, in semisimple cases, we have a combination of symmetry breaking and symmetry fractionalization discussed in refs. [3, 27]. Since the group cohomology $H^{2}\left(G_{0}, C(X, \mathrm{U}(1))\right)$ splits into $G_{0}$-orbits, we can simply assume that $X$ consists of a single $G_{0}$-orbit. Let subgroup $G^{\prime} \subset G_{0}$ be an unbroken symmetries, then, we have a bijection $X \cong G_{0} / G^{\prime}$ as a set, which is a "Nambu-Goldstone manifold". Each element $x \in G_{0} / G^{\prime}$ represents a vacuum which partially breaks $G_{0}$ symmetry and retains $G^{\prime}$ symmetry. All the elements $G_{0} / G^{\prime}$ are permuted by broken symmetries in $G_{0}$. The topological classification is given by

$$
H_{G_{0}}^{2}\left(G_{0} / G^{\prime}, \mathrm{U}(1)\right) \cong H_{G^{\prime}}^{2}(p t, \mathrm{U}(1)) \cong H^{2}\left(G^{\prime}, \mathrm{U}(1)\right),
$$

says, the group cohomology classification for unbroken symmetries.

For example, let full symmetry be $G_{0}=\mathbb{Z}_{2}\left[\sigma_{1}\right] \times \mathbb{Z}_{2}\left[\sigma_{2}\right] \times \mathbb{Z}_{2}\left[\sigma_{3}\right]$ and unbroken symmetry be $G^{\prime}=\mathbb{Z}_{2}\left[\sigma_{1}\right] \times \mathbb{Z}_{2}\left[\sigma_{2}\right]$, where $\sigma_{i}(i=1,2,3)$ are generators of $\mathbb{Z}_{2}$. In this case, $X$ consists of two points $\left\{x_{1}, x_{2}\right\}$ which are exchanged by the broken symmetry as $x_{2}=\sigma_{3} \cdot x_{1}$ as shown in figure 12. The topological classification is given by that for the unbroken symmetry as $H^{2}\left(\mathbb{Z}_{2}\left[\sigma_{1}\right] \times \mathbb{Z}_{2}\left[\sigma_{2}\right], \mathrm{U}(1)\right)=\mathbb{Z}_{2}$. See figure 12 .

\subsection{G-equivariant unoriented closed TFTs}

$(1+1)$ d oriented (closed) TFTs were extended to unoriented TFTs by Turaev-Turner [28] and equivariant unoriented TFTs by Kapsutin-Turzillo [12]. See also refs. [29, 30]. Here we review $G$-equivariant unoriented $(1+1)$ d TFTs.

As before, let $G$ be a full symmetry group including orientation-reversing symmetries and $G_{0} \subset G$ be the orientation-preserving subgroup. There are two new ingredients to define (equivariant) unoriented $(1+1)$ d TFTs: the crosscap state and reflection transformation $((\mathrm{g})$ and $(\mathrm{h})$ in table 1 , respectively). As for Item $(\mathrm{g})$, the boundary state of the 
Möbius strip defines the crosscap state $\theta_{g} \in \mathcal{C}_{g^{2}}\left(g \notin G_{0}\right)$

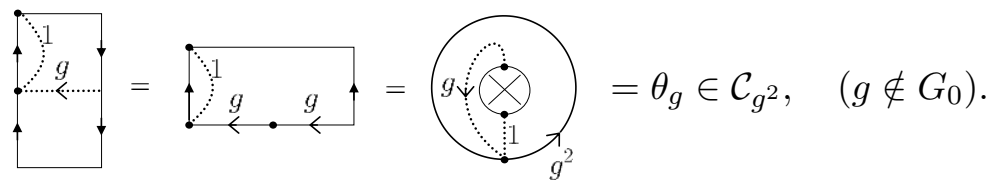

Notice that the crosscap state $\theta_{g}$ belongs to the twisted sector of $g^{2} \in G_{0}$. As for Item (h), the presence of an orientation-reversing symmetry $g \notin G_{0}$ can be used to consider reflection of the circle

$$
\underbrace{}_{g h g^{-1}}
$$

In a way similar to $G_{0}$-equivariant oriented TFTs, we have several constraints on the algebraic category. Kapustin-Turzillo [12] showed that to give a $G$-equivariant unoriented $(1+1) \mathrm{d}$ TFT is equivalent to give a $G_{0}$-graded algebra $\mathcal{C}=\bigoplus_{g \in G_{0}} \mathcal{C}_{g}$ together with a group homomorphism $\alpha: G \rightarrow \operatorname{Aut}(\mathcal{C})$ such that $\alpha_{g \in G_{0}}: \mathcal{C}_{h} \rightarrow \mathcal{C}_{g h g^{-1}}, \alpha_{g \notin G_{0}}: \mathcal{C}_{h} \rightarrow \mathcal{C}_{g h^{-1} g^{-1}}$. They must satisfy (1)-(4), and

(5) $\alpha_{g}\left(\phi_{1} \phi_{2}\right)=\alpha_{g}\left(\phi_{2}\right) \alpha_{g}\left(\phi_{1}\right), g \notin G_{0}$.

(6) $\alpha_{h \in G_{0}}\left(\theta_{g}\right)=\theta_{h g h^{-1}}$ and $\alpha_{h \notin G_{0}}\left(\theta_{g}\right)=\theta_{h g^{-1} h^{-1}}$.

(7) (Punctured Möbius strip) $\theta_{g} \phi=\alpha_{g}(\phi) \theta_{g h}, \phi \in \mathcal{C}_{h}$.

(8) (Punctured Klein bottle) $\sum_{i} \alpha_{g}\left(\xi_{i}^{(g h)^{-1}}\right) \xi_{i}^{g h}=\theta_{g} \theta_{h}, g, h \notin G_{0}$.

Derivations of these constraints [12] are summarized in appendix D.1.

All possible partition functions are constructed from the handle adding operator (3.9) and crosscap adding operator (3.14). For example, the partition function on real projective plane reads

$$
Z_{\mathbb{R} P^{2}}(g)=\bigotimes=\theta_{\mathcal{C}}\left(\theta_{g}\right), \quad\left(g \notin G_{0}, g^{2}=1\right)
$$

The Klein bottle partition function is

$$
Z_{K B}(g ; h)=\theta_{g h g^{-1}}
$$




\subsubsection{General solution for simple cases}

In the cases where $\mathcal{C}_{1}$ is simple $\mathcal{C}_{1} \cong \mathbb{C}$, i.e., there is a unique ground state, KapustinTurzillo [12] showed general solutions of the algebraic constraints (1) - (8). They showed that to give a $G$-equivariant unoriented $(1+1) \mathrm{d}$ simple TFT is to give a 2 -group cycle $b(g, h) \in Z^{2}\left(G, \mathrm{U}(1)_{\phi}\right) .{ }^{3}$ This is consistent with the group cohomology classification of bosonic $(1+1)$ d SPT phases with reflection or time-reversal symmetry [27].

\subsubsection{Relation to MPS}

In the SPT context, the spatial circle $S^{1}$ with $g$-flux in TFTs is identified with a bulk SPT phase with $g$-twisted boundary condition. The uniqueness condition of the ground state in SPT phases implies that the corresponding TFTs are invertible, i.e., we have a simple algebra of untwisted sector $\mathcal{C}_{1} \cong \mathbb{C}$. In TFTs, there is no excited state and the Hilbert space consists only of ground states. The correlation length of the bulk is zero, so a TFT is represented by a fixed point MPS introduced in section 2.3

$$
\left|\Psi_{g}\right\rangle=\sum_{m} \operatorname{Tr}\left(A_{m_{1}} \cdots A_{m_{L}} V_{g}\right)\left|m_{1} \cdots m_{L}\right\rangle \sim \sum_{m} \operatorname{Tr}\left(A_{m} V_{g}\right)|m\rangle
$$

Here we used the equivalence relation of fixed point MPSs (2.25). Only one physical site is sufficient to describe the MPS representation of a TFT's ground state. The correspondence between MPSs and equivariant TFTs, can be pictorially represented as

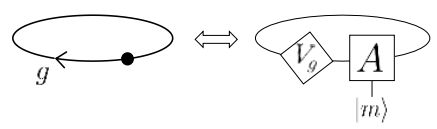

Cobordisms in $G$-equivariant TFTs correspond to various "adiabatic deformations" of closed chains, e.g., "fusion" and "separating", and symmetry operations. The fourth column in table 1 summarizes correspondences between cobordisms in $G$-equivariant TFTs and MPS representations.

For example, the fusion process of two closed chain is formally represented in MPS networks as follows. For two MPSs

$$
\left|\Psi_{g}\right\rangle=\operatorname{Tr}\left[A_{1} V_{g}\right], \quad\left|\Psi_{h}\right\rangle=\operatorname{Tr}\left[A_{2} V_{h}\right]
$$

the fusion $\left|\Psi_{g}\right\rangle \cdot\left|\Psi_{h}\right\rangle$ is given by

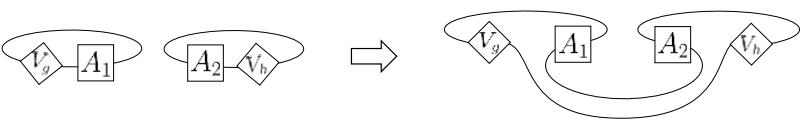

$$
\begin{aligned}
& \Rightarrow\left|\Psi_{g}\right\rangle \cdot\left|\Psi_{h}\right\rangle=\operatorname{Tr}\left[A_{2} A_{1} V_{g} V_{h}\right]\left|m_{2} m_{1}\right\rangle \sim b(g, h) \operatorname{Tr}\left[A V_{g h}\right]|m\rangle=b(g, h)\left|\Psi_{g h}\right\rangle .
\end{aligned}
$$

Here we used the equivalence relation of fixed point MPSs (2.25). 


\begin{tabular}{|c|c|c|c|c|}
\hline & Manifolds & Hilbert spaces & Simple and fixed point MPS & Comment \\
\hline (i) & $b$ & $\mathcal{O}_{a b}$ & $\begin{array}{l}\text { Hilbert space spanned by } \\
\qquad\left\{\left(L_{i}^{T} A_{m} R_{j}\right)|m\rangle\right\} \\
L_{i} \in V_{a}^{*}, R_{j} \in V_{b}\end{array}$ & $\begin{array}{c}\text { Open chain. } L_{i}\left(R_{j}\right) \text { are basis of } \\
V_{a}^{*}\left(V_{b}\right) .\end{array}$ \\
\hline (j) & & $\begin{aligned} \theta_{a} & : \mathcal{O}_{a a} \rightarrow \mathbb{C} \\
\psi & \mapsto \theta_{a}(\psi)\end{aligned}$ & $\begin{array}{c}\theta_{a}\left(\left(v_{L}^{T} A_{m} v_{R}\right)|m\rangle\right)=\left(v_{L}, v_{R}\right), \\
v_{L} \in V_{a}^{*}, v_{R} \in V_{a}\end{array}$ & $\begin{array}{l}\left(v_{L}, v_{R}\right)=\sum_{i}\left[v_{L}\right]_{i}\left[v_{R}\right]_{i} . \text { Notice } \\
\text { that } \theta_{a}\left(1_{a}\right)=\operatorname{dim} V_{a} \text { for simple } \\
\text { and fixed point MPS. }\end{array}$ \\
\hline (k) & & $\begin{aligned} \mathbb{C} & \rightarrow \mathcal{O}_{a a} \\
1 & \mapsto 1_{a}\end{aligned}$ & $\sum_{i}\left(L_{i}^{T} A_{m} R_{i}\right)|m\rangle$ & $\begin{array}{l}1_{a} \text { is the unit satisfying } \\
1_{a} \psi=\psi 1_{b}=\psi, \psi \in \mathcal{O}_{a b} .\end{array}$ \\
\hline (l) & & $\begin{array}{c}\rho_{g \in G_{0}}: \mathcal{O}_{a b} \rightarrow \mathcal{O}_{a b}, \\
\psi \mapsto \rho_{g} \psi\end{array}$ & $\begin{array}{l}\left(v_{L}^{T} A_{m} v_{R}\right)|m\rangle \\
\mapsto\left(v_{L}^{T} V_{g, a}^{\dagger} A_{m} V_{g, b} v_{R}\right)|m\rangle \\
v_{L} \in V_{a}^{*}, v_{R} \in V_{b}\end{array}$ & $\begin{array}{l}g \text {-action on open chain. } V_{g, a} \text { is } \\
\text { representation matrix of } V_{a} \text {. }\end{array}$ \\
\hline (m) & & $\begin{array}{c}\rho_{g \notin G_{0}}: \mathcal{O}_{a b} \rightarrow \mathcal{O}_{a b}, \\
\psi \mapsto \rho_{g} \psi\end{array}$ & $\begin{array}{l}\left(v_{L}^{T} A_{m} v_{R}\right)|m\rangle \\
\mapsto\left(v_{R}^{T} V_{g, b}^{\dagger} A_{m} V_{g, a} v_{L}\right)|m\rangle \\
v_{L} \in V_{a}^{*}, v_{R} \in V_{b}\end{array}$ & $g$-reflection on an open chain \\
\hline (n) & & $\begin{array}{c}\mathcal{O}_{a b} \otimes \mathcal{O}_{b c} \rightarrow \mathcal{O}_{a c} \\
\psi_{1} \otimes \psi_{2} \mapsto \psi_{1} \psi_{2}\end{array}$ & $\begin{array}{c}\left(v_{L}^{T} A_{m_{1}} v_{R}\right)\left|m_{1}\right\rangle \otimes\left(w_{L}^{T} A_{m_{2}} w_{R}\right)\left|m_{2}\right\rangle \\
\mapsto\left(w_{L}, v_{R}\right)\left(v_{L}^{T} A_{m} w_{R}\right)|m\rangle \\
v_{L} \in V_{a}^{*}, v_{R} \in V_{b}, w_{L} \in V_{b}^{*}, w_{R} \in V_{c}\end{array}$ & Fusion of two open chains \\
\hline (o) & & $\begin{aligned} \mathbb{C} & \rightarrow \mathcal{O}_{a b} \otimes \mathcal{O}_{b a} \\
1 & \mapsto \sum_{\mu} \psi_{\mu} \otimes \psi^{\mu}\end{aligned}$ & $\sum_{i j}\left(L_{i}^{T} A_{m_{1}} R_{j}\right)\left|m_{1}\right\rangle \otimes\left(L_{j}^{T} A_{m_{2}} R_{i}\right)\left|m_{2}\right\rangle$ & $\begin{array}{c}\psi_{\mu} \in \mathcal{O}_{a b} \text { are basis of } \mathcal{O}_{a b} \text { and } \\
\psi^{\mu} \in \mathcal{O}_{b a} \text { are their dual of } \mathcal{O}_{b a} \\
\text { that satisfy } \theta_{a}\left(\psi_{\mu} \psi^{\nu}\right)=\delta_{\mu}^{\nu}\end{array}$ \\
\hline (p) & & $\begin{aligned} \imath^{g, a} & : \mathcal{O}_{a a} \rightarrow \mathcal{C}_{g} \\
\psi & \mapsto \imath^{g, a}(\psi)\end{aligned}$ & $\begin{array}{c}\left(v_{L}^{T} A_{m} v_{R}\right)|m\rangle \mapsto \\
\left(V_{g}^{*} v_{L}, v_{R}\right) \operatorname{Tr}\left(A_{m} V_{g}\right)|m\rangle\end{array}$ & Open to closed map \\
\hline (q) & & $\begin{aligned} \imath_{g, a} & : \mathcal{C}_{g} \rightarrow \mathcal{O}_{a a} \\
\quad \phi & \mapsto \imath_{g, a}(\phi)\end{aligned}$ & $\operatorname{Tr}\left(A_{m} V_{g}\right)|m\rangle \mapsto \sum_{i}\left(L_{i}^{T} A_{m} V_{g} R_{i}\right)|m\rangle$ & Closed to open map \\
\hline$(\mathrm{r})$ & & $B_{a}=\imath^{g, a}\left(1_{a}\right) \in \mathcal{C}_{g}$ & $\operatorname{tr}\left(V_{g, a}^{\dagger}\right) \operatorname{Tr}\left(A_{m} V_{g, a}\right)|m\rangle$ & $\begin{array}{l}\text { Boundary state for boundary } \\
\text { condition } a \text {. }\end{array}$ \\
\hline
\end{tabular}

Table 2. Building blocks of $G$-equivariant open and closed $(1+1)$ d TFTs. In the forth column, MPS representations are shown. In figures, dashed lines with group elements represent holonomies. (r) is the definition of the boundary state for a boundary condition $a$. 


\section{$3.4 \quad G$-equivariant open and closed TFTs}

Next, we extend closed TFTs to include open chains (intervals). A new object is an oriented interval $I_{a b}=[0,1]$ with boundary conditions $a, b$ as shown in table $2(\mathrm{i}) .{ }^{4}$ We denote the Hilbert space associated with the interval $I_{a b}$ by $\mathcal{O}_{a b}$. An element $\psi \in \mathcal{O}_{a b}$ represents a state living in the open chain with boundary conditions $a$ and $b$. (Note that the boundary conditions $a, b$ do not represent some states in the open chain. )

Similar to closed TFTs, we have several cobordisms in open and closed TFTs. Table 2 summarizes the building blocks. We have some remarks in order.

- We use the same notation as Moore-Segal [11]. The fusion process is represented as

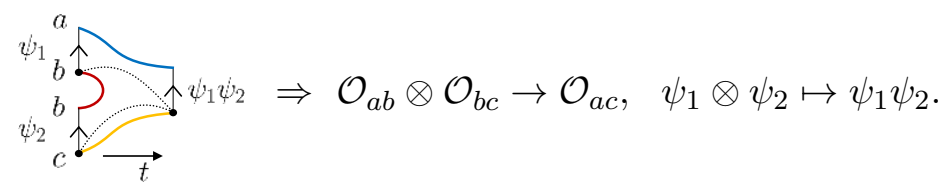

Note the order of two intervals $I_{a b}$ and $I_{b c}$.

- In addition to on-site symmetry transformation $\rho_{g \in G_{0}}: \mathcal{O}_{a b} \rightarrow \mathcal{O}_{a b}$, we have reflection on an open chain $\rho_{g \notin G_{0}}: \mathcal{O}_{a b} \rightarrow \mathcal{O}_{b a}$ which exchanges the boundary conditions $a, b$. $\rho_{g}$ satisfies $\rho_{g} \circ \rho_{h}=\rho_{g h}(g, h \in G)$.

- Essentially new ingredients are the open-to-closed map $\imath^{g, a}$ and the closed-to-open map $\imath_{g, a}$ which connect closed chains and open chains as [11]

$$
\imath^{g, a}: \mathcal{O}_{a a} \rightarrow \mathcal{C}_{g}, \quad \imath_{g, a}: \mathcal{C}_{g} \rightarrow \mathcal{O}_{a a}, \quad\left(g \in G_{0}\right) .
$$

Here, to glue back to a closed chain from a open chain, the boundary conditions should agree.

All bordsims can be constructed by using building blocks listed in table 2. For example, a "branching" process of an open chain is given by the same way as (3.7),

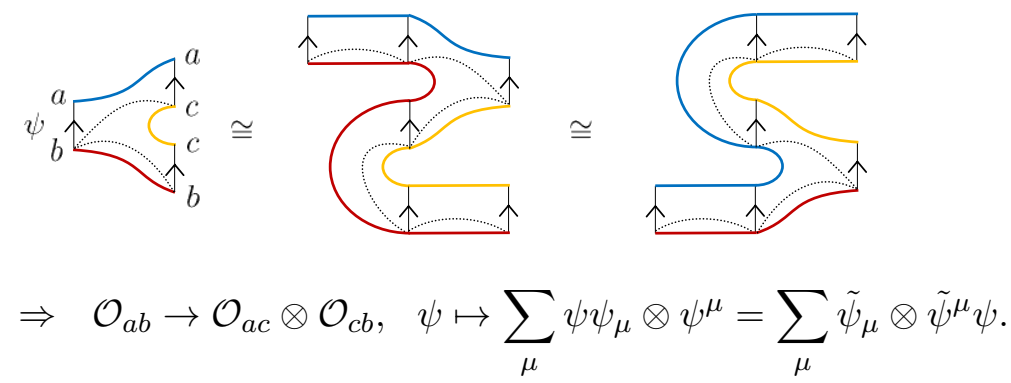

Here, $\sum_{\mu} \psi_{\mu} \otimes \psi^{\mu}\left(\psi_{\mu} \in \mathcal{O}_{b c}, \psi^{\mu} \in \mathcal{O}_{c b}\right)$ and $\sum_{\mu} \tilde{\psi}_{\mu} \otimes \tilde{\psi}^{\mu}\left(\tilde{\psi}_{\mu} \in \mathcal{O}_{a c}, \tilde{\psi}^{\mu} \in \mathcal{O}_{c a}\right)$ are coform defined in (o) of table 2 .

\footnotetext{
${ }^{3} \mathrm{U}(1)_{\phi}$ is equipped with $G$-action defined by (A.1).

${ }^{4}$ In string theory, the boundary conditions $a, b, \ldots$ are Chan-Paton factors associated with the endpoints of open strings.
} 
In the target algebraic category, there are constraints from the open and closed cobordism category. We have the following constraints for oriented open and closed TFTs by Moore-Segal [11]:

(9) $\imath_{1, a}\left(1_{\mathcal{C}}\right)=1_{\mathcal{O}_{a a}}$.

(10) $\rho_{g}\left(\psi_{1} \psi_{2}\right)=\left(\rho_{g} \psi_{1}\right)\left(\rho_{g} \psi_{2}\right), g \in G_{0}, \psi_{1} \in \mathcal{O}_{a b}, \psi_{2} \in \mathcal{O}_{b c}$

(11) $\imath_{g_{1}, a}\left(\phi_{1}\right) \imath_{g_{2}, a}\left(\phi_{2}\right)=\imath_{g_{2} g_{1}, a}\left(\phi_{2} \phi_{1}\right), \phi_{1} \in \mathcal{C}_{g_{1}}, \phi_{2} \in \mathcal{C}_{g_{2}}$.

(12) $\imath_{g, a}(\phi)\left(\rho_{g} \psi\right)=\psi \imath_{g, a}(\phi), \phi \in \mathcal{C}_{g}, \psi \in \mathcal{O}_{a a}$.

(13) $\theta_{a}\left(\psi \imath_{g^{-1}, a}(\phi)\right)=\theta_{\mathcal{C}}\left(\imath^{g, a}(\psi) \phi\right), \phi \in \mathcal{C}_{g^{-1}}, \psi \in \mathcal{O}_{a a}$.

(14) (G-equivariant Cardy condition) $\pi_{g, b}^{a}=\imath_{g, b} \circ \imath^{g, a}$ with $\pi_{g, b}^{a}(\psi)=\sum_{\mu} \psi^{\mu} \psi\left(\rho_{g} \psi_{\mu}\right), g \in$ $G_{0}$.

For unoriented open and closed TFTs, one can find the following additional constraints:

$$
\begin{aligned}
& \text { (15) } \rho_{g}\left(\psi_{1} \psi_{2}\right)=\left(\rho_{g} \psi_{2}\right)\left(\rho_{g} \psi_{1}\right), g \notin G_{0}, \psi_{1} \in \mathcal{O}_{a b}, \psi_{2} \in \mathcal{O}_{b c} . \\
& \text { (16) } \sum_{\mu}\left(\rho_{g} \psi_{\mu}\right) \psi^{\mu}=\imath_{g^{2}, a}\left(\theta_{g}\right), \quad g \notin G_{0}, \psi_{\mu}, \psi^{\mu} \in \mathcal{O}_{a a} .
\end{aligned}
$$

In appendix D.2, we summarize the derivations of these constraints.

By solving these constraints, we can determine the general properties of the target algebraic category for a given $G$-equivariant closed TFT $b \in Z_{G}^{2}\left(X, \mathrm{U}(1)_{\phi}\right)$ with $G$-set $X$. In the cases where $\mathcal{C}_{1}$ is semisimple, i.e., combination of symmetry breaking and symmetry fractionalization, and there is no orientation-reversing symmetry, Moore-Segal [11] gives the complete solution: $b$-twisted equivariant vector bundles over $X$. Here, for simplicity, we assume the ground state of closed chain is unique, $\mathcal{C}_{1} \cong \mathbb{C}$, and there are only on-site symmetries $G_{0}$. We have [11]

- The category of boundary conditions $\{a, b, \ldots\}$ is equivalent to the category of $b$ projective representations $\left\{V_{a}, V_{b}, \ldots\right\}$.

- $\mathcal{O}_{a b} \cong \operatorname{Hom}\left(V_{b}^{*}, V_{a}^{*}\right)=V_{a}^{*} \otimes V_{b}$.

This is precisely the boundary degrees of freedom that appear when one introduce a boundary in SPT phases. In the next section, we describe how to represent elements of $\mathcal{O}_{a b}$ and cobordisms by using simple and fixed point MPS for open chains.

\subsubsection{Relation to open MPS}

In the SPT context, an interval $I_{a b}$ is identified with an open SPT phase with boundary condition $a$ and $b$. An element of $\psi \in \mathcal{O}_{a b} \cong V_{a}^{*} \otimes V_{b}$ is identified with a state of the open chain Hilbert space ${ }^{5}$

$$
\psi=\sum_{m}\left(v_{L}^{T} A_{m} v_{R}\right)|m\rangle=\sum_{m}\left[v_{L}\right]_{i}\left[A_{m}\right]_{i j}\left[v_{R}\right]_{j}|m\rangle, \quad v_{L} \in V_{a}^{*}, \quad v_{R} \in V_{b} .
$$

\footnotetext{
${ }^{5}$ Note that $a, b$ do not specify a state in the representation space of the $b(g, h)$-projective representations. For example, the dihedral group $D_{4}=\left\{1, C_{4}, C_{2}, C_{4}^{-1}, \sigma_{x}, \sigma_{y}, \sigma_{d}, \sigma_{d}^{\prime}\right\}$ has two $b$-irreps $E_{\frac{1}{2}}$ and $E_{\frac{3}{2}}$ for the nontrivial two cocycle $[b] \in H^{2}\left(D_{4}, \mathrm{U}(1)\right) \cong \mathbb{Z}_{2}$. In this case, $a, b$ specify $E_{\frac{1}{2}}$ or $E_{\frac{3}{2}}$.
} 
The correspondence between MPSs and equivariant TFTs, can be pictorially represented as

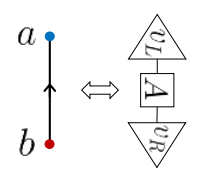

Cobordisms in $G$-equivariant open and closed TFTs correspond to various "adiabatic deformations" of open chains and closed chains. The fourth column in table 2 summarizes MPS representations, which satisfy algebraic constraints (9) - (16).

For example, the fusion process of two open chains is represented in MPS networks as follows. For two open MPSs

$$
\begin{array}{ll}
\psi_{1}=\sum_{m} v_{L}^{T} A_{m}^{(1)} v_{R}|m\rangle, & v_{L} \in V_{a}^{*}, v_{R} \in V_{b}, \\
\psi_{2}=\sum_{m} w_{L}^{T} A_{m}^{(2)} w_{R}|m\rangle, & w_{L} \in V_{b}^{*}, w_{R} \in V_{c},
\end{array}
$$

the fusion $\psi_{1} \psi_{2}$ is given by

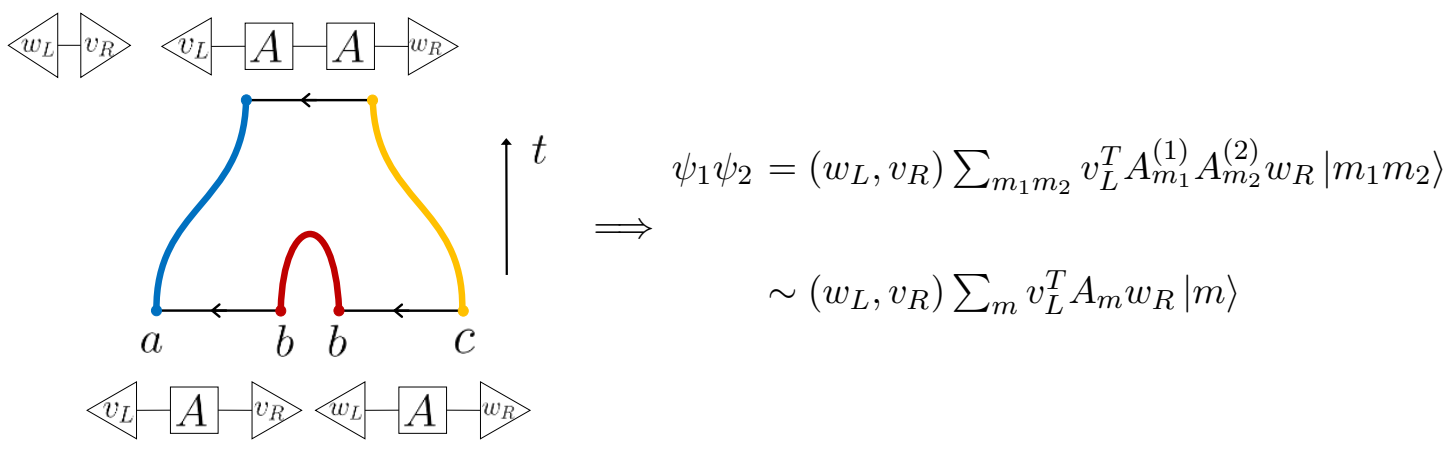

Here we introduced a notation $\left(w_{L}, v_{R}\right)=\sum_{i}\left[w_{L}\right]_{i}\left[v_{R}\right]_{i}$ and used an equivalence relation of fixed point MPSs (2.25).

\subsubsection{Equivariant Cardy conditions and boundary states}

In the derivation of the category of boundary conditions by Moore-Segal [11], the (generalized) $G$-equivariant Cardy condition (14) plays an essential role. Here, we show MPS representations listed in the fourth column in table 2 satisfy the $G$-equivariant Cardy condition. The Cardy condition comes from the equivalence between (i) the double twist diagram shown in the left of figure 13 and (ii) closed string channel shown in the right of figure 13. These diagram can be interpreted in the context of opne SPT chains:

(i) Cutting an open SPT chain $I_{a a}$ into two open chains $I_{a b} \sqcup I_{b a}$ and taking the $G_{0^{-}}$ action on the left chain $\mathcal{O}_{a b}$ and exchanging two open chains and gluing back at $a$. 


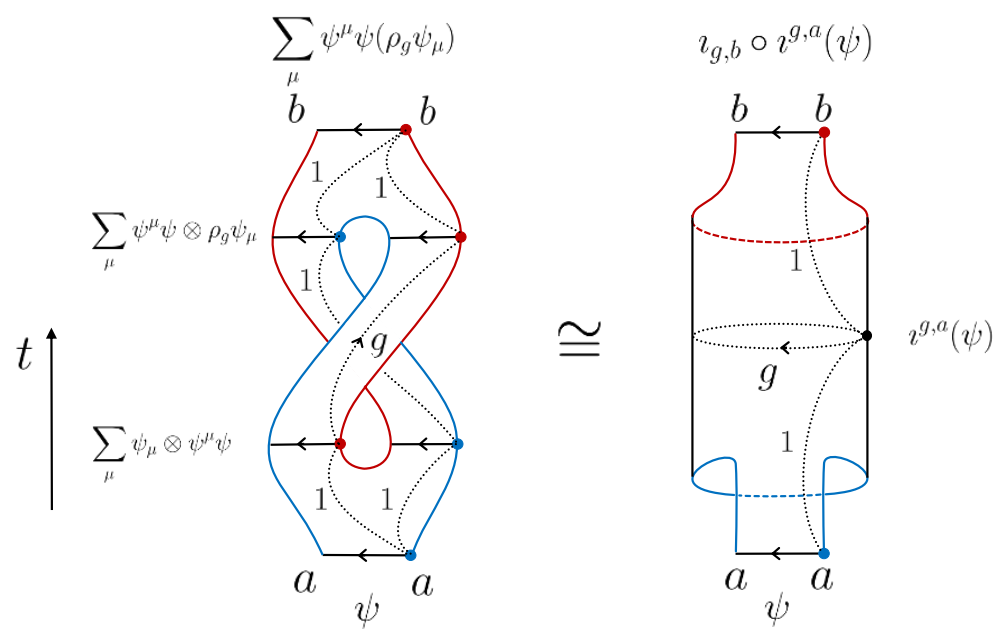

Figure 13. G-equivariant Cardy condition.

This process is written as

$$
\begin{aligned}
\psi=\left(v_{L}^{T} A_{m} v_{R}\right)|m\rangle & \mapsto \sum_{j}\left(v_{L}^{T} A_{m_{1}} R_{j}\right)\left|m_{1}\right\rangle \otimes\left(L_{j}^{T} A_{m_{2}} v_{R}\right)\left|m_{2}\right\rangle \\
& \mapsto \sum_{j}\left(v_{L}^{T} V_{g}^{\dagger} A_{m_{1}} V_{g} R_{j}\right)\left|m_{1}\right\rangle \otimes\left(L_{j}^{T} A_{m_{2}} v_{R}\right)\left|m_{2}\right\rangle \\
& \mapsto \sum_{j}\left(L_{j}^{T} A_{m_{2}} v_{R}\right)\left|m_{2}\right\rangle \otimes\left(v_{L}^{T} V_{g}^{\dagger} A_{m_{1}} V_{g} R_{j}\right)\left|m_{1}\right\rangle \\
& \mapsto\left(V_{g}^{*} v_{L}, v_{R}\right) \sum_{j}\left(L_{j}^{T} A_{m} R_{j}\right)|m\rangle .
\end{aligned}
$$

(ii) Gluing the both ends of open SPT chain $I_{a a}$ to the $g$-twisted closed chain $\left(S^{1}, g\right)$ and cutting into the open chain $I_{b b}$. This process is expressed as

$$
\psi=\left(v_{L}^{T} A_{m} v_{R}\right)|m\rangle \mapsto\left(V_{g}^{*} v_{L}, v_{R}\right) \operatorname{tr}\left(A_{m} V_{g}\right)|m\rangle \mapsto\left(V_{g}^{*} v_{L}, v_{R}\right) \sum_{j}\left(L_{j}^{T} A_{m} R_{j}\right)|m\rangle .
$$

It is useful to introduce the equivariant boundary state $B_{g, a} \in \mathcal{C}_{g}$ in a way similar to usual boundary state $B_{a}$ for non-equivariant TFTs. Defining property of boundary state is that the correlation functions on upper half plane with boundary condition $a$ (figure 14 [a]) is the same as the closed string amplitude with insertion of the boundary state (figure $14[\mathrm{~b}]$ ):

$$
\theta_{a}\left(\imath^{h, a}\left(\phi_{1} \phi_{2} \cdots \phi_{n}\right)\right)=\theta_{\mathcal{C}}\left(B_{h^{-1}, a} \phi_{1} \phi_{2} \cdots \phi_{n}\right), \quad \phi_{i} \in \mathcal{C}_{g_{i}}, h=g_{1} g_{2} \cdots g_{n} .
$$

From the algebraic constraint (13), the $G$-equivariant boundary state is given by the image of open to closed map on the unit element $1_{a}$ of the open chain $\mathcal{O}_{a a}$,

$$
B_{g, a}:=\bigcap_{g}^{a}=\imath^{g, a}\left(1_{a}\right) \in \mathcal{C}_{g} .
$$


[a]

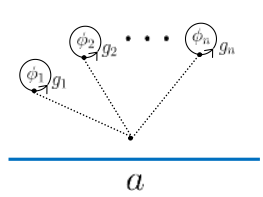

[b]

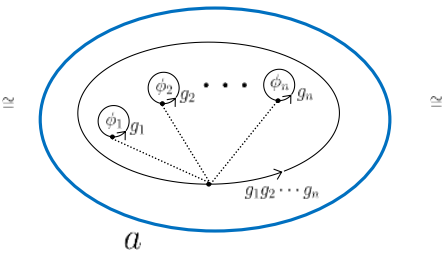

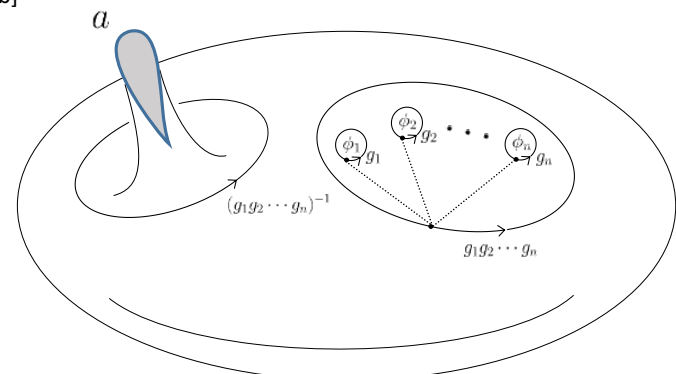

Figure 14. The definition of $G$-equivariant boundary state $B_{g, a}$. [a] Correlation functions on the upper half plane with boundary condition $a$. [b] Amplitude of closed sector with insertion of the boundary state.

For simple and fixed point MPSs, we have

$$
B_{g, a}=\operatorname{tr}\left(V_{g, a}^{\dagger}\right) \operatorname{Tr}\left(A_{m} V_{g, a}\right)|m\rangle,
$$

where $V_{g, a}$ is the representation matrix of the $V_{a}$ representation. Notice that $\chi_{a}(g)^{*}=$ $\operatorname{tr}\left(V_{g, a}^{\dagger}\right)$ is the character of $V_{a}$ representation, which is vacuous if there is a group element element $h \in G_{0},[g, h]=0$ with nontrivial discrete torsion phase $b(g, h) \neq b(h, g)$. [31]

If we insert the boundary states in the Cardy condition (14), we get a more familiar form

$$
\left\langle B_{g, b} \mid B_{g, a}\right\rangle=\theta_{\mathcal{C}}\left(B_{g^{-1}, b} B_{g, a}\right)=\theta_{b}\left(\imath_{g, b} \circ \imath^{g, a}\left(1_{a}\right)\right)=\theta_{b}\left(\pi_{g, b}^{a}\left(1_{a}\right)\right)=\operatorname{Tr}_{\mathcal{O}_{a b}}\left(\rho_{g}\right),
$$

which is the character of $G$-action on the open chain Hilbert space $\mathcal{O}_{a b}$,

$$
\chi_{\mathcal{O}_{a b}}(g)=\operatorname{Tr}_{\mathcal{O}_{a b}}\left(\rho_{g}\right)=\chi_{a}(g)^{*} \chi_{b}(g) .
$$

\subsubsection{Crosscap invariant in open chain}

The partition function on $\mathbb{R} P^{2}, Z_{\mathbb{R} P^{2}}(g)=\theta(g), g \notin G_{0}, g^{2}=1$, can be detected in open chains. Making use of the algebraic relation (16), one can find the Möbius strip with boundary condition $a$ is equivalent to the closed string amplitude from crosscap $\theta_{g}$ to boundary state $B_{g^{2}, a}$,

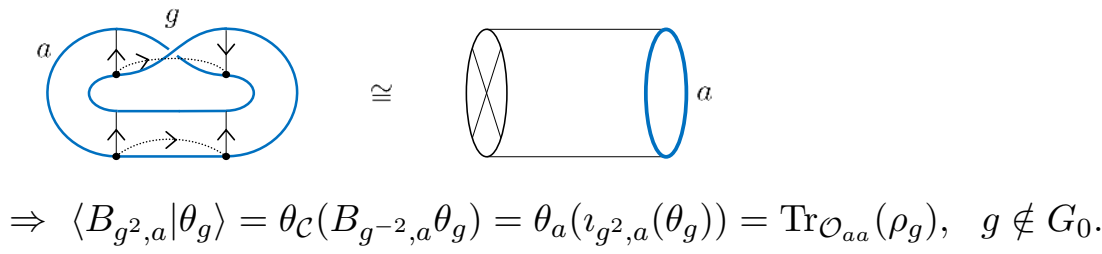

For $g^{2}=1$ and unique ground state $\mathcal{C}_{1} \cong \mathbb{C}$, we have the topological invariant on the real projective plane, which can be confirmed in simple and fixed point MPS as

$$
\theta_{a}\left(\sum_{i j}\left(R_{j}^{T} V_{g}^{\dagger} A_{m_{1}} V_{g} L_{i}\right)\left|m_{1}\right\rangle \cdot\left(L_{j}^{T} A_{m_{2}} R_{i}\right)\left|m_{2}\right\rangle\right)=\left[V_{g}\right]_{j i}\left[V_{g}^{\dagger}\right]_{i j}=\operatorname{dim}\left(V_{a}\right) \theta(g) .
$$




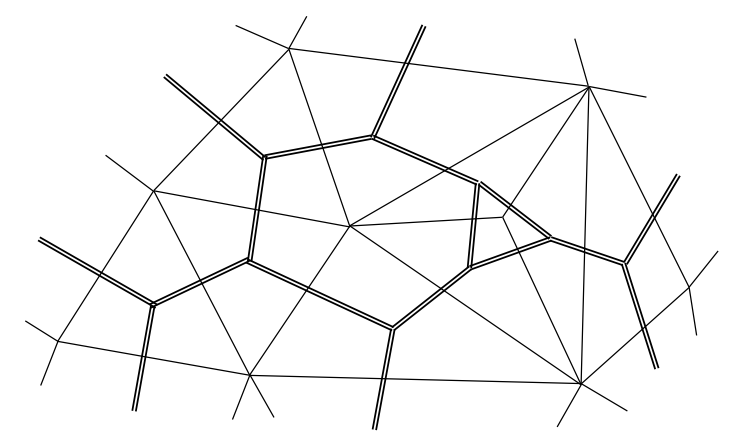

Figure 15. Triangulation of two-dimensional spacetime and its dual (represented by double lines).

\subsection{State sum construction}

In this section, we discuss the so-called state sum construction of TFTs. Compared with the axiomatic approaches discussed previously, the state sum construction exploits specific discretizations (triangulations) of spacetime. We will first review this construction for standard (non-equivariant) TFTs following Fukuma-Hosono-Kawai [14]. We will then consider the state sum construction of $G$-equivariant TFTs, and compute, among others, the partition functions on the torus, Klein bottle, and real projective plane. As promised earlier, we will confirm that they match precisely with the topological (SPT) invariants derived from MPSs.

\subsubsection{Fukuma-Hosono-Kawai state sum construction}

Let us start by briefly reviewing the Fukuma-Hosono-Kawai state sum construction. [14] In the state sum construction of oriented $2 \mathrm{~d}$ TFTs, we consider a triangulation of $2 \mathrm{~d}$ spacetime. For a given triangulation, we can consider its dual, the dual triangulation see figure 15. For faces and edges of the triangulation, we associate $\mathbb{C}$ numbers $C_{\mu \nu \rho}$ and $g^{\mu \nu}(\mu, \nu, \rho=1, \ldots, N)$ as

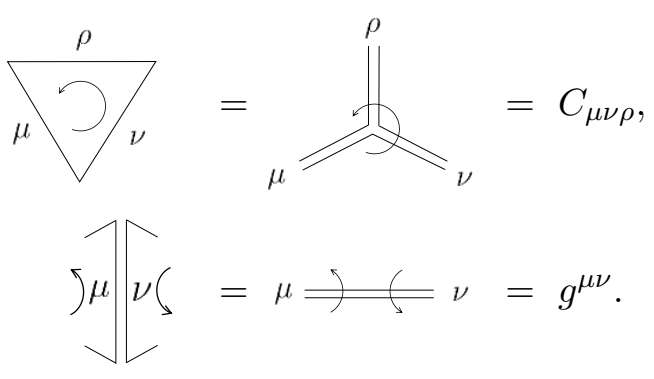

We demand that $C_{\mu \nu \rho}$ is cyclically symmetric $C_{\mu \nu \rho}=C_{\nu \rho \mu}=C_{\rho \mu \nu}$, and $g^{\mu \nu}$ is symmetric $g^{\mu \nu}=g^{\nu \mu} . g_{\mu \nu}$ is defined as the inverse of $g^{\mu \nu}, g^{\mu \nu} g_{\nu \rho}=\delta_{\rho}^{\mu} . g_{\mu \nu}$ and $g^{\mu \nu}$ are used for raising and lowering indices. For example, we introduce $C_{\mu \nu}^{\rho}=C_{\mu \nu \sigma} g^{\sigma \rho}$. For a given triangulation $\Sigma_{T}$ of a surface $\Sigma$, the partition function on $\Sigma_{T}$ is given by

$$
Z\left(\Sigma_{T}\right)=\sum_{\text {faces }} C_{\mu \nu \rho} \sum_{\text {edges }} g^{\eta \epsilon}
$$


In order to make $Z\left(\Sigma_{T}\right)$ independent of triangulations, $C$ and $g$ have to satisfy the fusion and bubble conditions

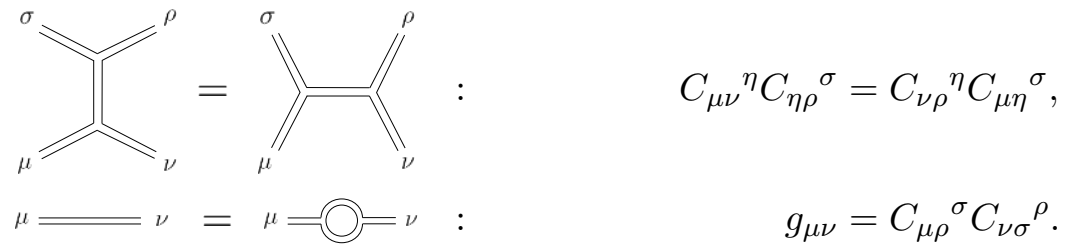

From the data of $C_{\mu \nu \rho}$ and $g^{\mu \nu}$, one can introduce an algebra $\mathcal{C}=\oplus_{\mu=1}^{N} \mathbb{C} \phi_{\mu}$ as

$$
\phi_{\mu} \phi_{\nu}=C_{\mu \nu \rho} \phi_{\rho}
$$

The fusion condition (3.43) means $\mathcal{C}$ is associative $\left(\phi_{\mu} \phi_{\nu}\right) \phi_{\rho}=\phi_{\mu}\left(\phi_{\nu} \phi_{\rho}\right)$. We define a bilinear form by $Q\left(\phi_{\mu}, \phi_{\nu}\right)=g_{\mu \nu}$. Existence of inverse of $g_{\mu \nu}$ ensures that $Q$ is nondegenerate and the algebra $\mathcal{C}$ is semi simple. The cyclicity condition of $C_{\mu \nu \rho}$ leads to the Frobenius condition $Q\left(\phi_{\mu} \phi_{\nu}, \phi_{\rho}\right)=Q\left(\phi_{\mu}, \phi_{\nu} \phi_{\rho}\right)$, i.e., $\mathcal{C}$ is a semi simple Frobenius algebra.

One can show that all the physical observables constructed from the data $C_{\mu \nu \rho}$ and $g^{\mu \nu}$ depend only on the center of $\mathcal{C}, Z(\mathcal{C})=\left\{\phi \in \mathcal{C} \mid \phi \phi^{\prime}=\phi^{\prime} \phi, \forall \phi^{\prime} \in \mathcal{C}\right\}$. [14] In other words, the Fukuma-Hosono-Kawai state sum construction describes $2 \mathrm{~d}$ oriented TFTs which are equivariant to commutative semisimple Frobenius algebras. For example, for a matrix algebra $\mathcal{C}=\operatorname{Mat}\left(\mathbb{C}^{N}\right)$ with $Q(A, B):=\operatorname{Tr} A B$, the center is trivial: $Z\left(\operatorname{Mat}\left(\mathbb{C}^{N}\right)\right)=\mathbb{C} 1_{N \times N}$.

\subsection{2 $G$-equivariant state sum construction}

The state sum construction of $G$-equivariant closed TFTs (both oriented and unoriented) can be formulated in a way analogous to the Fukuma-Hosono-Kawai construction of $2 \mathrm{~d}$ oriented TFTs. [8] In the following, we will discuss this within the context of TFTs describing SPT phases.

As before, let $G$ be a symmetry group which possibly includes orientation-reversing symmetries. We specify orientation-preserving elements by subgroup $G_{0} \subset G$. We fix a group 2-cocycle $b(g, h) \in Z^{2}\left(G, \mathrm{U}(1)_{\phi}\right)$ and assume $[b(g, h)] \in H^{2}\left(G, \mathrm{U}(1)_{\phi}\right)$ is nontrivial. Let $V$ be a $b$-projective $N$-dimensional irrep. and $V^{*}$ be its dual. Recall that $V$ represents the "bond Hilbert space" in MPSs. $V$ also play an analogous role in the state sum construction, which will be developed in the following. The $G$ symmetry is projectively represented in the bond Hilbert space as

$$
\hat{g}(|i\rangle)=|j\rangle\left[V_{g}\right]_{j i}, \quad V_{g} V_{h}=b(g, h) V_{g h}, \quad|i\rangle,|j\rangle \in V,
$$

in the same way as section 2.3.

As in the Fukuma-Hosono-Kawai construction, we need the input data - the Frobenius algebra - to boot-strap a $G$-equivariant TFT. To describe SPT phases (i.e., invertible TFTs), we take the matrix algebra of $V$ as the algebra $\mathcal{C}, \mathcal{C}:=\operatorname{End}(V) \cong V \otimes V^{*}$. The bilinear non-degenerate form is defined by the matrix trace $Q(X, Y)=N \operatorname{tr}(X Y)=$ $N \sum_{i j} X_{i j} Y_{j i}$. 
A canonical basis of $\mathcal{C}$ can be given as

$$
\left\{E_{i j}=|i\rangle\langle j|\right\}
$$

In this basis, $C_{i j, k l, m n}=C\left(E_{i j}, E_{k l}, E_{m n}\right)$ and $g=g^{i j, k l} E_{i j} \otimes E_{k l}$ are given by

$$
\begin{gathered}
C_{i j, k l, m n}=N \\
g^{i j, k l}=\frac{1}{N} \frac{\left.\prod_{i}^{j}\right)\left({ }_{l}^{k}\right.}{l}=\frac{1}{N} \delta^{i l} \delta^{k j} .
\end{gathered}
$$

One can show $Q\left(E_{i j}, E_{k l}\right)=N g_{i j, k l}=N \delta_{i l} \delta_{k j}, C_{i j, k l}{ }^{m n}=\delta_{k j} \delta_{i}^{m} \delta_{l}^{n}, E_{i j} E_{k l}=C_{i j, k l}{ }^{m n} E_{m n}$, and can check (3.43), and (3.44).

Form the construction, the algebra $\mathcal{C}$ has $G$ action

$$
\begin{array}{ll}
\hat{g}(X)=V_{g} X V_{g}^{\dagger}, & X \in \mathcal{C}, g \in G_{0}, \\
\hat{P}(X)=V_{P} X^{T} V_{P}^{\dagger}, & X \in \mathcal{C}, P \notin G_{0} .
\end{array}
$$

Here, observe that the orientation-reversing symmetry $P \notin G_{0}$ exchanges left and right. This $G$ action can be used to to incorporate the background $G_{0}$ gauge field in the networks of the state sum construction. We introduce a symmetry twisted metric by

$$
\left[T_{g}\right]^{i j, k l}:=g^{i j, p q} Q\left(E_{p q}, \hat{g}\left(E_{r s}\right)\right) g^{r s, k l}=\frac{1}{N}\left[V_{g}\right]_{i l}\left[V_{g}^{\dagger}\right]_{k j}=\overbrace{\bar{V}_{g}} \overbrace{V_{l}}^{j}\left(g \in G_{0}\right)
$$

We replace $g^{i j, k l}$ by $\left[T_{g}\right]^{i j, k l}$ on a nontrivial 1-cycle of the triangulation. On the other hand, an orientation reversing symmetry $g \notin G_{0}$ induces the exchange of indices $i$ and $j$. We introduce the orientation reversing twisted metric [15] by

$$
\begin{aligned}
& {\left[T_{P}\right]^{i j, k l}:=g^{i j, p q} Q\left(E_{p q}, \hat{P}\left(E_{r s}\right)\right) g^{r s, k l}=\frac{1}{N}\left[V_{P}\right]_{i k}\left[V_{P}^{\dagger}\right]_{l j}=} \\
& \quad\left(P \notin G_{0}\right) .
\end{aligned}
$$

\subsubsection{Partition functions}

Let us now construct, by using the state sum, the partition functions on $T^{2}$, the Klein bottle, and $\mathbb{R} P^{2}$ (with symmetry twist). We will show that these match precisely with the topological invariants discussed and constructed by using MPSs in section 2.4.

Partition function on $\boldsymbol{T}^{\mathbf{2}}$ with twist. A background $G_{0}$ gauge field on a torus $T^{2}$ is specified by two commuting elements $g, h \in G_{0},[g, h]=0, g, h \in G_{0}$. From the twisted 
metrics $T_{g}, T_{h}$ we have the torus partition function with twist

$$
\begin{aligned}
Z_{T^{2}}(g, h) & =C_{\text {Th }}=C_{i j, k l}{ }^{m n} C_{m n, p q, r s}\left[T_{g}\right]^{i j, p q}\left[T_{h}\right]^{r s, k l} \\
& =\frac{1}{N} \operatorname{tr}\left(V_{g} V_{h} V_{g}^{\dagger} V_{h}^{\dagger}\right)=\epsilon(g, h), \quad\left(g, h \in G_{0}, h g h^{-1}=g\right) .
\end{aligned}
$$

This is the discrete torsion phase (2.26), a topological invariant that characterizes $H^{2}\left(G, \mathrm{U}(1)_{\phi}\right)$.

Partition function on the Klein bottle with twist. Similar to the torus partition function with twist, the Klein bottle partition function with twist is computed in the state sum construction. Let $P \notin G_{0}$ be an orientation reversing symmetry and $g \in G_{0}$ be a orientation preserving symmetry. We have

$$
\begin{aligned}
Z_{K B}(P ; g) & = \\
& =\frac{1}{N} \operatorname{tr}\left(V_{P} V_{g}^{T} V_{P}^{\dagger} V_{g}^{\dagger}\right)=\kappa(P ; g), \quad\left(g \in G_{0}, P \notin G_{0}, P g^{-1} P^{-1}=g\right) .
\end{aligned}
$$

Here, $\kappa(P ; g)$ is the Klein bottle invariant of $H^{2}\left(G, \mathrm{U}(1)_{\phi}\right)$ introduced in (2.28).

Partition function on $\mathbb{R} \boldsymbol{P}^{\mathbf{2}}$. By using the orientation-reversing symmetry $P \in G_{0}$, we can construct the partition funciton on the real projective plane $\mathbb{R} P^{2}$ as

$$
\begin{aligned}
Z_{\mathbb{R} P^{2}}(P) & =C_{i j, k l}{ }^{m n} C_{m n, p q, r s}\left[T_{P}\right]^{i j, p q}\left[T_{P}\right]^{r s, k l} \\
& =\frac{1}{N^{2}} \operatorname{tr}\left(V_{P} V_{P}^{*}\right) \operatorname{tr}\left(V_{P} V_{P}^{\dagger}\right)=\theta(P), \quad\left(P \notin G_{0}, P^{2}=1\right) .
\end{aligned}
$$

This is the cross cap invariant (2.27).

\subsubsection{Cobordisms}

In addition to the closed surfaces considered above, we can also consider surfaces with boundaries by using the state sum construction. From the generalities of TFTs, a surface with boundary represents a state of the Hilbert space. Here, we will construct various states that can be obtained by considering state sum with open boundary/boundaries. For our TFTs that describe SPT phases, the physical Hilbert space $\mathcal{C}$ is spanned by a basis of algebra $\left\{E_{i j}\right\}_{i, j=1}^{N}$. 
Disc (cap state). By the path integral on the disc, we define a state associated to the disc (the cap state). The cap state is the vacuum state on untwisted sector. By triangulating the disc, the path-integral can be evaluated explicitly as

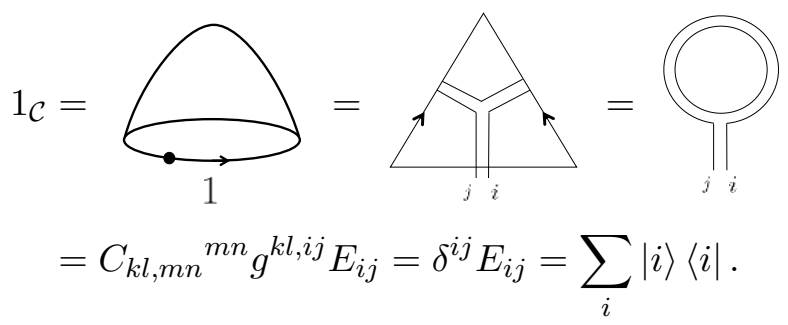

This is nothing but the simple and fixed point MPS representation of the ground state of SPT phases $\left|\Psi_{1}\right\rangle=\operatorname{tr}\left(A_{i j}\right)\left|i^{L}\right\rangle \otimes\left|j^{R}\right\rangle=\frac{1}{\sqrt{N}} \sum_{i}\left|i^{L}\right\rangle \otimes\left|i^{R}\right\rangle$ introduced in section 2.3 up to a normalization.

Möbius strip (cross cap state). By the path integral on the Möbius strip, we define a state associated to the Möbius strip (the cross cap state). By triangulating the Möbius strip, the path-integral can be evaluated explicitly as

$$
\begin{aligned}
\theta_{P} & =\underbrace{}_{P^{2}}=C_{m n, p q}{ }^{i j}\left[T_{P}\right]^{m n, p q} E_{i j}=\frac{1}{N}\left[V_{P} V_{P}^{*}\right]_{i j} E_{i j}=\frac{1}{N} b(P, P) \sum_{i j}\left[V_{P^{2}}\right]_{i j}|i\rangle\langle j|, \quad\left(P \notin G_{0}\right) .
\end{aligned}
$$

Coform $\boldsymbol{\Delta}_{g}$. For a cylinder with two outgoing circles, following the axiom of TFTs, we associate a coform $\Delta_{g}$. By triangulating the cylinder, we have

$$
\begin{aligned}
\Delta_{g} & ={ }_{\xi_{i}^{g}}^{i j}{ }_{m n, p q} C^{k l, m n}{ }_{r s}\left[T_{g}\right]^{p q, r s} E_{i j} \otimes E_{k l} \\
& =\frac{1}{N^{2}} \sum_{i j k l}\left[V_{g}^{\dagger}\right]_{i j}\left[V_{g}\right]_{k l} E_{i j} \otimes E_{k l}, \quad\left(g \in G_{0}\right) .
\end{aligned}
$$

From this, we can read off the twisted ground state $\ell_{g}$ as

$$
\ell_{g}=\frac{1}{N} \sum_{i j}\left[V_{g}\right]_{i j}|i\rangle\langle j| .
$$

This is the same as the fixed point MPS ground state with twist up to a normalization. 
Cylinder $\boldsymbol{\alpha}_{\boldsymbol{g}}$. Since the Hilbert space with twist $\mathcal{C}_{h}\left(h \in G_{0}\right)$ is defined on the circle with $h$-flux, we have to associate cylinder with twist by $T_{h}$. For orientation preserving action $g \in G_{0}, \alpha_{g}$ reads

$$
\begin{aligned}
& \left.\alpha_{g \in G_{0}}\right|_{\mathcal{C}_{h}}=\underbrace{h}_{g h g^{-1}}=\underbrace{h}_{l k} \\
& =C_{i j, m n}{ }^{p q} C_{p q, r s, t u}\left[T_{h}\right]^{t u, m n}\left[T_{g}\right]^{k l, r s} E^{i j} \otimes E_{k l} \\
& =\frac{1}{N}\left[V_{h}^{*}\right]_{i j} E^{i j} \otimes\left[V_{g} V_{h} V_{g}^{\dagger}\right]_{k l} E_{k l}, \quad\left(g \in G_{0}\right) \text {. }
\end{aligned}
$$

Here, $E^{i j}$ is dual basis of $E_{i j}$.

For an orientation reversing action $g \notin G_{0}, \alpha_{g}$ reads

$$
\begin{aligned}
\left.\alpha_{g \notin G_{0}}\right|_{\mathcal{C}_{h}} & = \\
& =C_{i j, m n^{p q}} C_{p q, r s, t u}\left[T_{h}\right]^{t u, m n}\left[T_{P}\right]^{k l, r s} E^{i j} \otimes E_{k l} \\
& =\frac{1}{N}\left[V_{h}^{*}\right]_{i j} E^{i j} \otimes\left[V_{P} V_{h}^{T} V_{P}^{\dagger}\right]_{k l} E_{k l}, \quad\left(g \in G_{0}\right) .
\end{aligned}
$$

Fusion. The sphere with three punctures (the pants diagram) describes a fusion process $\mathcal{C}_{g} \otimes \mathcal{C}_{h} \rightarrow \mathcal{C}_{g h}\left(g, h \in G_{0}\right)$. The path integral can be evaluated as

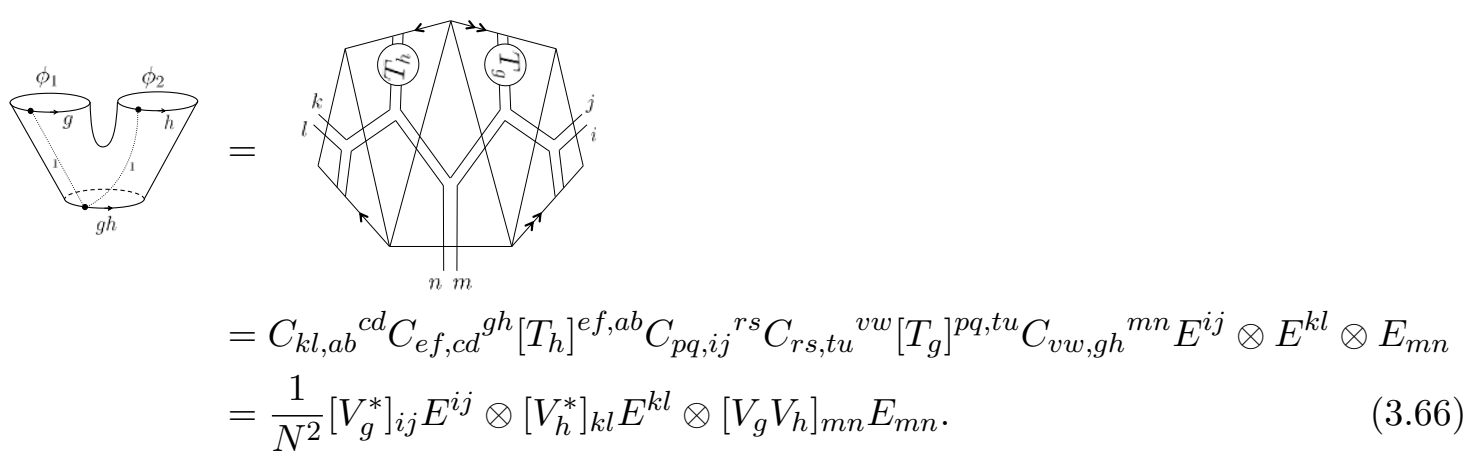

\section{Conclusion}

Tensor networks methods have have been employed as an efficient way to represent correlated, entangled, many-body ground states. In particular, they are expected to provide a powerful framework to study gapped quantum many-body systems with (symmetryprotected) topological order. On the other hand, topological quantum field theories have 
been playing an important guiding role in topological phases of matter. Indeed, one definition of a topological phase of matter is simply that it is described by a TFT. These two descriptions (methods) are complementary to each other: the tensor network methods in general can provide a powerful practical (numerical) framework to study a given lattice model. Within the tensor network framework, it is important to develop a methodology to diagnose topological properties of a given (ground state) many-body wave function. E.g., to develop a method to extract topological invariants from a given many-body wave function (in the tensor network representation). On the other hand, TFTs allow us to work directly in the topological limit (the limit of zero-correlation length), and hence provide a systematic and abstract (axiomatic) framework to, e.g., systematically classify possible topological phases of matter.

In this paper, focusing on $(1+1) d$ bosonic SPT phases, we bridge MPTs and TFTs in $(1+1)$ d. In particular, we discuss $(1+1) d$ d $G$-equivariant (possibly unoriented) TFTs, which are TFTs coupled with a background gauge field.

Our results are briefly summarized as follows:

- In section 2, we summarized the construction of SPT invariants in terms of MPS networks. [5] By expressing those by a reduced density matrix, MPS networks representing SPT topological invariants can be identified with path integrals on manifold with a twist. We showed the partial inversion and the adjacent partial transpose leads to partition function on the real projective plane.

- In section 3 we reviewed $(1+1) \mathrm{d} G$-equivariant open and closed TFTs by MooreSegal [11] which, in addition to closed chains in $(1+1) d$ closed TFTs, have $(1+0) d$ open chains as an object. We established a fixed point MPS representation of $(1+$ 1)d $G$-equivariant open and closed TFTs (when TFTs are invertible). A concrete connection between the MPS and TFT descriptions is summarized in table 1 and 2 . In particular, we noted, for example, that the classification of the $G$-equivariant closed unoriented simple TFTs is given by the second group cohomology, which precisely is the known classification of $(1+1)$ d SPT phases (without orientation-reversing symmetry). We also noted that semisimple TFTs correspond to a combination of symmetry breaking and symmetry fractionalization discussed in the MPS context. Furthermore, for $G$-equivariant open TFTs, the category of boundary conditions is equivalent to the known boundary degrees of freedom in $(1+1) \mathrm{d}$ SPT phases.

- In section 3.5, we presented a state sum construction for $G$-equivariant unoriented closed TFT for $(1+1) d$ bosonic SPT phases. The symmetry twisted metrics play roles of nontrivial holonomy. Partition functions and correlation functions can be calculated in a unified framework. In particular, we showed that the partition functions on the torus, the real projective plane, and the Klein bottle match precisely with the SPT invariants constructed from the MPS method.

There is a number of natural extensions of the current paper: for example, it is natural to speculate that we can make a precise dictionary between higher-dimensional TFTs and higher-dimensional tensor networks, such as projected entangled pair states (PEPS). 
Another interesting direction is to consider topological phases of fermions, and their descriptions in terms of (fermionic) tensor networks, and spin TFTs. (For recent works addressing these issues, see refs. [32, 33] (the state sum construction of $(1+1) d$ oriented spin TFTs), refs. [34, 35] $((1+1) \mathrm{d}$ and $(2+1) d$ oriented equivariant spin TFTs and the state sum construction), and ref. [20].

Note added. After completing this work, we became aware of an independent work [36], which established the connection between the state sum construction of $(1+1) \mathrm{d} G$ equivariant TQFT and MPS representations.

\section{Acknowledgments}

We thank Gil Young Cho, Kiyonori Gomi, Andreas W. W. Ludwig, Kantaro Ohmori, Hassan Shapourian, Tadashi Takayanagi, Apoorv Tiwari, Keisuke Totsuka, Alex Turzillo, Juven C. Wang, Xueda Wen, and Peng Ye for useful discussion. Especially, K.S. is grateful to Takahiro Morimoto for pointing out the equivalence between a partial transposition and crosscap. This work was supported in part by the National Science Foundation grant DMR-1455296, and by Alfred P. Sloan foundation. K.S. is supported by JSPS Postdoctoral Fellowship for Research Abroad.

\section{A Group cohomology}

Let $G$ be a group and $\phi: G \rightarrow \mathbb{Z}_{2}$ be a homomorphism which specifies orientation preserving symmetries. Let $\mathrm{U}(1)_{\phi}$ be a $G$-left module defined by

$$
g \cdot z= \begin{cases}z & (\phi(g)=1) \\ z^{*} & (\phi(g)=-1)\end{cases}
$$

where $z \in \mathrm{U}(1)$. The cochain complex $C^{*}\left(G, \mathrm{U}(1)_{\phi}\right)$ is defined by the differential $\delta$ : $C^{n}\left(G, \mathrm{U}(1)_{\phi}\right) \rightarrow C^{n+1}\left(G, \mathrm{U}(1)_{\phi}\right)$ as

$$
\begin{aligned}
\delta c_{n}\left(g_{1}, \ldots g_{n+1}\right)= & c_{n}^{\phi\left(g_{1}\right)}\left(g_{2}, \ldots, g_{n+1}\right) c_{n}^{-1}\left(g_{1} g_{2}, g_{3}, \ldots, g_{n+1}\right) \cdots \\
& \cdots c_{n}\left(g_{1}, g_{2} g_{3}, g_{4}, \ldots, g_{n+1}\right) \cdots c_{n}^{(-1)^{n}}\left(g_{1}, \ldots, g_{n} g_{n+1}\right) c_{n}^{(-1)^{n+1}}\left(g_{1}, \ldots, g_{n}\right) .
\end{aligned}
$$

For our purposes, only the cases of $n=1,2$ are needed,

$$
\begin{aligned}
\delta c_{1}\left(g_{1}, g_{2}\right) & =\left(g_{1} \cdot c_{1}\right)\left(g_{2}\right) c_{1}^{-1}\left(g_{1} g_{2}\right) c_{1}\left(g_{1}\right)=c_{1}^{\phi\left(g_{1}\right)}\left(g_{2}\right) c_{1}^{-1}\left(g_{1} g_{2}\right) c_{1}\left(g_{1}\right), \\
\delta c_{2}\left(g_{1}, g_{2}, g_{3}\right) & =\left(g_{1} \cdot c_{2}\right)\left(g_{2}, g_{3}\right) c_{2}^{-1}\left(g_{1} g_{2}, g_{3}\right) c_{2}\left(g_{1}, g_{2} g_{3}\right) c_{2}^{-1}\left(g_{1}, g_{2}\right) \\
& =c_{2}^{\phi\left(g_{1}\right)}\left(g_{2}, g_{3}\right) c_{2}^{-1}\left(g_{1} g_{2}, g_{3}\right) c_{2}\left(g_{1}, g_{2} g_{3}\right) c_{2}^{-1}\left(g_{1}, g_{2}\right),
\end{aligned}
$$

where $c_{n}: G^{n} \rightarrow \mathrm{U}(1)$. The group cohomology (with $\mathrm{U}(1)_{\phi}$ coefficient) is defined by

$$
H^{n}\left(G, \mathrm{U}(1)_{\phi}\right)=Z^{2}\left(G, \mathrm{U}(1)_{\phi}\right) / B^{n}\left(G, \mathrm{U}(1)_{\phi}\right) .
$$

For a trivial $G$-module $\mathrm{U}(1)$, we have

$$
H^{n}(G, \mathrm{U}(1)) \cong H^{n+1}(G, \mathbb{Z}) .
$$




\section{B Projective representation}

Once a 2-group cocycle $b(g, h) \in Z^{2}(G, \mathrm{U}(1))$ is given, a factor group of a $b$-projective representation is determined as

$$
V_{g} V_{h}=b(g, h) V_{g h}
$$

In the same manner as the ordinary linear representation, there may be multiple irreducible $b$-projective representations. The following quantity

$$
\sum_{V \in b \text {-irreps. }}(\operatorname{dim} V)^{2}=\text { const }
$$

does not depend on $b(g, h) \in Z^{2}(G, \mathrm{U}(1))$. For example, for $G=\mathbb{Z}_{n} \times \mathbb{Z}_{n}=$ $\left\langle\sigma_{1}, \sigma_{2} \mid \sigma_{1}^{n}=\sigma_{2}^{n}=1\right\rangle$, the second group cohomology is $H^{2}\left(\mathbb{Z}_{n} \times \mathbb{Z}_{n}, \mathrm{U}(1)\right)=\mathbb{Z}_{n}$, and there are $n^{2} 1$-dimensional linear irreps. and only one $n$-dimensional nontrivial projective irreps., thus, the above identity holds as

$$
\underbrace{1^{2}+\cdots+1^{2}}_{n^{2}}=n^{2}
$$

The trivial linear irreps. are constructed as

$$
V_{\sigma_{1}}=e^{2 p \pi i / n}, \quad V_{\sigma_{2}}=e^{2 q \pi i / n}, \quad(p, q=0, \ldots, n-1) .
$$

On the other hand, a nontrivial projective irreps. belonging to $1 \in H^{2}\left(\mathbb{Z}_{n} \times \mathbb{Z}_{n}, \mathrm{U}(1)\right)$ is given by

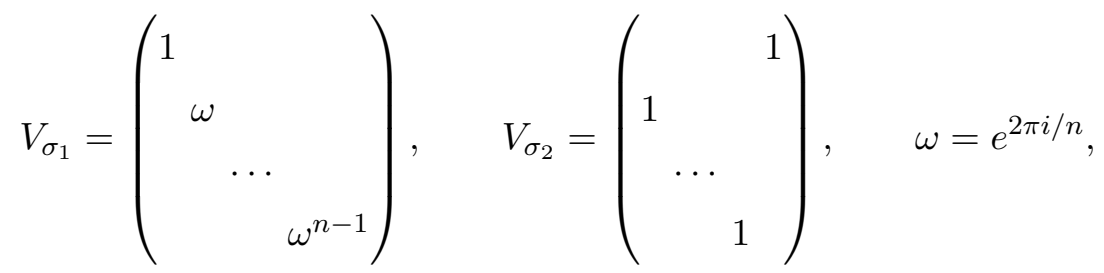

which satisfies $V_{\sigma_{1}} V_{\sigma_{2}}=\omega V_{\sigma_{2}} V_{\sigma_{1}}$. In general, for commuting elements $g, h \in G$, we have

$$
V_{g} V_{h}=\epsilon(g, h) V_{h} V_{g}, \quad(g h=h g) .
$$

An example of the existence of multiple projective irreps. is a dihedral group $D_{4}=$ $\left\langle c_{4}, \sigma \mid c_{4}^{4}=\sigma^{2}=1, \sigma c_{4} \sigma=c_{4}^{-1}\right\rangle$ of which the second group cohomology is $H^{2}\left(D_{4}, \mathrm{U}(1)\right)=$ $\mathbb{Z}_{2}$. For $1 \in H^{2}\left(D_{4}, \mathrm{U}(1)\right)$, there are two inequivalent irreps. $V_{g}, W_{g}$ as

$$
\left\{\begin{array} { l } 
{ V _ { c _ { 4 } } = e ^ { - \frac { \pi } { 4 } i \sigma _ { y } } } \\
{ V _ { \sigma } = \sigma _ { z } }
\end{array} \quad \left\{\begin{array}{l}
W_{c_{4}}=e^{-\frac{3 \pi}{4} i \sigma_{y}} \\
W_{\sigma}=\sigma_{z}
\end{array}\right.\right.
$$

Here $\sigma_{i}(i=x, y, z)$ are the Pauli matrices. 


\section{Orbifolding: Dijkgraaf-Witten theory in $(1+1) d$}

In section 2.4, we have discussed gauging of symmetry $G$. The partition function $Z_{M}(P)$ on a 2 -space $M$ with various background $G$-field $P$ gives the topological invariants for $(1+1) \mathrm{d}$ bosonic SPT phase. In other words, what we have discussed are the response theory of SPT phases. One can make one further step and consider orbifolding by summing over all possible flat background $G$-field. (I.e., we are promoting the (flat) $G$-field to dynamical entities.) This procedure leads to the so-called the Dijkgraaf-Witten theory [11, 37-40] in $(1+1)$ d. Here we show the partition functions of the orbifolded theories on some spacetimes:

$$
\begin{aligned}
Z_{\text {orb }}\left(T^{2}\right) & =\frac{1}{|G|} \sum_{g, h \in G_{0} ;[g, h]=0} \epsilon(g, h)=\text { number of } b \text {-irreps., } \\
Z_{\text {orb }}(K B) & =\frac{1}{|G|} \sum_{g \notin G_{0}, h \in G_{0} ; g h^{-1} g^{-1}=h} \kappa(g ; h), \\
Z_{\text {orb }}\left(\mathbb{R} P^{2}\right) & =\frac{1}{|G|} \sum_{g \notin G_{0} ; g^{2}=1} \theta(g) .
\end{aligned}
$$

\section{Algebraic relations in equivariant open and closed TFTs}

In this section, we summarize the algebraic relations which are followed from the $G$ equivariant cobordism category. [10-12, 29, 30] In the following picture, dotted lines without specifying a group element represent trivial holonomies.

\section{D.1 Closed TFT}

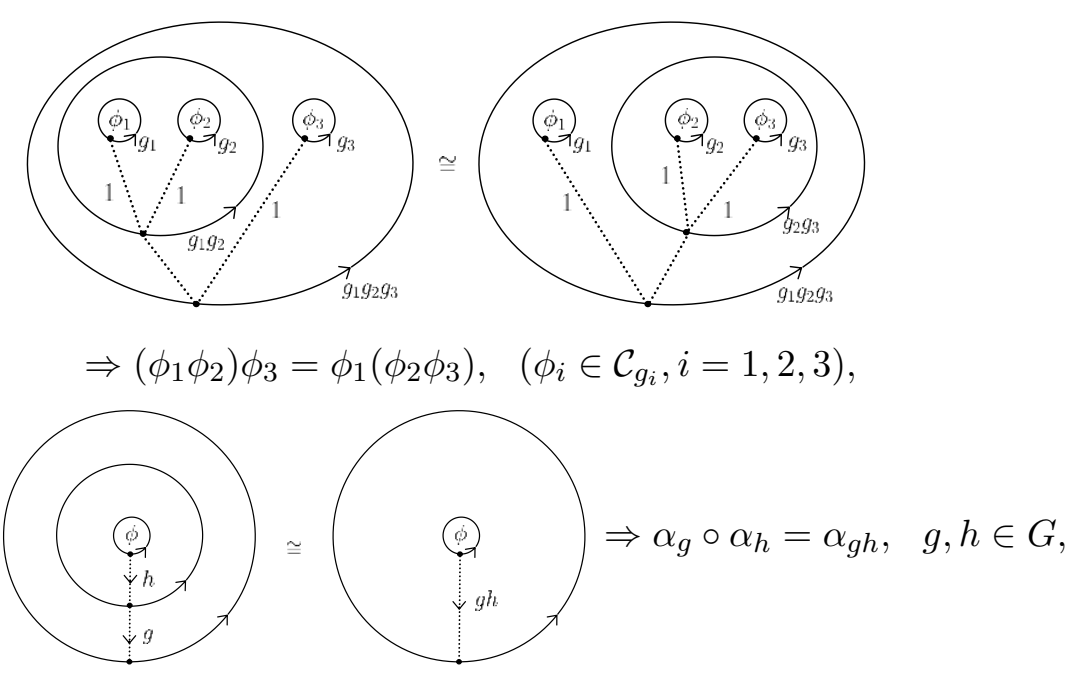




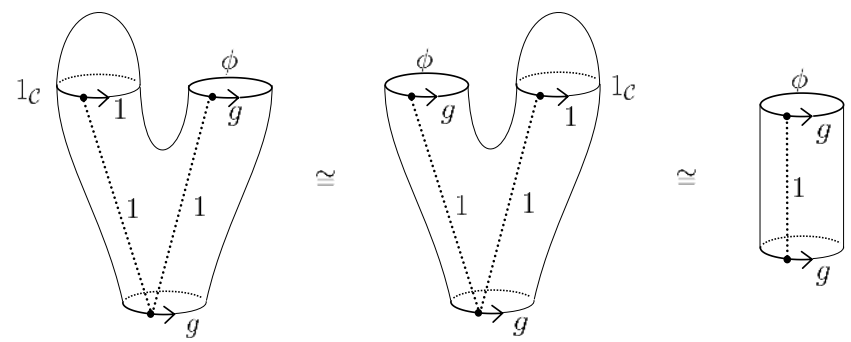

$$
\Rightarrow 1_{\mathcal{C}} \phi=\phi 1_{\mathcal{C}}=\phi, \quad\left(\phi \in \mathcal{C}_{g}\right),
$$

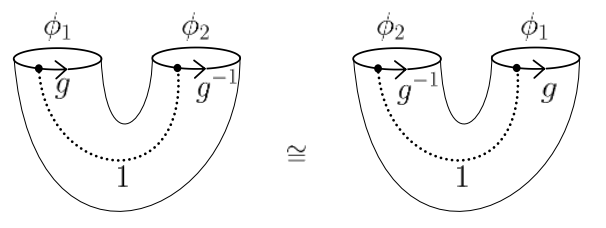

$\Rightarrow \theta_{\mathcal{C}}\left(\phi_{1} \phi_{2}\right)=\theta_{\mathcal{C}}\left(\phi_{2} \phi_{1}\right), \quad\left(\phi_{1} \in \mathcal{C}_{g}, \phi_{2} \in \mathcal{C}_{g^{-1}}\right)$,
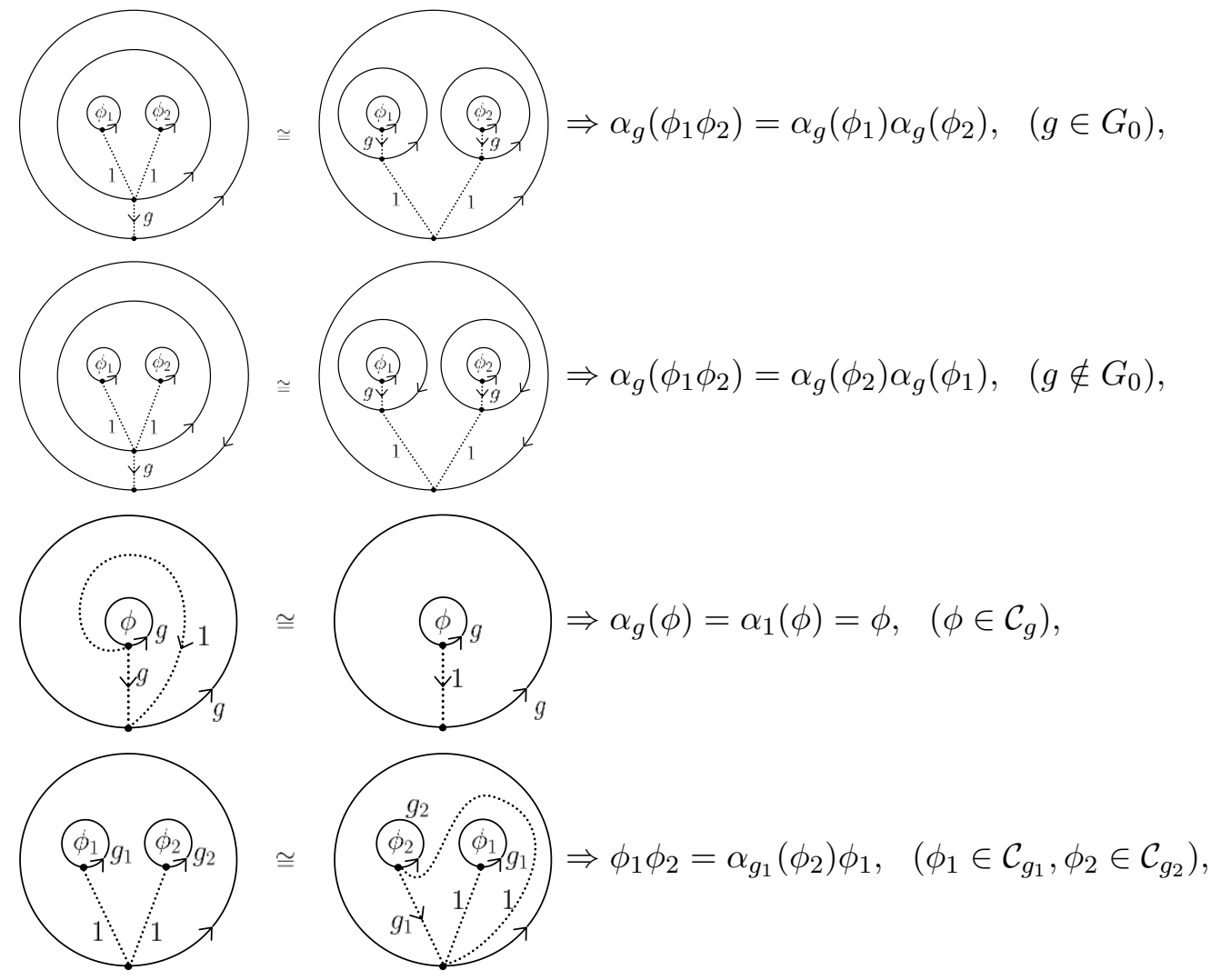

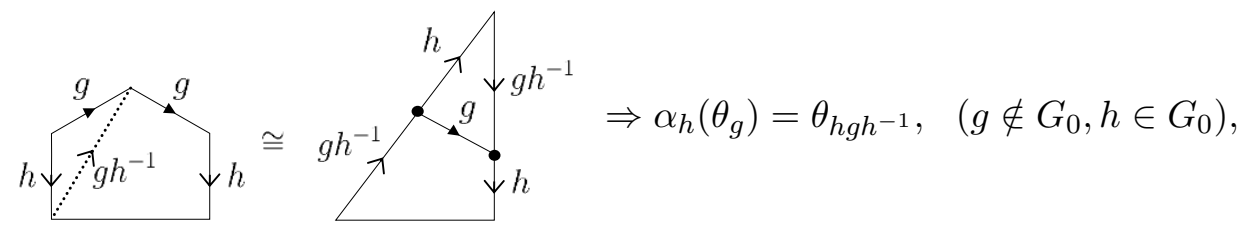



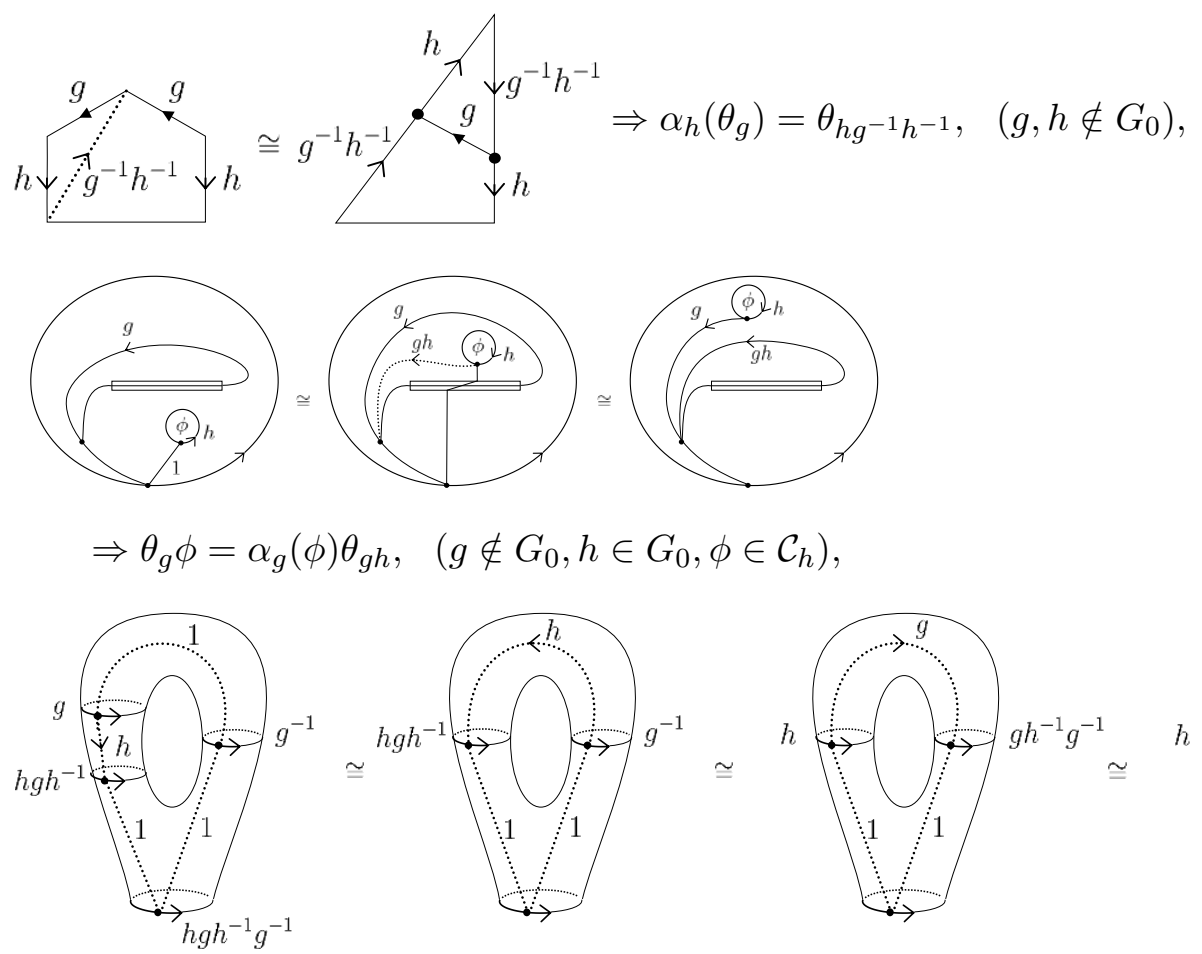

$$
\Rightarrow \sum_{i} \alpha_{h}\left(\xi_{i}^{g}\right) \xi_{i}^{g^{-1}}=\sum_{i} \xi_{i}^{h} \alpha_{g}\left(\xi_{i}^{h^{-1}}\right),
$$
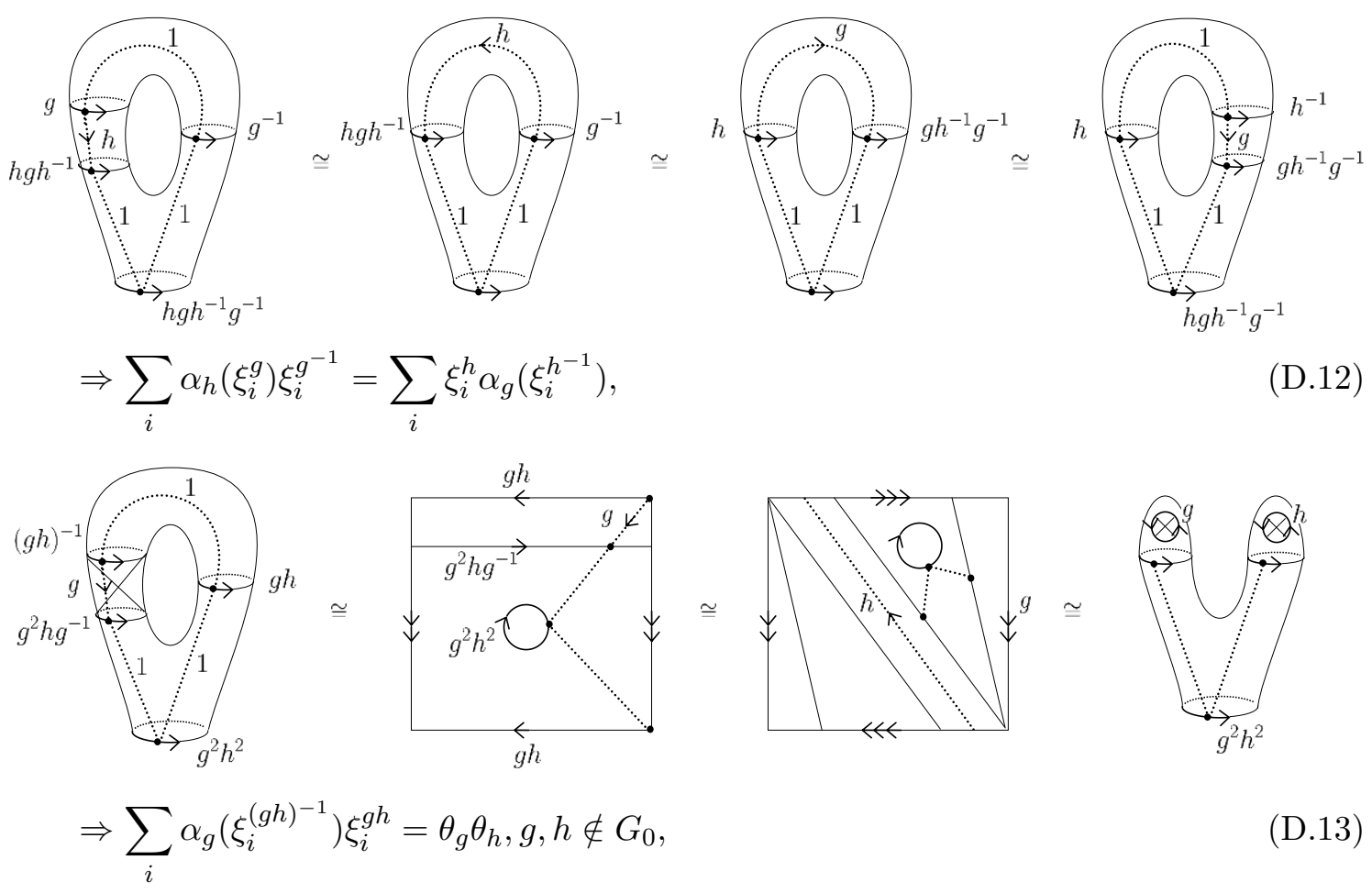

\section{D.2 Open TFT}

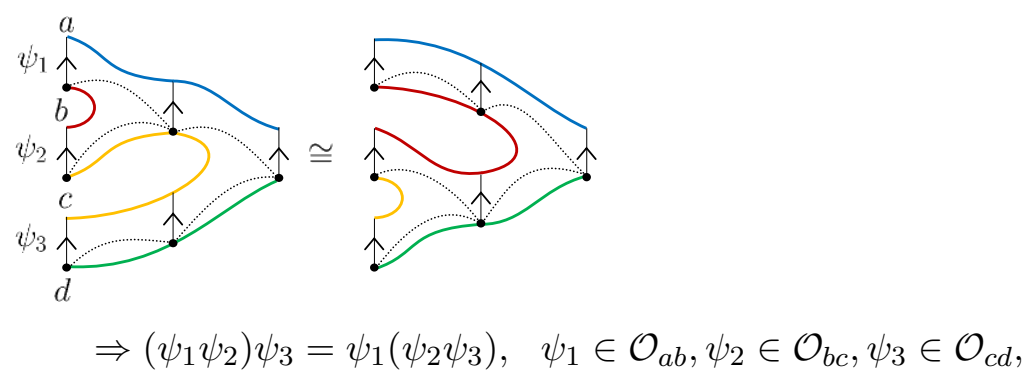



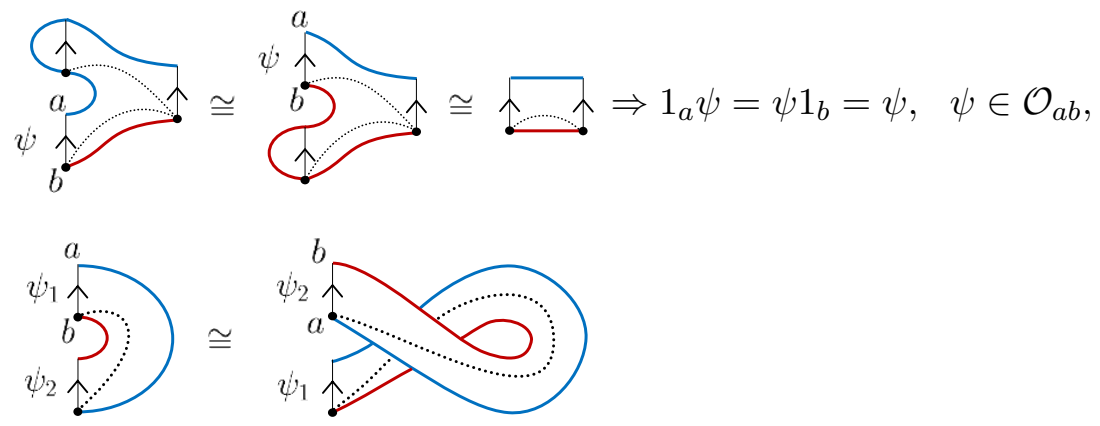

$\Rightarrow \theta_{a}\left(\psi_{1} \psi_{2}\right)=\theta_{b}\left(\psi_{2} \psi_{1}\right), \quad \psi_{1} \in \mathcal{O}_{a b}, \psi_{2} \in \mathcal{O}_{b a}$,
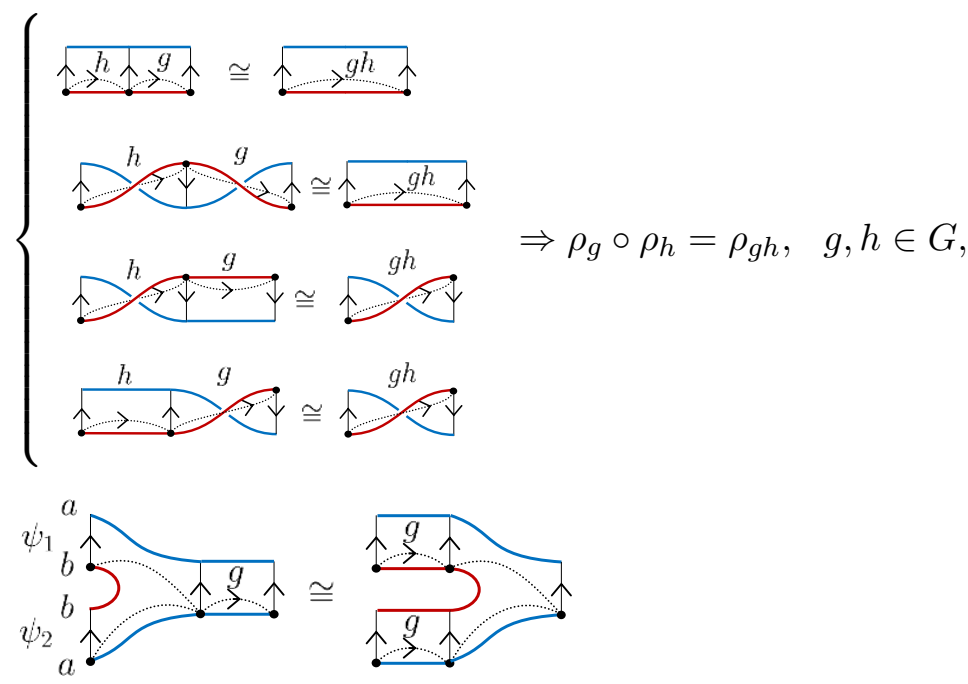

$\Rightarrow \rho_{g \in G_{0}}\left(\psi_{1} \psi_{2}\right)=\rho_{g}\left(\psi_{1}\right) \rho_{g}\left(\psi_{2}\right), \quad \psi_{1} \in \mathcal{O}_{a b}, \psi_{2} \in \mathcal{O}_{b a}$,

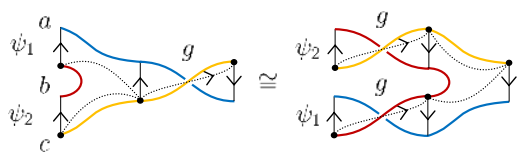

$\Rightarrow \rho_{g \in G_{0}}\left(\psi_{1} \psi_{2}\right)=\rho_{g}\left(\psi_{2}\right) \rho_{g}\left(\psi_{1}\right), \quad \psi_{1} \in \mathcal{O}_{a b}, \psi_{2} \in \mathcal{O}_{b a}$,

$\checkmark \hat{\imath}$
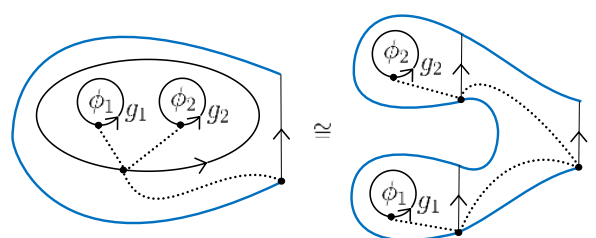

$\Rightarrow \imath_{g_{1} g_{2}, a}\left(\phi_{1} \phi_{2}\right)=\imath_{g_{2}, a}\left(\phi_{2}\right) \imath_{g_{1}, a}\left(\phi_{1}\right), \quad \phi_{1} \in \mathcal{C}_{g_{1}}, \phi_{2} \in \mathcal{C}_{g_{2}}$, 

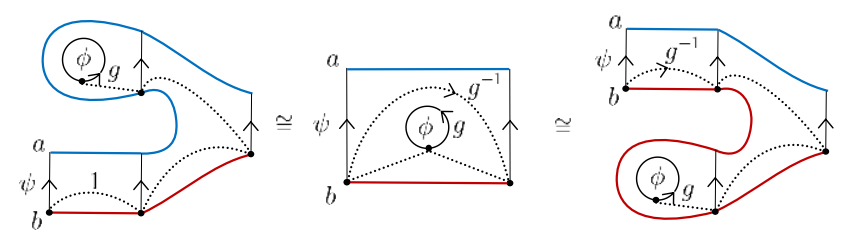

$$
\Rightarrow \imath_{g, a}(\phi) \psi=\rho_{g^{-1}}(\psi) \imath_{g, b}(\phi), \quad \phi \in \mathcal{C}_{g}, \psi \in \mathcal{O}_{a b}
$$
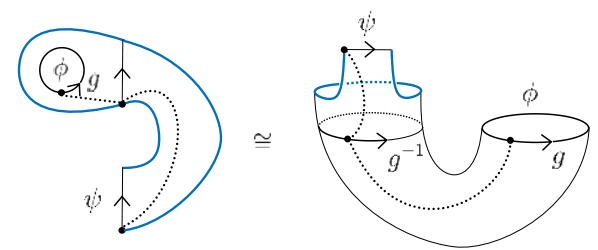

$$
\Rightarrow \theta_{a}\left(\imath_{g, a}(\phi) \psi\right)=\theta_{\mathcal{C}}\left(\imath^{g^{-1}, a}(\psi) \phi\right), \quad \phi \in \mathcal{C}_{g}, \psi \in \mathcal{O}_{a a}
$$
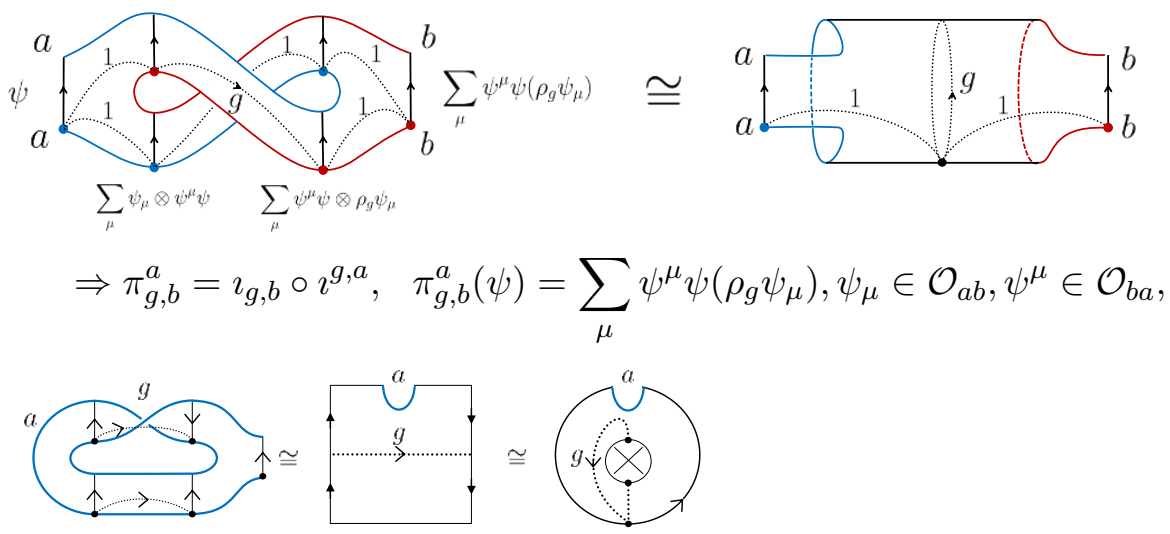

$$
\Rightarrow \sum_{\mu}\left(\rho_{g} \psi_{\mu}\right) \psi^{\mu}=\imath_{g^{2}, a}\left(\theta_{g}\right), \quad g \notin G_{0} .
$$

Open Access. This article is distributed under the terms of the Creative Commons Attribution License (CC-BY 4.0), which permits any use, distribution and reproduction in any medium, provided the original author(s) and source are credited.

\section{References}

[1] X. Chen, Z.-C. Gu, Z.-X. Liu and X.-G. Wen, Symmetry protected topological orders and the group cohomology of their symmetry group, Phys. Rev. B 87 (2013) 155114 [arXiv:1106.4772] [INSPIRE].

[2] X. Chen, Z.-C. Gu and X.-G. Wen, Classification of gapped symmetric phases in one-dimensional spin systems, Phys. Rev. B 83 (2011) 035107 [arXiv:1008.3745].

[3] N. Schuch, D. Pérez-García and I. Cirac, Classifying quantum phases using matrix product states and projected entangled pair states, Phys. Rev. B 84 (2011) 165139 [arXiv: 1010.3732].

[4] F. Pollmann, E. Berg, A.M. Turner and M. Oshikawa, Symmetry protection of topological phases in one-dimensional quantum spin systems, Phys. Rev. B 85 (2012) 075125 [arXiv:0909.4059]. 
[5] F. Pollmann and A.M. Turner, Detection of symmetry-protected topological phases in one dimension, Phys. Rev. B 86 (2012) 125441 [arXiv: 1204.0704].

[6] X.-G. Wen, Quantum field theory of many-body systems: from the origin of sound to an origin of light and electrons, Oxford University Press, Oxford U.K., (2004) [INSPIRE].

[7] E. Fradkin, Field theories of condensed matter physics, Cambridge University Press, Cambridge U.K., (2013) [INSPIRE].

[8] V. Turaev, Homotopy field theory in dimension two and group algebras, math/9910010 [INSPIRE].

[9] V.G. Turaev, Homotopy quantum field theory, vol. 10, European Mathematical Society, (2010).

[10] G.W. Moore, Lectures on branes, $k$-theory and rr charges; two variations on the theme of $2 D$ TFT, Clay mathematical institute lectures, http://www.physics.rutgers.edu/ gmoore/clay.html, U.S.A., (2002).

[11] G.W. Moore and G. Segal, D-branes and k-theory in $2 D$ topological field theory, hep-th/0609042 [INSPIRE].

[12] A. Kapustin and A. Turzillo, Equivariant topological quantum field theory and symmetry protected topological phases, JHEP 03 (2017) 006 [arXiv: 1504.01830] [INSPIRE].

[13] C.-I. Lazaroiu, On the structure of open-closed topological field theory in two-dimensions, Nucl. Phys. B 603 (2001) 497 [hep-th/0010269] [INSPIRE].

[14] M. Fukuma, S. Hosono and H. Kawai, Lattice topological field theory in two-dimensions, Commun. Math. Phys. 161 (1994) 157 [hep-th/9212154] [INSPIRE].

[15] V. Karimipour and A. Mostafazadeh, Lattice topological field theory on nonorientable surfaces, J. Math. Phys. 38 (1997) 49 [hep-th/9508041] [INSPIRE].

[16] D. Pérez-García, M.M. Wolf, M. Sanz, F. Verstraete and J.I. Cirac, String order and symmetries in quantum spin lattices, Phys. Rev. Lett. 100 (2008) 167202 [arXiv:0802. 0447].

[17] L. Fidkowski and A. Kitaev, Topological phases of fermions in one dimension, Phys. Rev. B 83 (2011) 075103 [arXiv: 1008.4138].

[18] I. Affleck, T. Kennedy, E.H. Lieb and H. Tasaki, Valence bond ground states in isotropic quantum antiferromagnets, in Condensed matter physics and exactly soluble models, Springer, Berlin Germany, (1988), pg. 253 [Commun. Math. Phys. 115 (1988) 477] [inSPIRE].

[19] X. Chen, Z.-C. Gu, Z.-X. Liu and X.-G. Wen, Symmetry-protected topological orders in interacting bosonic systems, Science 338 (2012) 1604 [arXiv:1301.0861].

[20] H. Shapourian, K. Shiozaki and S. Ryu, Detection of symmetry-protected topological phases in fermionic many-body systems, arXiv:1607.03896.

[21] K. Shiozaki, H. Shapourian and S. Ryu, Many-body topological invariants in fermionic symmetry protected topological phases, arXiv:1609.05970 [INSPIRE].

[22] J. Haegeman, D. Pérez-García, I. Cirac and N. Schuch, Order parameter for symmetry-protected phases in one dimension, Phys. Rev. Lett. 109 (2012) 050402.

[23] X.-L. Qi, H. Katsura and A.W.W. Ludwig, General relationship between the entanglement spectrum and the edge state spectrum of topological quantum states, Phys. Rev. Lett. 108 (2012) 196402 [arXiv:1103.5437]. 
[24] G. Vidal and R.F. Werner, Computable measure of entanglement, Phys. Rev. A 65 (2002) 032314 [quant-ph/0102117].

[25] M. Atiyah, Topological quantum field theories, Inst. Hautes Études Sci. Publ. Math. 68 (1989) 175 [INSPIRE].

[26] F. Quinn, Lectures on axiomatic topological quantum field theory, in Geometry and quantum field theory, D. Freed and K. Uhlenbeck eds., IAS/Park City Math. Ser. 1, Amer. Math. Soc., U.S.A., (1995) [INSPIRE].

[27] X. Chen, Z.-C. Gu and X.-G. Wen, Complete classification of one-dimensional gapped quantum phases in interacting spin systems, Phys. Rev. B 84 (2011) 235128 [arXiv:1103.3323].

[28] V. Turaev and P. Turner, Link homology and unoriented topological quantum field theory, Alg. Geom. Topol. 6 (2006) 1069 [math/0506229] [INSPIRE].

[29] K. Tagami, Unoriented HQFTs and its underlying algebras, Topol. Appl. 159 (2012) 833 [arXiv:1104.2372] [INSPIRE].

[30] R. Sweet, Equivariant unoriented topological field theories and G-extended Frobenius algebras, Ph.D. thesis, Boston University, Boston U.S.A., (2013).

[31] G.Y. Cho, K. Shiozaki, S. Ryu and A.W.W. Ludwig, Relationship between symmetry protected topological phases and boundary conformal field theories via the entanglement spectrum, arXiv:1606.06402 [INSPIRE].

[32] J.W. Barrett and S.O.G. Tavares, Two-dimensional state sum models and spin structures, Commun. Math. Phys. 336 (2015) 63 [arXiv:1312.7561] [INSPIRE].

[33] S. Novak and I. Runkel, State sum construction of two-dimensional topological quantum field theories on spin surfaces, J. Knot Theor. Ramifications 24 (2015) 1550028 [arXiv: 1402.2839] [INSPIRE].

[34] D. Gaiotto and A. Kapustin, Spin TQFTs and fermionic phases of matter, Int. J. Mod. Phys. A 31 (2016) 1645044 [arXiv: 1505.05856] [INSPIRE].

[35] L. Bhardwaj, D. Gaiotto and A. Kapustin, State sum constructions of spin-TFTs and string net constructions of fermionic phases of matter, arXiv: 1605.01640 [INSPIRE].

[36] A. Kapustin, A. Turzillo and M. You, Topological field theory and matrix product states, arXiv: 1607.06766 [INSPIRE].

[37] R. Dijkgraaf and E. Witten, Topological gauge theories and group cohomology, Commun. Math. Phys. 129 (1990) 393 [InSPIRE].

[38] M. Levin and Z.-C. Gu, Braiding statistics approach to symmetry-protected topological phases, Phys. Rev. B 86 (2012) 115109 [arXiv:1202.3120] [INSPIRE].

[39] S. Ryu and S.-C. Zhang, Interacting topological phases and modular invariance, Phys. Rev. B 85 (2012) 245132 [arXiv: 1202.4484] [INSPIRE].

[40] O.M. Sule, X. Chen and S. Ryu, Symmetry-protected topological phases and orbifolds: generalized Laughlin's argument, Phys. Rev. B 88 (2013) 075125 [arXiv:1305.0700] [INSPIRE]. 